\title{
F-theory, GUTs, and the Weak Scale
}

\section{Citation}

Heckman, Jonathan, and Cumrun Vafa. 2009. F-theory, GUTs, and the Weak Scale. Journal of High Energy Physics 079.

\section{Published Version}

doi://10.1088/1126-6708/2009/09/079

\section{Permanent link}

http://nrs.harvard.edu/urn-3:HUL.InstRepos:42656557

\section{Terms of Use}

This article was downloaded from Harvard University's DASH repository, and is made available under the terms and conditions applicable to Open Access Policy Articles, as set forth at http:// nrs.harvard.edu/urn-3:HUL.InstRepos:dash.current.terms-of-use\#OAP

\section{Share Your Story}

The Harvard community has made this article openly available.

Please share how this access benefits you. Submit a story.

\section{Accessibility}




\title{
F-theory, GUTs, and the Weak Scale
}

\author{
Jonathan J. Heckman* and Cumrun Vafa ${ }^{\dagger}$ \\ Jefferson Physical Laboratory, Harvard University, Cambridge, MA 02138, USA
}

\begin{abstract}
In this paper we study a deformation of gauge mediated supersymmetry breaking in a class of local F-theory GUT models where the scale of supersymmetry breaking determines the value of the $\mu$ term. Geometrically correlating these two scales constrains the soft SUSY breaking parameters of the MSSM. In this scenario, the hidden SUSY breaking sector involves an anomalous $U(1)$ Peccei-Quinn symmetry which forbids bare $\mu$ and $B \mu$ terms. This sector typically breaks supersymmetry at the desired range of energy scales through a simple stringy hybrid of a Fayet and Polonyi model. A variant of the Giudice-Masiero mechanism generates the value $\mu \sim 10^{2}-10^{3} \mathrm{GeV}$ when the hidden sector scale of supersymmetry breaking is $\sqrt{F} \sim 10^{8.5} \mathrm{GeV}$. Further, the $B \mu$ problem is solved due to the mild hierarchy between the GUT scale and Planck scale. These models relate SUSY breaking with the QCD axion, and solve the strong CP problem through an axion with decay constant $f_{a} \sim M_{G U T} \cdot \mu / \Lambda$, where $\Lambda \sim 10^{5} \mathrm{GeV}$ is the characteristic scale of gaugino mass unification in gauge mediated models, and the ratio $\mu / \Lambda \sim M_{G U T} / M_{p l} \sim 10^{-3}$. We find $f_{a} \sim 10^{12} \mathrm{GeV}$, which is near the high end of the phenomenologically viable window. Here, the axino is the goldstino mode which is eaten by the gravitino. The gravitino is the LSP with a mass of about $10^{1}-10^{2} \mathrm{MeV}$, and a bino-like neutralino is (typically) the NLSP with mass of about $10^{2}-10^{3} \mathrm{GeV}$. Compatibility with electroweak symmetry breaking also determines the value of $\tan \beta \sim 30 \pm 7$.
\end{abstract}

September, 2008

\footnotetext{
*e-mail: jheckman@fas.harvard.edu
}

${ }^{\dagger}$ e-mail: vafa@physics.harvard.edu 


\section{Contents}

1 Introduction

2 Review of Minimal F-theory GUTs 10

2.1 Supersymmetry Breaking and Local Models . . . . . . . . . . . . . 12

3 Review of Gauge Mediation and the $\mu / B \mu$ Problem 12

3.1 Interpolating from Gravity/Moduli Mediation to Gauge Mediation . . 15

4 Variants of the Giudice-Masiero Mechanism and Higher Dimension Operators

4.1 Giudice-Masiero Operators . . . . . . . . . . . . . . . 22

$4.2 U(1)_{P Q}$ Induced Soft Mass Terms . . . . . . . . . . . . . 26

5 Diamond Ring Model 27

6 Strong CP and Gauge Mediation 31

$7 E_{6}, U(1)_{P Q}$ and F-theory

8 Fayet-Polonyi Model of Supersymmetry Breaking 47

8.1 Generalities of Fayet-Polonyi Model . . . . . . . . . . . . . 48

8.2 Fayet-Polonyi Model from a $U(1)_{P Q}$ Seven-Brane . . . . . . . . . . . 50

8.2.1 D-Term Potential . . . . . . . . . . . . . 52

8.2 .2 F-Term Potential . . . . . . . . . . . . . 54

8.3 Fayet-Polonyi Model . . . . . . . . . . . . . . . . . 56

8.4 Stabilizing the Radial Mode . . . . . . . . . . . . . . . . . 59

9 Region of MSSM Parameter Space 65

9.1 UV Boundary Conditions . . . . . . . . . . . . . . . 67

9.2 Constraining the MSSM . . . . . . . . . . . . . . . 68 
A Higher Order Instanton Corrections and the Axion Potential 81

A.1 Constraints From Symmetries . . . . . . . . . . . . 81

A.2 Estimates on Higher Order Corrections . . . . . . . . . . . . . . 82

A.3 Achieving a Flat Axion Potential . . . . . . . . . . 85 


\section{Introduction}

The existence of the string theory landscape with its vast number of different low energy signatures potentially dilutes the predictive power of string theory. The sheer range of possibilities presents a challenge to determine which corners of the landscape (if any!) are consistent with experiment.

Decoupling the dynamics of gauge theory from gravity provides an attractive way to constrain this problem. Indeed, at energy scales even a few orders of magnitude below the Planck scale, gravity will most likely play a subdominant role compared to other degrees of freedom. For considerations at lower energies it is therefore quite natural to restrict attention to vacua in the landscape with the correct gauge and matter content, deferring all issues pertaining to gravity to a later stage of analysis. Given the vast size of the landscape, it is also reasonable to incorporate some additional principles in our search for vacua which are consistent with observation.

One such constraint is the apparent unification of the gauge coupling constants of the MSSM at an energy below the Planck scale. Here, it is important to note that the existence of a Grand Unified Theory (GUT) is in principle compatible with the existence of a decoupling limit. For example, in minimal realizations of GUTs, the gauge theory is typically asymptotically free. Indeed, without asymptotic freedom, the UV completion of the gauge theory would require incorporating gravity into the theory. Moreover, while string theory can accommodate the generic representations of GUTs, the total matter content in such models often does not produce an asymptotically free theory. In this regard, the existence of a decoupling limit is especially helpful in limiting any search for realistic models.

In string compactifications, the requirement that gravity can in principle decouple translates into the geometric condition that some of the dimensions of the compactification can in principle expand to a large size while the GUT model degrees of freedom remain localized on a compact cycle. Roughly speaking, the decoupling principle separates the "open string" and "closed string" sectors of the landscape.

In $[1,2]$, we initiated a study of F-theory GUTs which admit such a decoupling limit. These local F-theory models provide a surprisingly rigid framework for model building [2]. See see also [3-7] for related work on model building in F-theory. In F-theory, local GUT models originate from a stack of seven-branes wrapping a del Pezzo surface equipped with gauge group $S U(5)$, or some larger rank GUT group 
which contains $S U(5)$. Although the matter content of these models can in principle originate from either bulk eight-dimensional fields propagating on the seven-brane, or six-dimensional fields localized at the intersection of distinct stacks of seven-branes, in minimal $S U(5)$ GUT models, all of the chiral matter of the MSSM localizes at the intersection of seven-branes along Riemann surfaces in the del Pezzo surface. The GUT group breaks to the Standard Model gauge group in the presence of an internal $U(1)$ hypercharge flux through the del Pezzo surface.

As shown in [2], simply achieving the correct matter content of the MSSM through an appropriate choice of internal fluxes turns out to simultaneously address several puzzles in four-dimensional GUT models. For example, solving the doublet-triplet splitting problem via an internal $U(1)$ hyperflux required for GUT group breaking automatically forbids quartic superpotential terms which can cause rapid proton decay. The presence of this same hyperflux can also qualitatively explain why the lighter two generations violate the analogue of the well-known $b-\tau$ mass relation. Precisely because this framework is so rigid, it is also possible to reliably extract predictions for the neutrino masses which are fortuitiously in accord with current experimental bounds.

To make further contact with observation, any viable model must incorporate a sector which breaks supersymmetry, and a mediation mechanism which communicates this to the visible sector. From the perspective of the four-dimensional effective field theory, this mediation mechanism is likely to originate from gravity/moduli mediation, gauge mediation, or some variant of these two basic possibilities 1 Both mediation mechanisms contain problems, either with correlating the scale of supersymmetry breaking with the superpotential contribution to the Higgs mass $\mu H_{u} H_{d}$ known as the $\mu$ term, or from large flavor changing neutral currents (FCNCs). In gravity/moduli mediation scenarios, the $\mu$ problem can be solved via the GiudiceMasiero mechanism [14], but the generic pattern of soft masses will often generate large FCNCs, which is typically not a problem in models of gauge mediated supersymmetry breaking. Nevertheless, gravity/moduli mediation has recently been in-

\footnotetext{
${ }^{1}$ It is also possible to consider other scenarios such as anomaly mediation $[8,9]$ where the scale of supersymmetry breaking in the hidden sector is typically higher than in gravity/moduli mediation. As another somewhat related possibility, it is also possible to consider $U(1)$ mediation models as in $[10,11]$ and its recent implementation in a compact string based model with F- and D- term mixing [12]. To mention just one further possibility, D-brane instantons could perhaps provide a more stringy mediation mechanism [13].
} 
vestigated in F-theory models as in the recent attractive proposal of [5]. See [15-18] and especially [19] for further details on moduli mediation scenarios in type IIB compactifications.

By contrast, the primary stumbling block to realistic phenomenology in most models of gauge mediation is the $\mu / B \mu$ problem. Indeed, perturbative gauge interactions do not generate superpotential terms involving the Higgs fields. Precisely because gauge fields mediate the effects of supersymmetry breaking to the visible sector, correlating the size of the $\mu$ term with supersymmetry breaking remains somewhat obscure in such a scenario. Logically speaking, however, the values of $\mu$ and $B \mu$ may have nothing to do with supersymmetry breaking. For example, in [2], exponential wave function suppression near the GUT model seven-brane could also generate small values for the $\mu$ term. Although this relieves much of the tension present in gauge mediated scenarios, it is also not very predictive! In this paper, we shall therefore not consider this possibility further.

A central goal of the present paper is to address the $\mu / B \mu$ problem in variants of gauge mediated supersymmetry breaking which can arise in F-theory. Focussing on gauge mediation is particularly reasonable in the context of decoupling the "open" and "closed" string sectors of the landscape. However, as explained above, correlating the origin of $\mu / B \mu$ with supersymmetry breaking requires some deformation away from a minimal realization of the gauge mediation scenario. In fact, we find that solving the $\mu$ problem in F-theory sometimes requires that the mediation mechanism can decouple from gravity.

Regardless of the mediation mechanism, one natural way to correlate the value of $\mu$ with the scale of supersymmetry breaking is through variants of the GiudiceMasiero mechanism. In this solution to the $\mu$ problem, the Higgs chiral superfields $H_{u}$ and $H_{d}$ couple to a GUT group singlet $X$ through an interaction term of the form:

$$
O_{X^{\dagger} H_{u} H_{d}}=\gamma \int d^{4} \theta \frac{X^{\dagger} H_{u} H_{d}}{M_{X}},
$$

where $\gamma$ is an order one constant which depends on the details of the model, and $M_{X}$ is roughly the scale at which this operator is generated. When $X$ develops a supersymmetry breaking vev $\langle X\rangle=x+\theta^{2} F$, the resulting contribution to the $\mu$ term is $\gamma \cdot \bar{F} / M_{X}$. In gravity-mediated scenarios, $F \sim 10^{21}-10^{22} \mathrm{GeV}^{2}$, and $M_{X}$ is identified with the Planck scale. For lower values of $F \leq 10^{19} \mathrm{GeV}^{2}$ consistent with 
gauge mediation, the induced value of $\mu$ is near the weak scale provided $M_{X}$ is near or below the GUT scale. This curious numerology has been observed in [20] and has recently formed the basis for the "sweet spot" model of supersymmetry breaking [21] (also see [22]). See [6] for a recent effort in replicating the effective field theory of the sweet spot scenario in the context of a local F-theory model.

One of the primary results of this paper is that integrating out the Kaluza-Klein modes associated with $X$ generates the operator $O_{X^{\dagger} H_{u} H_{d}}$ with $M_{X}$ near the GUT scale. To a certain extent, this is to be expected because if this operator is present in a local model where gravity can in principle decouple, the scale of suppression will be set by the GUT, rather than Planck scale. As a consequence, the value of $F$ required in gravity mediated scenarios will generate a value for the $\mu$ term which is far too large. Hence, crude considerations already reveal that in a broad class of models, some variant of gauge mediation must transmit supersymmetry breaking to the MSSM. As in most perturbatively realized gauge mediated models, this implies that $X$ must also couple to at least one vector-like pair of messenger fields $Y$ and $Y^{\prime}$ through the F-term:

$$
O_{X Y Y^{\prime}}=\lambda \int d^{2} \theta X Y Y^{\prime} .
$$

Although different from the models we present, for recent related work on gauge mediated models of supersymmetry breaking in string theory, see [6,23-25].

Of course, in order for this variant on the Giudice-Masiero mechanism to solve the $\mu$ problem, additional unwanted contributions to this term must also be absent. For example, to prevent the presence of a large bare $\mu$ term, it is natural for all fields to transform under a $U(1)$ Peccei-Quinn (PQ) symmetry with charges:

\begin{tabular}{|l|r|r|r|r|}
\hline & $\Phi$ & $H_{u}, H_{d}$ & $Y, Y^{\prime}$ & $X$ \\
\hline$U(1)_{P Q}$ & +1 & -2 & +2 & -4 \\
\hline
\end{tabular}

where in the above table, $\Phi$ denotes any chiral superfield of the MSSM other than the Higgs up/down. This symmetry allows all requisite interaction terms of the MSSM as well as the operators $O_{X^{\dagger} H_{u} H_{d}}$ and $O_{X Y Y^{\prime}}$, but forbids the typically problematic superpotential terms such as a bare $\mu$ term $\mu H_{u} H_{d}$, as well as $X H_{u} H_{d}$. As explained in [2], the existence of such $U(1)$ symmetries is quite common in F-theory compactifications because matter fields originate from the intersection of distinct stacks of 
seven-branes.

We also find that the presence of the $U(1)_{P Q}$ symmetry generates additional soft term contributions beyond those present in a gauge mediated model. This $U(1)_{P Q}$ symmetry is typically anomalous and is therefore Higgsed at high energies. In this case, heavy $U(1)_{P Q}$ gauge boson exchange between $X$ and a generic chiral superfield $\Psi$ charged under $U(1)_{P Q}$ generates the term:

$$
O_{X^{\dagger} X \Psi^{\dagger} \Psi}=-4 \pi \alpha_{P Q} \frac{e_{X} e_{\Psi}}{M_{U(1)_{P Q}}^{2}} \int d^{4} \theta X^{\dagger} X \Psi^{\dagger} \Psi
$$

where in the above, $M_{U(1)_{P Q}}$ is the mass of the heavy gauge boson which is typically on the order of the GUT scale, and the $e$ 's denote the $U(1)_{P Q}$ charges of the chiral superfields $X$ and $\Psi$. When $X$ develops a supersymmetry breaking vev, this term can also contribute to the soft mass terms of the $\Psi$ fields. Depending on its precise value, this contribution can lead to an interesting predictive deformation of the gauge mediation scenario which we also study.

Although seemingly unrelated, the physics of the $X$ field and the anomalous $U(1)_{P Q}$ can also solve the strong $\mathrm{CP}$ problem. This comes about because in gauge mediated models, the phase for the scalar component of $X$ couples to the QCD instanton density through the axion-like coupling:

$$
L_{a x} \supset \frac{\arg x}{32 \pi^{2}} \varepsilon^{\mu \nu \rho \sigma} \operatorname{Tr} F_{\mu \nu} F_{\rho \sigma} .
$$

If the $U(1)_{P Q}$ symmetry had been non-anomalous, the vev of $X$ would have broken this symmetry and the corresponding Goldstone mode $\arg x$ would correspond to the longitudinal component of the massive $U(1)_{P Q}$ gauge boson, thus eliminating $\arg x$ as a candidate axion field. Precisely because this $U(1)$ symmetry is already Higgsed at high scales, a linear combination of $\arg x$ with another bulk mode axion can play the role of the QCD axion, solving the strong CP problem. A variant of this same idea for four-dimensional effective field theories has also appeared in [26], where an additional $X$ field also participates. In that model, one linear combination of the two phases corresponds to the Goldstone mode of the broken $U(1)_{P Q}$ symmetry, while another linear combination plays the role of the QCD axion.

As should now be clear, the natural appearance of an anomalous $U(1)_{P Q}$ symmetry in this class of models has many benefits. Such symmetries also fit quite 
naturally with the programme of unifying the gauge interactions of the MSSM. This fact is not new, and has been appreciated for some time in the context of fourdimensional $E_{6}$ GUT models where the chiral matter of the MSSM and Higgs fields all descend from the 27 . The $U(1)_{P Q}$ embeds as the abelian factor of the subgroup $S O(10) \times U(1) \subset E_{6}$ because the 27 decomposes as:

$$
\begin{aligned}
& E_{6} \supset S O(10) \times U(1) \\
& 27 \rightarrow 1_{+4}+10_{-2}+16_{+1}
\end{aligned}
$$

which contains both the Higgs fields $\left(10_{-2}\right)$ and the chiral matter $\left(16_{+1}\right)$ of the MSSM. Note, however, that four-dimensional models will also contain many additional states beyond those required to accommodate the MSSM. These additional states significantly reduce the appeal of starting with such large four-dimensional gauge groups.

The situation is more flexible in local F-theory models precisely because matter fields can organize into representations of a group which contains the fourdimensional gauge group as a proper subset. Geometrically, this is a consequence of the fact that the rank of the singularity type can jump by more than one rank along matter curves. When this occurs, we find that the matter content of the local F-theory GUT models typically come charged under a $U(1)$ Peccei-Quinn symmetry, rendering the matter content of our proposal technically natural. While most four-dimensional GUTs focus on the role of the 27 of $E_{6}$, we note that the messenger fields can come from the $10_{+2} \subset \overline{27}$ and the $X$ field can come from the $1_{-4} \subset \overline{27}$. One bonus feature of this scenario is that matter parity - a symmetry which is often invoked to prevent rapid nucleon decay in the MSSM - can also be identified as a $\mathbb{Z}_{2}$ subgroup of $U(1)_{P Q}$.

In any complete model of supersymmetry breaking, it is also important to specify the mechanism by which supersymmetry is broken. In keeping with the philosophy of decoupling, we present one stringy/higher dimensional gauge theoretic realization of supersymmetry breaking which only utilizes degrees of freedom associated with the $U(1)_{P Q}$ seven-brane. This $U(1)$ symmetry is anomalous and instanton effects will generate a term linear in the $X$ field which violates this symmetry. Nevertheless, this gauge symmetry leaves behind an important remnant in the form of a D-term potential with a non-trivial Fayet-Iliopoulos term determined by the background field 
strength on the Peccei-Quinn seven-brane. We find that this hybrid Fayet-Polonyi model typically breaks supersymmetry in the range required for our local F-theory models. Further details on such instanton generated effects in local F-theory models have recently been studied in [27] (see also [28]).

As noted above, the numerology of these local F-theory GUTs is remarkably constrained and we have used this fact to extract definite predictions for the low energy spectrum. We find that our model satisfies all of the required crude phenomenological constraints when:

$$
\begin{aligned}
F & \sim 10^{17} \mathrm{GeV}^{2} \\
M_{\text {mess }} & \sim x \sim 10^{12} \mathrm{GeV} \\
M_{X} & \sim 10^{15.5} \mathrm{GeV}
\end{aligned}
$$

where $M_{\text {mess }}$ is the mass of the messenger fields. Moreover we will show that obtaining these specific values does not involve any unnatural fine-tunings. The resulting values for the universal gaugino contribution $F / x, \mu$ term and axion decay constant are:

$$
\begin{aligned}
\Lambda & =\frac{F}{x} \sim 10^{5}-10^{6} \mathrm{GeV} \\
\mu & =\gamma \frac{\bar{F}}{M_{X}} \sim 10^{2}-10^{3} \mathrm{GeV} \\
f_{a} & =\sqrt{2}|x|=\sqrt{2} \frac{M_{X}}{\gamma} \frac{\mu}{\Lambda} \sim 10^{12} \mathrm{GeV},
\end{aligned}
$$

where $\gamma \sim 10$. We also find that $B \mu \sim 0$ and the A-terms vanish near the messenger scale. The small hierarchy in scales determined by the ratio $\mu / \Lambda \sim 10^{-3}$ is in fact correlated with the small hierarchy between the GUT scale and Planck scale $M_{G U T} / M_{p l} \sim 10^{-3}$. As we show in the explicit realization of the Fayet-Polonyi model, this same ratio also determines the value of the intermediate scale $10^{12} \mathrm{GeV}$.

Armed with the specific UV boundary conditions of our model, it is in fact possible to extract detailed properties of the low energy theory. The reason this is possible is primarily due to the predictive features of gauge mediation scenarios as well as the low energy requirement that electroweak symmetry breaking takes place. Even the PQ deformation away from gauge mediation is sufficiently simple to re- 
tain much of the predictive power of this scenario. Iterating all parameters under renormalization group flow between the weak scale and messenger scale allows us to constrain both the UV and IR behavior of the theory. To perform this analysis, we have used the program SOFTSUSY [29].

As in most gauge mediated scenarios, the gravitino is the LSP, and in our models it has a mass of order $10^{1}-10^{2} \mathrm{MeV}$. We find that consistent electroweak symmetry breaking requires that $\tan \beta \sim 30 \pm 7$ for $\Lambda \sim 10^{5.5 \mp 0.5} \mathrm{GeV}$. In the case of a single vector-like pair of messenger fields, the NLSP is typically a bino-like neutralino, while for a larger number of vector-like pairs ( $>3$ vector-like pairs of $5 \oplus \overline{5}$ ), the stau will instead become the NLSP. We also find that the NLSP can correspond to the stau in a single messenger model when a large PQ deformation is present.

The organization of this paper is as follows. In section 2 we briefly review some features of the local F-theory GUT models found in $[1,2]$. Next, in section 3 we review the $\mu / B \mu$ problem and in particular its relation to gauge mediated supersymmetry breaking. In section 4 we show that a broad class of local F-theory models contain Giudice-Masiero operators which require the scale of supersymmetry breaking to be lower than in gravity/moduli mediation scenarios. Motivated by this result, in section 5, we present a minimal realization of gauge mediation in this context. In section 6 we show that this class of models typically contain a candidate QCD axion with decay constant in the phenomenologically viable range. Section 7 presents a sketch of a less minimal GUT model based on embedding $U(1)_{P Q}$ in $E_{6}$, and in section 8 we study the dynamics of the anomalous $U(1)_{P Q}$ theory and show that it can break supersymmetry in the range required from bottom up considerations. We next determine in section 9 the region of MSSM parameter space for this class of models, and section 10 presents our conclusions and potential directions for future investigation.

\section{Review of Minimal F-theory GUTs}

We now briefly review the basic features of local F-theory GUT models studied in [2]. F-theory can be viewed as a strong coupling limit of type IIB string theory where the axio-dilaton is interpreted as the complex structure modulus of an elliptic curve. F-theory compactified on an elliptically fibered Calabi-Yau fourfold leads to a four-dimensional $\mathcal{N}=1$ theory below the scale of compactification. This elliptic 
fibration can degenerate to a singularity of $A D E$ type over complex codimension one subspaces of the threefold base $B_{3}$. These loci are interpreted as the location of seven-branes with corresponding gauge group of $A D E$ type. The singularity type can enhance further over complex codimension two subspaces. This is interpreted as the intersection of distinct seven-branes, which we shall refer to as matter curves. In this way, it is possible to achieve matter content such as the spinor representation of $S O(10)$, something which cannot be realized in perturbative type IIB constructions. Finally, the singularity type can enhance even further at points of $B_{3}$, where three matter curves meet. These terms lead to additional contributions to the superpotential through wave function triple overlaps. Although forbidden in perturbative type II constructions, GUT model interaction terms such as $5_{H} \times 10_{M} \times 10_{M}$ naturally occur at E-type enhancements of the compactification. See [1] for a detailed discussion of the relevant interaction terms.

As found in [2], the existence of a limit where the gauge dynamics of the GUT model can decouple from gravity turns out to impose surprisingly powerful restrictions on the ultraviolet behavior of the gauge theory. To decouple the GUT, the seven-brane must wrap a del Pezzo surface. There is essentially one such surface, called del Pezzo eight which is defined by $\mathbb{P}^{2}$ blown up at eight points. All other del Pezzo surfaces can be obtained from this one by blowing down appropriate twocycles. As shown in [1], the zero mode content for del Pezzo models never contains an adjoint valued chiral superfield, and the GUT group instead breaks to the Standard Model group through an internal flux in the hypercharge direction of the Standard Model gauge group. In these GUT models, the Higgs fields localize on curves where the net hyperflux is non-trivial, whereas the chiral matter localizes on curves with vanishing net hyperflux. In this way, general arguments based on index theory show that the chiral matter will organize into complete GUT multiplets while for an appropriate choice of flux, doublet triplet splitting will automatically occur. Index theory also requires the Higgs fields to localize on distinct curves, and this turns out to automatically forbid quartic superpotential terms responsible for proton decay. This framework is rigid enough that reliable estimates of neutrino masses can be achieved. Remarkably, the resulting values for the light neutrinos are in accord with current experimental bounds. To extract further low energy predictions, it is necessary to specify the mechanism by which supersymmetry is broken as well as the way in which this breaking communicates to the MSSM. The aim of this paper is 
to study this question in the context of gauge mediated supersymmetry breaking.

\subsection{Supersymmetry Breaking and Local Models}

Before proceeding to more specific aspects of supersymmetry breaking, we first briefly discuss the primary assumptions under which we shall operate throughout this paper. As indicated in the Introduction, the primary objective of this paper is to develop a self-consistent scenario of supersymmetry breaking in the context of a local model. Indeed, one of the advantages of working within local models is that many aspects of Planck scale physics can be deferred to a later stage of analysis. Within this framework, our goal will be to determine how various aspects of supersymmetry breaking are correlated.

On the other hand, it is well known that in some cases, issues of moduli stabilization can directly feed into aspects of supersymmetry breaking. In order for such effects not to overwhelm the contributions from purely local considerations, it is therefore necessary to assume that some supersymmetric mechanism stabilizes most moduli. It is beyond the scope of the present paper to address all aspects of moduli stabilization, and we shall therefore simply assume that an appropriate mechanism is available.

In this regard, it is worth pointing out that moduli stabilization is also intimately connected with the value of the cosmological constant. In keeping with the spirit of the present class of models where gravity can in principle decouple, we shall view our local considerations as imposing constraints on the form of candidate global models, and in particular moduli stabilization scenarios.

\section{Review of Gauge Mediation and the $\mu / B \mu$ Prob- lem}

In this section we briefly review gauge mediation and the $\mu / B \mu$ problem. This material is primarily review and can safely be skipped by the reader who is familiar

with the relevant issues. Even so, our emphasis will be slightly different than what is sometimes stressed in the literature. To frame the discussion, we first recall the $\mu / B \mu$ problem of the MSSM, and then proceed to describe how the Giudice- 
Masiero mechanism addresses this issue in gravity/moduli mediated supersymmetry breaking. Motivated by the potential presence of large FCNCs, we next review the salient features of gauge mediated supersymmetry breaking models, and explain why the $\mu / B \mu$ problem is potentially more severe in that case.

The soft supersymmetry breaking terms of the MSSM Lagrangian determine the low energy particle content. In order to solve the hierarchy problem without significant fine-tuning, the soft masses must be within an order of magnitude of the weak scale. An extensive phenomenological review of this sector of the MSSM may be found in [30]. There are typically at least three sectors in any viable model of supersymmetry breaking. These consist of the visible sector, defined by the fields and interaction terms of the MSSM, a hidden sector where supersymmetry breaking occurs, and a messenger sector which communicates the breaking of supersymmetry in the hidden sector to the visible sector. In direct mediation models, the messenger and "hidden" sectors are combined.

Supersymmetry breaking can originate from a violation of either the F-term equations of motion, the D-term equations of motion, or some combination of the two. In this paper we shall always assume that the effects of supersymmetry breaking can be parameterized by the expectation value of an MSSM gauge singlet chiral superfield $X$ with vev:

$$
\langle X\rangle=x+\theta^{2} F,
$$

so that the scale of supersymmetry breaking associated with the chiral superfield $X$ is $\sqrt{F}$. After integrating out the messenger sector, $X$ will couple to the fields of the MSSM. When $X$ attains the vev of equation (3.1), it will subsequently induce various soft supersymmetry breaking terms in the MSSM Lagrangian.

Although technically speaking the $\mu$ term of the MSSM is defined as a contribution to the superpotential of the MSSM, as we now review, the $\mu / B \mu$ problem suggests that this term and the soft supersymmetry breaking terms of the MSSM possess a common origin. Because the $H_{u}$ and $H_{d}$ fields form a vector-like pair with respect to the quantum numbers of the Standard Model gauge group $G_{s t d}$, there is a priori no reason to exclude terms in the superpotential of the MSSM of the form:

$$
W \supset \mu H_{u} H_{d} .
$$

There are various refinements of the $\mu$ problem, but at the crudest level, it is the 
puzzling fact that the mass of a vector-like pair is generically closer to the GUT scale rather than the weak scale. Similarly, there is no reason to exclude large terms in the effective potential involving the scalar components $h_{u}$ and $h_{d}$ of the respective chiral superfields $H_{u}$ and $H_{d}$ :

$$
V_{e f f} \supset B \mu h_{u} h_{d}
$$

Combined, these two issues define the weakest version of the $\mu / B \mu$ problem.

At the level of effective field theory, the bare $\mu$ term can be forbidden by assuming that the Higgs superfields are both charged under a $U(1)$ Peccei-Quinn symmetry. In order to allow all of the necessary interaction terms of the MSSM, all fields which couple to the Higgs fields must also be appropriately charged under $U(1)_{P Q}$. As explained in [2], such symmetries are quite common in GUT models based on Ftheory.

In F-theory, vector-like pairs interact by coupling with gauge singlet fields localized on matter curves normal to the surface wrapped by the GUT model seven-brane. For example, letting $X_{\perp}$ denote the singlet which interacts with the Higgs fields, the corresponding contribution to the superpotential is:

$$
W_{X_{\perp} H_{u} H_{d}}=\kappa X_{\perp} H_{u} H_{d}
$$

Depending on the sign of fluxes through the curve supporting the $X_{\perp}$ field, the singlet wave function will either be attracted or repelled away from the GUT model seven-brane. When it is repelled, the value of the Yukawa coupling $\kappa$ can naturally be much smaller than an order one number, so that even if the vev of $X_{\perp}$ is near the GUT scale, the resulting $\mu$ term could still be quite small. Note in particular that $B=0$ at high scales, and is only generated at lower energies by radiative corrections to the effective potential for the Higgs scalars.

Even so, this solution is somewhat unsatisfactory because it does not explain why $\mu$ is so close to the scale of supersymmetry breaking. This is a stronger version of the $\mu / B \mu$ problem. On the other hand, it could well be that these two scales are simply uncorrelated, in which case the above mechanism provides a simple mechanism by which to solve the weak version of the $\mu$ problem. Solving the strong version of the $\mu / B \mu$ problem is one of the primary aims of this paper.

Beyond aesthetic concerns, there is a potentially more serious problem when the coupling of equation (3.4) contributes to the superpotential. Indeed, when $X_{\perp}=X$, 
note that the vev of equation (3.1) will generate contributions to the $\mu$ and $B \mu$ terms:

$$
\begin{aligned}
\mu & =\kappa x \\
B \mu & =\kappa F .
\end{aligned}
$$

In other words, when $\kappa$ is an order one coefficient, the vevs of $x$ and $\sqrt{F}$ must already be quite close to the weak scale to avoid removing the Higgs fields from the low energy spectrum. This is problematic because as we will review shortly, in both gravity/moduli mediation and gauge mediation scenarios, $\sqrt{F}$ is typically greater than the weak scale.

\subsection{Interpolating from Gravity/Moduli Mediation to Gauge Mediation}

Starting from higher values of the supersymmetry breaking scale set by $\sqrt{F}$, we now review how a phenomenologically viable mediation mechanism will interpolate from gravity/moduli mediation to gauge mediation at lower values of $\sqrt{F}$. One conveniant way to parameterize the dominant mediation mechanism is in terms of the gravitino mass:

$$
m_{3 / 2}=\sqrt{\frac{4 \pi}{3}} \frac{|F|}{M_{p l}}
$$

where $M_{p l} \sim 1.2 \times 10^{19} \mathrm{GeV}$. In gravity mediation models, the gravitino mass is around $10^{2}-10^{3} \mathrm{GeV}$, while in gauge mediation models, this value is at the very most $1 \mathrm{GeV}$. In certain cases, it is possible to further increase the scale of supersymmetry breaking in anomaly mediated scenarios. For further review, see for example, [21].

Gravity mediated supersymmetry breaking refers to any class of models where the soft-breaking terms originate from Planck suppressed higher-dimension operators. As such, in string theory it is more appropriate to refer to this class of possibilities as gravity/moduli mediated supersymmetry breaking. The $X$ field couples to the chiral superfields $\Psi$ of the MSSM through terms such as:

$$
L_{\text {soft }} \supset \int d^{4} \theta\left(\gamma_{p l} \frac{X^{\dagger} H_{u} H_{d}}{M_{p l}}+\gamma_{\Psi} \frac{X^{\dagger} X \Psi^{\dagger} \Psi}{M_{p l}^{2}}\right)
$$


where the $\gamma$ 's denote order one coefficients. When $X$ develops a vev as in equation (3.1), the resulting theory will automatically contain a contribution to the $\mu$-term and the soft scalar mass terms:

$$
\begin{gathered}
\mu_{e f f} \sim \frac{\bar{F}}{M_{p l}} \\
m_{\Psi}^{2} \sim \frac{|F|^{2}}{M_{p l}^{2}} .
\end{gathered}
$$

In other words, the scale $\mu_{e f f}$ correlates with the energy scale of the soft mass terms, solving the $\mu$-problem. This means of generating the $\mu$ term is known as the Giudice-Masiero mechanism [14] and we shall sometimes refer to $X^{\dagger} H_{u} H_{d}$ as the Giudice-Masiero operator. Note that by construction, the value of $\mu$ correlates with the scale of supersymmetry breaking.

In order for the Higgs up/down fields to retain masses near the weak scale, the contribution to the $\mu$ term from the Giudice-Masiero mechanism must be at most $10^{3} \mathrm{GeV}$. Hence, $|F|$ is bounded above by 2

$$
|F| \lesssim 10^{21}-10^{22} \mathrm{GeV}^{2}
$$

It is certainly appealing that in gravity/moduli mediated models, the $\mu$ term automatically correlates with the scale of the soft breaking terms. But in gravity/moduli mediated scenarios, the coefficients $\gamma_{\Psi}$ in equation (3.8) are also typically generic order one coefficients, so that large flavor changing neutral currents (FCNCs) will be present. For this reason, it is quite common to specify additional flavor symmetries in order for such models to remain viable.

Nevertheless, the Planck suppressed contributions proportional to $\gamma_{\Psi}$ in equation (3.8) will always be present, and in the absence of an appropriate theory of flavor, will always generate FCNCs when $X$ develops a supersymmetry breaking vev. Perhaps the simplest way to avoid any problem with FCNCs is to lower the scale of supersymmetry breaking so that $F / M_{p l}$ is at most $1 \mathrm{GeV}$. As reviewed in [21], for example, this bound is low enough to remain in accord with observation.

If the contribution of the Planck suppressed operators to the soft mass terms falls

\footnotetext{
${ }^{2}$ We note that this value can be increased even further in certain scenarios such as anomaly mediation.
} 
below the weak scale, other mediation mechanisms must account for the soft breaking terms of the MSSM. Gauge mediation [31-40] is a mechanism where the gauge fields of the Standard Model communicate supersymmetry breaking to the MSSM. See [41] for a review of gauge mediation. In gauge mediation, the $X$ field couples in the superpotential to "messenger fields" which either transform in a real representation of the Standard Model gauge group $G_{s t d}=S U(3)_{C} \times S U(2)_{L} \times U(1)_{Y}$, or in vectorlike pairs of complex representations. In this paper we shall always assume that the messenger fields transform in vector-like pairs of complex representations, and we shall denote these pairs as $Y$ and $Y^{\prime}$.

As a brief aside, we note that this simplifying assumption is well-motivated in F-theory models. For example in the minimal $S U(5)$ GUT models studied in [2], the only available representations are the 24 for bulk modes, and the 5, 10 or complex conjugates for modes which localize on matter curves. Furthermore, when the GUT model seven-brane wraps a del Pezzo surface, the vanishing theorem of [1] establishes that no zero modes transform in the 24 of $S U(5)$. Hence, all chiral superfields of the four-dimensional effective theory charged under the GUT group must descend from complex representations of $S U(5)$.

In this paper we shall further restrict attention to single messenger models where the gauge singlet $X$ interacts with the messenger fields through the superpotential term:

$$
W_{X Y Y}=\lambda X Y Y^{\prime}
$$

where $\lambda$ is generically an order one coefficient 3 Even in this simple case, there is additional structure present in most F-theory models because the wave functions of different components of a complete GUT representation will in general be different [2].

More generally, it is in principle possible to consider models with a larger number of messenger fields. On the other hand, in order to maintain the existence of a decoupling limit, we must also require that the running of the couplings in the zero mode sector should preserve asymptotic freedom. In an $S U(5)$ model with three generations in the $\overline{5}_{M} \oplus 10_{M}$, a single vector-like pair of Higgs fields and $N_{\text {mess }}$

\footnotetext{
${ }^{3}$ While it is certainly possible to also consider models where the messenger sector is strongly coupled, in the context of F-theory models, our expectation is that a perturbative treatment will suffice for most purposes. The study of strongly coupled messenger sectors is indeed a topic of current interest, see for example $[42,43]$. In the context of local F-theory models with perturbative gauge dynamics, we shall make the reasonable assumption that the messenger sector is sufficiently weakly coupled that a perturbative treatment is available.
} 
vector-like pairs of messengers in the $5 \oplus \overline{5}$ of $S U(5)$, the beta function is:

$$
\begin{aligned}
b_{G U T} & =3 C_{2}(24)-3\left(C_{2}\left(10_{M}\right)+C_{2}\left(\overline{5}_{M}\right)\right)-2 C_{2}\left(5_{H}\right)-2 N_{\text {mess }}\left(C_{2}\left(5_{Y}\right)\right) \\
& =8-N_{\text {mess }}
\end{aligned}
$$

where in the above, $C_{2}$ denotes the quadratic Casimir of various representations. In other words, asymptotic freedom limits the number of messengers to $N_{\text {mess }}<8$. This is somewhat stronger than the usual condition typically considered in the GUT literature where the coupling constant must simply remain perturbative up to the GUT scale.

Once $X$ develops a vev as in equation (3.1), the coupling between the gauge fields and the messenger fields will induce soft breaking terms in the MSSM. Integrating out the $Y$ fields, the soft breaking terms will contain the contributions:

$$
\begin{aligned}
L & \supset \int d^{4} \theta\left(\sum_{i=1}^{3}-\alpha_{i}^{2} C_{2}\left(R_{\Psi}^{i}\right)\left(\log |X|^{2}\right)^{2} \Psi^{\dagger} \Psi\right) \\
& +\int d^{2} \theta \operatorname{Re}\left(\sum_{i=1}^{3} \frac{1}{8 \pi i}\left(\tau_{Y M}^{(i)}+\frac{1}{2 \pi i} \log X\right) \operatorname{Tr}_{G_{(i)}} W_{(i)}^{\alpha} W_{(i) \alpha}\right)
\end{aligned}
$$

where $\Psi$ is shorthand for any chiral superfield of the MSSM, and $\tau_{Y M}^{(i)}$ denotes the holomorphic Yang-Mills coupling of the $i^{\text {th }}$ gauge group factor $G_{(i)}$ so that:

$$
\tau_{Y M}=\frac{4 \pi i}{g_{Y M}^{2}}+\frac{\theta_{Y M}}{2 \pi}=\frac{i}{\alpha_{Y M}}+\frac{\theta_{Y M}}{2 \pi} .
$$

In gauge mediation, the soft masses of the gauginos follow from equation (3.16) so that:

$$
m_{i}=\frac{\alpha_{i}}{4 \pi} \frac{F}{x} \equiv \frac{\alpha_{i}}{4 \pi} \Lambda
$$

where in the above, we have introduced the ratio $\Lambda=F / x$. Assuming that the mass of the bino is on the order of $10^{2} \mathrm{GeV}$ and originates predominantly from gauge mediation requires:

$$
\Lambda=\frac{F}{x} \sim 10^{5} \mathrm{GeV}
$$

One of the most important features of gauge mediation is that in the absence of 
other sources of flavor violation, the soft masses depend only on the gauge quantum numbers of a given field. In particular, this means that the potentially dangerous FCNCs of gravity mediated models are quite suppressed.

Turning the discussion around, the presence of messenger fields in the low energy spectrum could in principle be incompatible with a higher scale of supersymmetry breaking such as that associated with gravity/moduli mediation. For example, if the singlet field $X$ interacts with messenger fields transforming in GUT multiplets, increasing the ratio $\Lambda=F / x$ will simply increase the contribution to the soft masses due to gauge mediation.

Unfortunately, generating an appropriate value of the $\mu$ term in gauge mediation is somewhat problematic. Indeed, if the only contribution to the effective $\mu$ term is given by equation (3.9), the resulting value of $\mu$ would be far too low. The simplest attempts to solve the $\mu / B \mu$ problem also fail. For example, returning to the discussion near equation (3.5), directly coupling the $X$ field to the Higgs fields through a term such as:

$$
W_{X H_{u} H_{d}}=\kappa X H_{u} H_{d}
$$

can indeed induce an appropriate value for the $\mu$ term. Note, however, that $B$ is insensitive to the value of $\kappa$ because:

$$
B=\frac{B \mu}{\mu}=\frac{\kappa F}{\kappa x}=10^{5} \mathrm{GeV} .
$$

Thus, unless $\kappa$ is quite small, generic values of $x$ and $F$ will typically generate mass terms for the Higgs fields far above the weak scale. While it is indeed possible in F-theory constructions to exponentially suppress $\kappa$ so that both these terms are sufficiently small, the alternative and conceptually simpler point of view which we shall adopt in this paper is that the coupling of line (3.20) should be absent from the low energy superpotential.

\section{Variants of the Giudice-Masiero Mechanism and Higher Dimension Operators}

Although the Giudice-Masiero mechanism solves the $\mu$ problem in gravity/moduli mediated scenarios, in gauge mediated models, the same contribution to the $\mu$ term 
would be far below the weak scale. But while this contribution to the low energy theory is somewhat innocuous, we have also seen that the superpotential term $X H_{u} H_{d}$ should not be present. In models with a $U(1)_{P Q}$ symmetry, the Giudice-Masiero mechanism and the presence of the term $X H_{u} H_{d}$ are in fact mutually exclusive. Indeed, when $H_{u}, H_{d}$ and $X$ are all charged under $U(1)_{P Q}$, the F-term $X H_{u} H_{d}$ is forbidden whenever the D-term $X^{\dagger} H_{u} H_{d}$ is allowed, and the converse statement holds as well. Thus, the existence of the Giudice-Masiero operator effectively frustrates the $B \mu$ term.

The Giudice-Masiero operator:

$$
O_{X^{\dagger} H_{u} H_{d}}=\gamma_{p l} \int d^{4} \theta \frac{X^{\dagger} H_{u} H_{d}}{M_{p l}}
$$

can also generate a value of $\mu$ near the weak scale in gauge mediated scenarios when the coefficient $\gamma_{p l}$ is sufficiently large. Said differently, this is equivalent to replacing $\gamma_{p l}$ by an order one coefficient and the suppression scale $M_{p l}$ by some lower energy scale $M_{X}$, such as the GUT scale so that:

$$
O_{X^{\dagger} H_{u} H_{d}}=\gamma \int d^{4} \theta \frac{X^{\dagger} H_{u} H_{d}}{M_{X}}
$$

This observation is not new and has, for example, formed the basis of the "sweet spot" model of supersymmetry breaking [21].

In a generic effective field theory, the typical situation is not as simple. If we perform the natural identification $M_{X}=x$, this theory will also generate a large $B \mu$ term via the operator:

$$
\int d^{4} \theta \frac{X^{\dagger} X X^{\dagger} H_{u} H_{d}}{M_{X}^{3}} .
$$

Indeed, saturating the $d^{4} \theta$ integrals through the $X$ superfields yields:

$$
B \mu=-\frac{|F|^{2} \bar{x}}{M_{X}^{3}} .
$$

When $M_{X}=|x|$, this yields a problematically large value of the $B \mu$ term:

$$
|B \mu|=\left|\frac{F}{x}\right|^{2}
$$


Thus, in order to solve the $\mu / B \mu$ problem, we must also explain why the suppression scale in the above operators can be greater in magnitude than $x$.

Moreover, while intriguing, replacing $M_{p l}$ by $M_{X}$ in a four-dimensional effective field theory is quite ad hoc. Nevertheless, in this paper we will show that this replacement is quite natural in local F-theory models and in certain cases unavoidable. For example, when $F \sim 10^{17} \mathrm{GeV}^{2}$ and $M_{X} \sim 10^{15.5} \mathrm{GeV}$, this induces an effective $\mu$ term:

$$
\mu_{\text {eff }}=\gamma \frac{\bar{F}}{M_{X}} \sim \gamma \cdot 10^{1.5} \mathrm{GeV}
$$

which without any fine-tuning is already quite close to the weak scale. Further, as we show later in this paper, fluxes can naturally allow the scale of $|x|$ to be much smaller than $M_{X}$. Indeed, the mild hierarchy of scales $|x| / M_{X} \sim 10^{-3}$ provides a solution to the $B \mu$ problem in the class of models we consider.

Summarizing the bottom up considerations described above, we are interested in four-dimensional effective field theories where the F-term coupling $X H_{u} H_{d}$ is forbidden, but the D-term coupling $X^{\dagger} H_{u} H_{d}$ is allowed. In F-theory constructions, these two features are not only compatible, but are in fact quite tightly correlated! Geometrically, the matter curves supporting the $X, H_{u}$ and $H_{d}$ fields must form a triple intersection in order for the D-term coupling to be gauge-invariant. Remarkably, integrating out the Kaluza-Klein modes associated with $X$ generates a Giudice-Masiero operator suppressed by an energy scale close to $M_{G U T}$.

This has important consequences for F-theory models where the $X$ field is charged under a Peccei-Quinn symmetry and interacts with the Higgs fields through a GiudiceMasiero term. Because the suppression scale of this operator will at most be a few orders of magnitude below the Planck scale, it follows that the resulting $\mu$ term would be far too large in gravity/moduli mediated scenarios. In other words, we deduce that in a large class of F-theory compactifications, the scale of supersymmetry breaking must be sufficiently low to remain in accord with electroweak symmetry breaking!

The presence of the $U(1)_{P Q}$ symmetry has another consequence in the low energy theory. Whereas in a four-dimensional effective field theory $U(1)_{P Q}$ can be treated as a global symmetry, in a quantum theory of gravity, this symmetry must be gauged. When this $U(1)$ is non-anomalous, it will also contribute to the soft scalar mass terms via the usual gauge mediation mechanism. However, in F-theory constructions, 
this $U(1)$ is typically anomalous, and the corresponding gauge boson will develop a large mass via the Green-Schwarz mechanism. Precisely because all of the fields of the MSSM must be charged under $U(1)_{P Q}$, heavy $U(1)_{P Q}$ gauge boson exchange between MSSM fields and $X$ will generate additional contributions to the soft scalar mass terms of the MSSM fields.

The rest of this section is organized as follows. First, we show that in local F-theory models where the $U(1)_{P Q}$ symmetry allows the $X$ field to couple to the Higgs fields through a Giudice-Masiero operator, integrating out the Kaluza-Klein modes of the higher dimensional theory automatically generates this operator. This establishes that in a large class of F-theory models, gravity/moduli mediation would yield a value of the $\mu$ term which is phenomenologically unviable. Next, we show that when the $U(1)_{P Q}$ symmetry is anomalous, heavy $U(1)_{P Q}$ gauge boson exchange can in some cases generate important corrections to the soft scalar masses.

\subsection{Giudice-Masiero Operators}

In this subsection we show that in a broad class of F-theory models, integrating out the Kaluza-Klein modes associated with $X$ generates a Giudice-Masiero term which is suppressed by the Kaluza-Klein scale. We emphasize that this is essentially a tree level computation, and that once additional details of the compactification have been specified, the coefficient of this higher dimension operator is completely calculable. This is in contrast to standard arguments from effective field theory, which must typically appeal to estimates based on genericity considerations.

To generate a Giudice-Masiero operator, we shall assume that the matter curves $\Sigma_{X}, \Sigma_{H_{u}}$ and $\Sigma_{H_{d}}$ which support the fields $X, H_{u}$ and $H_{d}$ form a triple intersection such that $X H_{u} H_{d}$ is not a gauge invariant operator. In this case, the term:

$$
O_{X^{\dagger} H_{u} H_{d}}=\gamma \int d^{4} \theta \frac{X^{\dagger} H_{u} H_{d}}{M_{X}}
$$

does correspond to a gauge invariant operator. This is because, as explained in [1], the six-dimensional fields from which $X, H_{u}$ and $H_{d}$ descend organize into vectorlike pairs of four-dimensional $\mathcal{N}=1$ chiral superfields $\mathbb{X} \oplus \mathbb{X}^{c}, \mathbb{H}_{u} \oplus \mathbb{H}_{u}^{c}$ and $\mathbb{H}_{d} \oplus \mathbb{H}_{d}^{c}$ labelled by points on the matter curves. Thus, only one of the two interaction terms $\mathbb{X H}_{u} \mathbb{H}_{d}$ or $\mathbb{X}^{c} \mathbb{H}_{u} \mathbb{H}_{d}$ can descend to a superpotential term for the zero modes. 
Assuming the $\mathbb{X}^{c} \mathbb{H}_{u} \mathbb{H}_{d}$ interaction is present, the fact that $\mathbb{X}^{\dagger}$ and $\mathbb{X}^{c}$ have identical gauge quantum numbers implies that $O_{X^{\dagger} H_{u} H_{d}}$ is gauge invariant. To establish that $O_{X^{\dagger} H_{u} H_{d}}$ is generated, we first present the Lagrangian density for this system in four-dimensional $\mathcal{N}=1$ superspace:

$$
\begin{aligned}
L & =\int_{\Sigma_{X}} d^{4} \theta\left[\mathbb{X}^{\dagger} e^{V^{\prime}} \mathbb{X} e^{V^{\prime \prime}}+\left(\mathbb{X}^{c}\right)^{\dagger} e^{-V^{\prime}} \mathbb{X}^{c} e^{-V^{\prime \prime}}\right] \\
& +\int_{\Sigma_{u}} d^{4} \theta\left[\left(\mathbb{H}_{u}\right)^{\dagger} e^{V^{\prime}} \mathbb{H}_{u} e^{+V}+\left(\mathbb{H}_{u}^{c}\right)^{\dagger} e^{-V^{\prime}} \mathbb{H}_{u}^{c} e^{-V}\right] \\
& +\int_{\Sigma_{d}} d^{4} \theta\left[\left(\mathbb{H}_{d}\right)^{\dagger} e^{+V^{\prime \prime}} \mathbb{H}_{d} e^{-V}+\left(\mathbb{H}_{d}^{c}\right)^{\dagger} e^{-V^{\prime \prime}} \mathbb{H}_{d}^{c} e^{+V}\right] \\
& +\int_{\Sigma_{X}} d^{2} \theta \mathbb{X}^{c}\left(\bar{\partial}+\mathbb{A}^{\prime}+\mathbb{A}^{\prime \prime}\right) \mathbb{X} \\
& +\int_{\Sigma_{u}} d^{2} \theta \mathbb{H}_{u}^{c}\left(\bar{\partial}+\mathbb{A}+\mathbb{A}^{\prime}\right) \mathbb{H}_{u} \\
& +\int_{\Sigma_{d}} d^{2} \theta \mathbb{H}_{d}^{c}\left(\bar{\partial}+\mathbb{A}-\mathbb{A}^{\prime \prime}\right) \mathbb{H}_{d} \\
& +\int_{\Sigma} d^{2} \theta\left[\delta_{p} \mathbb{X}^{c} \mathbb{H}_{u} \mathbb{H}_{d}+\delta_{p} \mathbb{X} \mathbb{H}_{u}^{c} \mathbb{H}_{d}^{c}\right]+h . c . .
\end{aligned}
$$

In the above, we have organized the gauge field contribution from the various sevenbranes into four-dimensional $\mathcal{N}=1$ supermultiplets so that the $V$ 's denote the contribution from vector multiplets which transform as bulk mode scalars on the associated Kähler surface and the $\mathbb{A}$ 's denote the contribution from chiral superfields which transform as bulk mode one-forms on the appropriate Kähler surface. Finally, $\delta_{p}$ denotes a delta function with support at a point of triple intersection. The F-term equations of motion for the six-dimensional fields are:

$$
\begin{aligned}
& \frac{\partial W}{\partial \mathbb{X}}=-\bar{\partial}_{\mathbb{A}^{\prime}+\mathbb{A}^{\prime \prime}} \mathbb{X}^{c}+\delta_{p} \mathbb{H}_{u}^{c} \mathbb{H}_{d}^{c}=0 \\
& \frac{\partial W}{\partial \mathbb{X} \mathbb{X}^{c}}=\bar{\partial}_{\mathbb{A}^{\prime}+\mathbb{A}^{\prime \prime}} \mathbb{X}+\delta_{p} \mathbb{H}_{u} \mathbb{H}_{d}=0
\end{aligned}
$$


with similar expressions for the $\mathbb{H}$ equations of motion. Expanding about a fixed supersymmetric background gauge field configuration for the fields $\mathbb{A}^{\prime}$ and $\mathbb{A}^{\prime \prime}$ which we denote by $A^{\prime}$ and $A^{\prime \prime}$, solving for $\mathbb{X}^{c}$ and $\mathbb{X}$ yields:

$$
\begin{aligned}
\mathbb{X}^{c} & =\frac{1}{\bar{\partial}_{A^{\prime}+A^{\prime \prime}}}\left(\delta_{p} \mathbb{H}_{u}^{c} \mathbb{H}_{d}^{c}\right) \\
\mathbb{X} & =X-\frac{1}{\bar{\partial}_{A^{\prime}+A^{\prime \prime}}}\left(\delta_{p} \mathbb{H}_{u} \mathbb{H}_{d}\right)
\end{aligned}
$$

where in the above, we have included the zero mode $X$ which by definition, is annihilated by $\bar{\partial}_{A^{\prime}+A^{\prime \prime}}$.

Substituting these expressions into equation (4.8) is equivalent to integrating out the Kaluza-Klein modes of the $\mathbb{X}$ and $\mathbb{X}^{c}$ fields. The resulting effective action for the zero modes therefore contains the term:

$$
\begin{aligned}
L_{e f f} & \supset \int_{\Sigma_{X}} d^{4} \theta\left[\left(X-G_{A^{\prime}+A^{\prime \prime}}(z, p) H_{u} H_{d}\right)^{\dagger} e^{V^{\prime}}\left(X-G_{A^{\prime}+A^{\prime \prime}}(z, p) H_{u} H_{d}\right) e^{V^{\prime \prime}}\right] \\
& +\int_{\Sigma_{u}} d^{4} \theta\left[H_{u}^{\dagger} e^{V^{\prime}} H_{u} e^{+V}\right]+\int_{\Sigma_{d}} d^{4} \theta\left[H_{d}^{\dagger} e^{+V^{\prime \prime}} H_{d} e^{-V}\right]
\end{aligned}
$$

where in the above, $G_{A^{\prime}+A^{\prime \prime}}$ denotes the Green's function defined by the relation:

$$
\bar{\partial}_{A^{\prime}+A^{\prime \prime}} G_{A^{\prime}+A^{\prime \prime}}(z, p)=\delta_{p}=\delta^{(2)}(z-p)
$$

where $z$ is a local coordinate on the Riemann surface $\Sigma_{X}$. Using the approximation:

$$
\int_{\Sigma_{X}} G_{A^{\prime}+A^{\prime \prime}}(z, p) \sim \frac{M_{*}^{2} \operatorname{Vol}\left(\Sigma_{X}\right)}{M_{X}}
$$

where $M_{X}$ denotes the mass scale of the Kaluza-Klein modes on the curve $\Sigma_{X}$, canonically normalizing all Kinetic terms yields the Lagrangian density of the $X /$ Higgs 
system:

$$
\begin{aligned}
L_{e f f} & \supset \int d^{4} \theta\left[H_{u}^{\dagger} e^{V^{\prime}} H_{u} e^{+V}+H_{d}^{\dagger} e^{+V^{\prime \prime}} H_{d} e^{-V}+X^{\dagger} e^{V^{\prime}} X e^{-V^{\prime \prime}}\right] \\
& +\int d^{4} \theta\left[\frac{\sqrt{M_{*}^{2} \operatorname{Vol}\left(\Sigma_{X}\right)}}{\sqrt{M_{*}^{2} \operatorname{Vol}\left(\Sigma_{u}\right)} \sqrt{M_{*}^{2} \operatorname{Vol}\left(\Sigma_{d}\right)}} \frac{X^{\dagger} e^{V^{\prime}} H_{u} H_{d} e^{V^{\prime \prime}}}{M_{X}}\right]+\text { h.c.. }
\end{aligned}
$$

When $X$ develops a non-supersymmetric vev, this induces an effective $\mu$-term:

$$
\mu_{e f f}=\frac{\sqrt{M_{*}^{2} \operatorname{Vol}\left(\Sigma_{X}\right)}}{\sqrt{M_{*}^{2} \operatorname{Vol}\left(\Sigma_{u}\right)} \sqrt{M_{*}^{2} \operatorname{Vol}\left(\Sigma_{d}\right)}} \times \frac{\bar{F}}{M_{X}} \equiv \gamma \frac{\bar{F}}{M_{X}} .
$$

A similar expression holds when additional $X$ field zero modes contribute to the low energy theory. Assuming that $\operatorname{Vol}\left(\Sigma_{u}\right)=\operatorname{Vol}\left(\Sigma_{d}\right)=M_{G U T}^{-2}$, the relations $M_{*}^{4} / M_{G U T}^{4}=\alpha_{G U T}$ and $\operatorname{Vol}\left(\Sigma_{X}\right)=M_{X}^{-2}$ imply:

$$
\gamma=\alpha_{G U T}^{1 / 4} \frac{M_{G U T}}{M_{X}}
$$

As estimated in [2], it is most natural for the Kaluza-Klein scale $M_{X}$ be close to the GUT scale. When convenient, throughout this paper we will use the representative values $M_{G U T} \sim 3 \times 10^{16} \mathrm{GeV}$ and $M_{X} \sim 10^{15.5} \mathrm{GeV}$. This implies that the parameter $\gamma \sim 10$, and that $\mu_{\text {eff }} \sim 300 \mathrm{GeV}$. Thus, this class of higher-dimensional models realizes a variant of the Giudice-Masiero mechanism, but for gauge mediated models!

To close this section, let us also address the value of the $B \mu$ term in this case. Due to the presence of the $U(1)_{P Q}$ symmetry, the leading order contribution originates from the higher dimension operator:

$$
\int d^{4} \theta \frac{X^{\dagger} X X^{\dagger} H_{u} H_{d}}{M_{X}^{3}} \rightarrow \mu \frac{\bar{x}}{M_{X}} h_{u} h_{d}
$$

where $h_{u}$ and $h_{d}$ denote the scalar components of the Higgs chiral superfields. Since $\bar{x} / M_{X}$ is by construction small, the resulting value of $B \mu$ at the messenger scale can effectively be set to zero. In addition, let us also note that additional corrections to $B \mu$ will be suppressed because the matter fields localize on curves in the higher dimensional geometry. Similar considerations apply for other contributions, such as the A-terms of the MSSM. 


\section{2 $U(1)_{P Q}$ Induced Soft Mass Terms}

In a quantum theory of gravity, the $U(1)$ Peccei-Quinn symmetry must be gauged. Thus, because the $X$ field is charged under $U(1)_{P Q}$, it will interact via gauge boson exchange with all other fields charged under $U(1)_{P Q}$. In the present case of interest, the $U(1)_{P Q}$ symmetry is anomalous and the corresponding gauge boson will develop a large mass via the Green-Schwarz mechanism. Heavy $U(1)_{P Q}$ gauge boson exchange generates the operators [44]:

$$
O_{X^{\dagger} X \Psi^{\dagger} \Psi}=-4 \pi \alpha_{P Q} \frac{e_{X} e_{\Psi}}{M_{U(1)_{P Q}}^{2}} \cdot \int d^{4} \theta X^{\dagger} X \Psi^{\dagger} \Psi
$$

where in the above, $\Psi$ denotes a generic chiral superfield charged under $U(1)_{P Q}$, and $X$ and $\Psi$ are canonically normalized chiral superfields. In addition, $e_{X}=+4$, $e_{\Psi}=+2$ for the Higgs fields, and $e_{\Psi}=-1$ for all other chiral superfields of the MSSM. An important feature of this contribution is that the overall sign of the corresponding operator is completely fixed by the charges of the various fields. In addition, this term is diagonal in a gauge quantum number basis of eigenstates. This implies that this class of operators will not introduce additional FCNCs. Finally, we note that we have implicitly assumed that the dominant contribution to this operator comes from this heavy $U(1)_{P Q}$ gauge boson. In higher dimensional theories such as this one, additional terms could potentially yield corrections to this result when the mass of this gauge boson is sufficiently large.

Once $X$ develops a supersymmetry breaking vev, the operator of equation (4.28) will induce a contribution to the soft masses squared:

$$
\delta_{P Q} m_{\Psi}^{2}\left(M_{m e s s}\right)=4 \pi \alpha_{P Q} \frac{e_{\Psi} e_{X}}{M_{U(1)_{P Q}}^{2}}|F|^{2} .
$$

Note that the relative strengths of this contribution to the matter fields versus Higgs fields is set by their relative PQ charges:

$$
\frac{\delta_{P Q} m_{\Phi}^{2}\left(M_{\text {mess }}\right)}{\delta_{P Q} m_{H}^{2}\left(M_{\text {mess }}\right)}=-\frac{1}{2}
$$

where here, $H$ refers to the Higgs superfields and $\Phi$ refers to all other chiral superfields of the MSSM. This provides a universal prediction for a specific deformation 
away from the usual gauge mediated supersymmetry breaking scenario 4 In particular, we also note that the PQ deformation will lower the mass of the sleptons and squarks, and will increase the soft mass squared of the Higgs fields at the messenger scale. Note that to leading order, the masses of the gauginos are not altered by this contribution 5

As a crude order of magnitude estimate, we use the values for $\alpha_{P Q}$ and $M_{U(1)_{P Q}}$ for external $U(1)$ factors obtained in [2]:

$$
\begin{aligned}
\alpha_{P Q} & \sim 10^{-2} \\
M_{U(1)_{P Q}} & \sim 10^{15} \mathrm{GeV} .
\end{aligned}
$$

Assuming that $F \leq 10^{17} \mathrm{GeV}^{2}$, the soft mass terms are roughly given by:

$$
\Delta_{P Q} \sim \sqrt{4 \pi \alpha_{P Q}} \frac{F}{M_{U(1)_{P Q}}} \sim 30 \mathrm{GeV}
$$

These estimates depend on various assumptions about the sizes of $\alpha_{P Q}$ and $M_{U(1)_{P Q}}$, and a slight change in their values can potentially induce important contributions to the soft mass terms of the MSSM. Indeed, we will study some of the consequences of this deformation in section 9 .

\section{$5 \quad$ Diamond Ring Model}

In this section we present a minimal F-theory realization of gauge mediated supersymmetry breaking where $X$ couples to the Higgs fields through a Giudice-Masiero operator. As we have seen in previous sections, the presence of an additional $U(1)_{P Q}$ symmetry plays an especially prominent role in the low energy effective field theory. In F-theory, such symmetries can originate from either Kaluza-Klein reduction of bulk gravity modes, or from the worldvolume gauge group of seven-branes in the

\footnotetext{
${ }^{4}$ That this is the leading contribution to this higher dimension operator follows from the fact that the $X$ and $\Psi$ fields localize on different curves.

${ }^{5}$ As emphasized in [44], there will in general be a contribution to the masses of the gauginos in such models via D-term breaking effects through the coupling of the gauge fields to the dilaton. Typically, however, such contributions can only make a substantial contribution to the gaugino masses when the value of $F$ is larger than we consider here, and we shall therefore neglect this contribution throughout this paper.
} 
compactification. In keeping with the general principle of decoupling, we shall only consider the latter possibility.

We consider a model where $X$ localizes on a matter curve defined by the intersection of two distinct seven-branes wrapping Kähler surfaces which we denote by $S^{\prime}$ and $S^{\prime \prime}$. To denote the charge of $X$ under these two seven-branes which generically have $U(1)$ gauge groups, we shall sometimes write $X^{+,-}$. In order for $X^{+,-}$to interact with both the messenger fields and the Higgs fields, the curve $\Sigma_{X}$ must form one triple intersection with the messenger curves and another triple intersection with the Higgs curves.

In the four-dimensional effective field theory, the superpotential coupling $X Y Y^{\prime}$ is allowed and the coupling $X H_{u} H_{d}$ is forbidden when the $Y, H$ and GUT model chiral matter fields have charges:

\begin{tabular}{|r|r|r|r|}
\hline & $U(1)^{\prime}$ & $U(1)^{\prime \prime}$ & $U(1)_{P Q}$ \\
\hline$X$ & +2 & -2 & +4 \\
\hline$Y$ & -2 & 0 & -2 \\
\hline$Y^{\prime}$ & 0 & +2 & -2 \\
\hline$H_{u}$ & +2 & 0 & +2 \\
\hline$H_{d}$ & 0 & -2 & +2 \\
\hline $10_{M}$ & -1 & 0 & -1 \\
\hline$\overline{5}_{M}$ & +1 & +2 & -1 \\
\hline
\end{tabular}

where in the above, we have also defined the $U(1)_{P Q}$ charge as the linear combination:

$$
U(1)_{P Q}=U(1)^{\prime}-U(1)^{\prime \prime}
$$

Geometrically, the curve $\Sigma_{X}$ must intersect $S$ at two distinct points. For this reason, we shall refer to this construction as the "diamond ring model". See figure 1 for a depiction of this intersecting brane configuration.

By construction, the low energy effective theory contains the terms:

$$
L \supset \gamma \int d^{4} \theta \frac{X^{\dagger} H_{u} H_{d}}{M_{X}}+\int d^{2} \theta \lambda X Y Y^{\prime}
$$




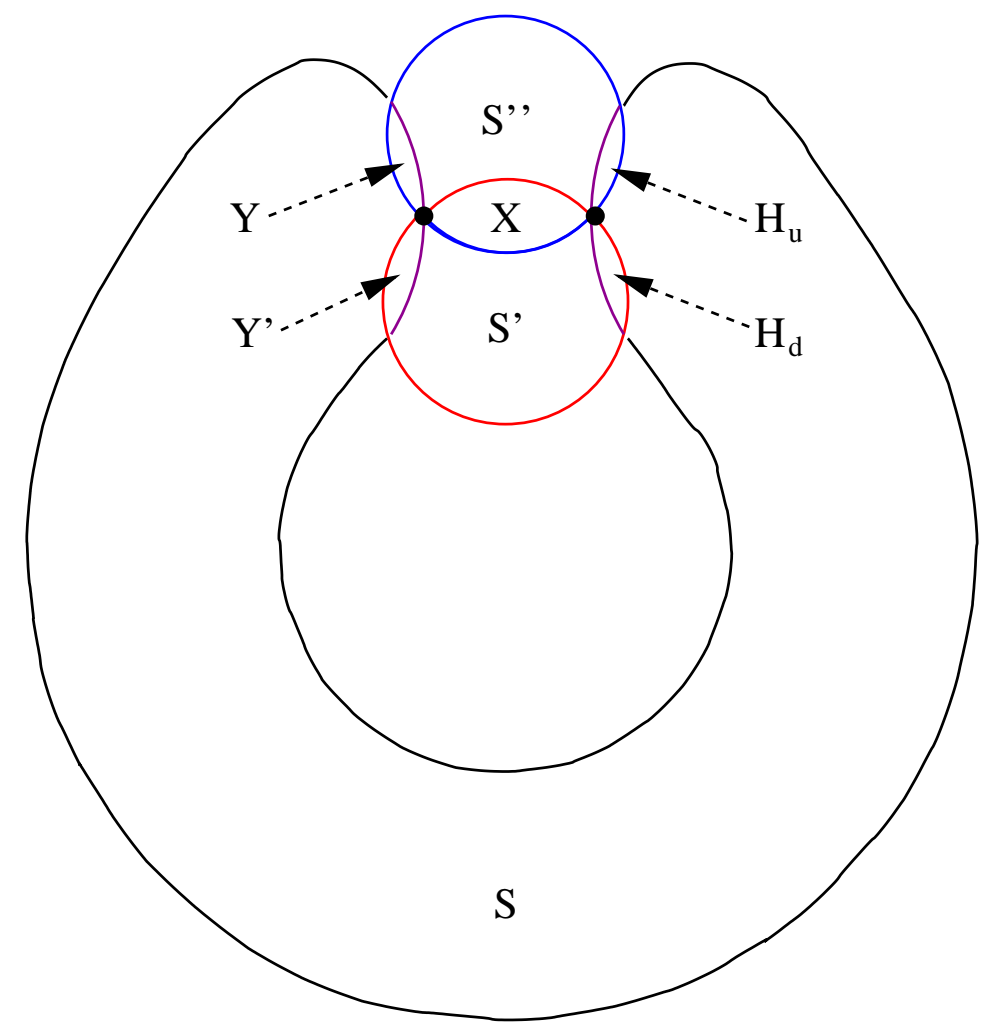

Figure 1: Depiction of the diamond ring model. The GUT model seven-brane wraps the Kähler surface $S$, while the $X$ field localizes at the intersection of two additional seven-branes wrapping the surfaces $S^{\prime}$ and $S^{\prime \prime}$. 
where as explained in section 4, the first term originates from integrating out KaluzaKlein modes associated with $X$.

Given the prominent role that the $X$ field Kaluza-Klein modes play in this class of models, it is important to analyze whether integrating out the other KaluzaKlein modes of the other fields play a similar role in the low energy effective action. To this end, let $\widetilde{X}$ denote the Kaluza-Klein modes associated with the $X$ field and similarly let $\widetilde{\Xi}$ denote the Kaluza-Klein modes for the Higgs fields. In this somewhat condensed notation, the $\widetilde{\Xi}$ denote fields are charged under all three gauge group factors of the MSSM. The messenger sector superpotential is:

$$
\begin{aligned}
& W \supset \lambda_{X Y Y^{\prime}}(X+\tilde{X})(Y+\widetilde{Y})\left(Y^{\prime}+\widetilde{Y^{\prime}}\right)+\lambda_{X^{c} Y^{c} Y^{\prime c}} \widetilde{X^{c}} \widetilde{Y^{c}} \widetilde{Y^{\prime c}} \\
& +\lambda_{X^{c} H_{u} H_{d}} \widetilde{X^{c}}\left(H_{u}+\widetilde{\Xi_{u}}\right)\left(H_{d}+\widetilde{\Xi_{d}}\right)+\lambda_{X H_{u}^{c} H_{d}^{c}}(X+\widetilde{X}) \widetilde{\Xi_{u}^{c}} \widetilde{\Xi_{d}^{c}} \\
& +M_{X} \widetilde{X} \widetilde{X^{c}}+M_{u} \widetilde{\Xi_{u}} \widetilde{\Xi_{u}^{c}}+M_{d} \widetilde{\Xi_{d}} \widetilde{\Xi_{d}^{c}}+M_{Y} \widetilde{Y} \widetilde{Y^{c}}+M_{Y^{\prime}} \widetilde{Y^{\prime} Y^{\prime c}} .
\end{aligned}
$$

Typically the wave functions for the Kaluza-Klein modes and the zero modes will differ, so that the evaluation of these wave functions at a point of triple overlap can induce further structure in the superpotential.

The Kaluza-Klein modes $\widetilde{\Xi_{u}^{c}}$ and $\widetilde{\Xi_{d}^{c}}$ have the same gauge quantum numbers as the messenger fields, and it is therefore tempting to economize the field content of the model. However, in gauge mediated scenarios, these fields do not communicate supersymmetry breaking to the MSSM. To see this, note that to leading order, the additional cubic interaction terms of the superpotential can be neglected and we can fully determine the contribution to the soft masses by computing the log of the effective masses of the messenger fields:

$$
\log \left(M_{t o t}\right)=\sum_{i} \log \left(M_{\text {mess }}^{i}\right)
$$

where the sum $i$ runs over the contributions from the various candidate messenger 
fields. Returning to equations (5.4)-(5.6), the mass terms are:

$$
\begin{aligned}
& W_{Y}^{(2)} \supset \frac{1}{2}\left[\begin{array}{ll}
Y & Y^{\prime}
\end{array}\right]\left[\begin{array}{cc}
0 & \lambda_{X Y Y^{\prime}}\langle X\rangle \\
\lambda_{X Y Y^{\prime}}\langle X\rangle & 0
\end{array}\right]\left[\begin{array}{c}
Y \\
Y^{\prime}
\end{array}\right] \\
& +\frac{1}{2}\left[\begin{array}{cccc}
\widetilde{Y} & \widetilde{Y^{\prime}} & \widetilde{Y^{c}} & \widetilde{Y^{\prime c}}
\end{array}\right]\left[\begin{array}{cccc}
0 & \lambda_{X Y Y^{\prime}}\langle X\rangle & M_{Y} & 0 \\
\lambda_{X Y Y^{\prime}}\langle X\rangle & 0 & 0 & M_{Y^{\prime}} \\
M_{Y} & 0 & 0 & 0 \\
0 & M_{Y^{\prime}} & 0 & 0
\end{array}\right]\left[\begin{array}{c}
\tilde{Y} \\
\widetilde{Y^{\prime}} \\
\widetilde{Y^{c}} \\
\widetilde{Y^{\prime c}}
\end{array}\right], \\
& W_{\Xi}^{(2)}=\frac{1}{2}\left[\begin{array}{llll}
\widetilde{\Xi_{u}^{c}} & \widetilde{\Xi_{d}^{c}} & \widetilde{\Xi_{u}} & \widetilde{\Xi_{d}}
\end{array}\right]\left[\begin{array}{cccc}
0 & \lambda_{X H_{u}^{c} H_{d}^{c}}\langle X\rangle & M_{u} & 0 \\
\lambda_{X H_{u}^{c} H_{d}^{c}}\langle X\rangle & 0 & 0 & M_{d} \\
M_{u} & 0 & 0 & 0 \\
0 & M_{d} & 0 & 0
\end{array}\right]\left[\begin{array}{c}
\widetilde{\Xi_{u}^{c}} \\
\widetilde{\Xi_{d}^{c}} \\
\widetilde{\Xi_{u}} \\
\widetilde{\Xi_{d}}
\end{array}\right] .
\end{aligned}
$$

By inspection, the mass matrix which determines $W_{Y}^{(2)}$ decomposes as the block diagonal sum of a $2 \times 2$ matrix, and a $4 \times 4$ matrix. Further, in the choice of basis made above, the location of non-zero entries in this $4 \times 4$ matrix is identical to the $4 \times 4$ mass matrix which determines $W_{\Xi}^{(2)}$. Because the determinant of each $4 \times 4$ matrix is independent of $X$, we conclude that the Higgs field Kaluza-Klein modes cannot play the role of the messenger fields. Hence, this class of models in fact does require a separate messenger sector.

\section{Strong CP and Gauge Mediation}

Up to this point, we have primarily focussed on the magnitude of the vevs for the chiral superfield $X$. The phase of $X$ will also couple to some of the fields of the MSSM. In this section we show that in the generic case where the $U(1)_{P Q}$ gauge theory is anomalous, a linear combination of this phase and a bulk gravity mode can potentially play the role of the QCD axion. We find that when the gauge mediation variant of the Giudice-Masiero mechanism solves the $\mu$ problem, the axion decay 
constant automatically falls within the experimentally allowed range of values.

To set notation, we now review some of the features of axion models which are germane. See [45,46] for general reviews on axion physics, and [47] and references therein for an extensive discussion of axions in string theory. The strong CP problem is the fact that although the $\mathrm{CP}$ violating operator:

$$
\frac{\bar{\theta}}{32 \pi^{2}} \varepsilon^{\mu \nu \rho \sigma} \operatorname{Tr}_{S U(3)} F_{\mu \nu} F_{\rho \sigma}
$$

can in principle contribute to the Standard Model Lagrangian, the effective $\bar{\theta}$ angle must satisfy the constraint $|\bar{\theta}| \lesssim 10^{-10}$ in order to remain in accord with observation $[48,49]$. Here, $\bar{\theta}$ is defined as the net contribution from the bare theta angle and the phase from the determinant of the mass matrix for all fermions charged under $S U(3)_{C}$.

The axion solution to the strong $\mathrm{CP}$ problem promotes $\bar{\theta}$ to a dynamical field $a$ with Lagrangian density:

$$
L_{\text {axion }}=\frac{f_{a}^{2}}{2} \partial_{\mu} a \partial^{\mu} a+\frac{a}{32 \pi^{2}} \varepsilon^{\mu \nu \rho \sigma} \operatorname{Tr}\left(F_{\mu \nu} F_{\rho \sigma}\right)
$$

where in the above $f_{a}$ is defined as the axion decay constant. The crucial point is that QCD instantons will generate an effective potential for $a$ which has a minimum at zero, thus solving the strong CP problem. More explicitly, the effective potential can be approximated using the pion Lagrangian, see for example section 23.6 of [50]:

$$
V_{a x}(a)=m_{\pi}^{2} f_{\pi}^{2}(1-\cos a)
$$

where $f_{\pi} \sim 90 \mathrm{MeV}$ is the pion decay constant and $m_{\pi} \sim 130 \mathrm{MeV}$ is the mass of the pion. The effective mass of the canonically normalized field $f_{a} \cdot a$ is therefore:

$$
m_{a}=\frac{m_{\pi} f_{\pi}}{f_{a}} \sim \frac{10^{16} \mathrm{eV}^{2}}{f_{a}} .
$$

Current bounds on the value of $f_{a}$ only allow a narrow window of available energy scales:

$$
10^{9} \mathrm{GeV}<f_{a}<10^{12} \mathrm{GeV} .
$$


The lower bound is from estimates on supernova cooling and is difficult to evade. The upper bound is somewhat flexible because it assumes a standard cosmology.

In supersymmetric models, the axion is only one real degree of freedom of a complex scalar. The other real degree of freedom in the complex scalar of the corresponding supermultiplet is the saxion which may be viewed as simply another modulus which must develop a suitable mass to avoid cosmological problems. As emphasized in [51], stabilizing the saxion is a potentially more severe issue than attaining the correct axion decay constant. Moreover, it is possible that in some cases $f_{a}$ could be as high as $10^{15} \mathrm{GeV}$ [51]. In keeping with the principle of decoupling, however, we shall defer all issues concerning moduli stabilization to a later stage of analysis. For this reason, we shall assume the conventional upper bound on the axion decay constant.

In string theory models, there are potentially many candidate axion fields because various moduli fields will generically couple to the QCD instanton density. Note, however, that if the compactification scale sets the dynamics of the axion, the resulting axion decay constant will be above the available window defined by line (6.5). In fact, the ubiquity of such axion-like couplings is also potentially problematic for the strong CP problem. As an example, consider a model where the axion field receives contributions to its effective potential from sources other than QCD instanton effects so that:

$$
V_{a x}(a)=V_{Q C D}(a)+V(a) .
$$

A priori, the minima of $V_{Q C D}$ and $V$ are uncorrelated so that the overall minimum of $V_{a x}$ may shift away from zero. To estimate the size of this shift, let $\theta_{0}$ denote the minimum of $V$. Expanding $V_{a x}$ to quadratic order in $a$ therefore yields:

$$
V_{a x}(a)-\left(V_{Q C D}(0)+V\left(\theta_{0}\right)\right)=\frac{1}{2} V_{Q C D}^{\prime \prime}(0) a^{2}+\frac{1}{2} V^{\prime \prime}\left(\theta_{0}\right)\left(a-\theta_{0}\right)^{2}
$$

it follows that the minimum of $V_{a x}$ shifts to:

$$
\theta=\frac{V^{\prime \prime}\left(\theta_{0}\right)}{V_{Q C D}^{\prime \prime}(0)+V^{\prime \prime}\left(\theta_{0}\right)} \cdot \theta_{0} .
$$


Assuming $\theta_{0}$ is an order one constant, this implies:

$$
\frac{V^{\prime \prime}\left(\theta_{0}\right)}{V_{Q C D}^{\prime \prime}(0)}<10^{-10}
$$

in order for $a$ to solve the strong CP problem.

We now identify possible axion fields in the present class of compactifications. Independent of the details of the particular GUT model seven-brane theory, the QCD instanton density will always couple to the background four-form potential through the seven-brane worldvolume coupling $C_{(4)} \wedge \operatorname{Tr}_{S U(3)}(F \wedge F)$. Letting $c_{S}$ denote the integral of $C_{(4)}$ over the cycle wrapped by $S$, the effective action for $c_{S}$ is:

$$
L_{c}=\frac{M_{*}^{8} \operatorname{Vol}\left(B_{3}\right)}{2} \partial_{\mu} c_{S} \partial^{\mu} c_{S}+\frac{c_{S}}{32 \pi^{2}} \varepsilon^{\mu \nu \rho \sigma} \operatorname{Tr}\left(F_{\mu \nu} F_{\rho \sigma}\right)
$$

As shown in [2], $M_{*}^{8} \operatorname{Vol}\left(B_{3}\right) \sim M_{p l}^{2}$, which establishes that this field cannot play the role of the QCD axion. This is in accord with the general observations in [47] where the most natural axion fields one would think of in the context of string theory end up having too large a decay constant to be phenomenologically viable to be identified with the QCD axion. In fact, as we now argue, a specific linear combination of $c_{S}$ and the phase of the scalar component for the $X$ field can play the role of the QCD axion, thus providing a solution to this problem in the context of string theory. Note that here, the axino is the goldstino mode which is eaten by the gravitino 6

Returning to equations (3.15)-(3.16), note that whereas the contribution to the soft scalar masses only involves $|X|^{2}$, the coupling between $X$ and the gauge field strength kinetic terms $\operatorname{Tr} W^{\alpha} W_{\alpha}$ also includes a coupling to the phase of $X$. With notation as before so that $\langle X\rangle=x+\theta^{2} F$, we shall denote the phase of the vev $x$ by the $2 \pi$ periodic variable $a_{x}$ so that:

$$
x=|x| \exp \left(i a_{x}\right)
$$

\footnotetext{
${ }^{6}$ For earlier discussion on potential connections between the axion and the gravitino, see for example $[52,53]$, and for other discussions on the connection between supersymmetry breaking and the axion, see for example [54].
} 
Expanding equations (3.15)-(3.16) yields:

$$
\begin{aligned}
L & \supset \int d^{4} \theta\left(\sum_{i=1}^{3}-\alpha_{i}^{2} C_{2}\left(R_{\Psi}^{i}\right)\left(\log |X|^{2}\right)^{2} \Psi^{\dagger} \Psi\right) \\
& +\int d^{2} \theta \operatorname{Re}\left(\sum_{i=1}^{3} \frac{1}{8 \pi i}\left(\tau_{Y M}^{(i)}+\frac{1}{2 \pi i} \log X\right) \operatorname{Tr}_{G_{i}} W_{(i)}^{\alpha} W_{(i) \alpha}\right) \\
& =\int d^{4} \theta\left(\sum_{i=1}^{3}-\alpha_{i}^{2} C_{2}\left(R_{\Psi}^{i}\right)\left(\log |x|^{2}+\theta^{2} \frac{F}{x}+\bar{\theta}^{2} \frac{\bar{F}}{\bar{x}}\right)^{2} \Psi^{\dagger} \Psi\right) \\
& +\int d^{2} \theta \operatorname{Re}\left(\sum_{i=1}^{3} \frac{1}{8 \pi i}\left(\tau_{Y M}^{(i)}+\frac{1}{2 \pi i}\left[\log |x|+i a_{x}+\theta^{2} \frac{F}{x}\right]\right) \operatorname{Tr}_{G_{i}} W_{(i)}^{\alpha} W_{(i) \alpha}\right)
\end{aligned}
$$

By inspection, the angle $a_{x}$ couples to the QCD instanton density, as required for a candidate axion.

As we have seen in previous sections, $X$ will generically be charged under at least one $U(1)$ group which must be gauged in a quantum theory of gravity. For example, in the diamond ring model, $X$ transforms in the bifundamental of a $U(1) \times U(1)$ gauge group defined by two intersecting seven-branes. When these $U(1)$ factors are non-anomalous, the vev of $X$ will spontaneously break the gauge symmetry, and the phase of $X$ will be eaten by the gauge boson, eliminating $a_{x}$ as a candidate axion. Alternatively, when these $U(1)$ factors are anomalous, the corresponding gauge bosons will develop a mass via the Green-Schwarz mechanism, leaving behind global symmetries which may potentially be violated by instanton effects. The vev of $X$ spontaneously breaks this symmetry and the corresponding Goldstone mode will persist as a candidate axion 7 We note that independent of any discussion of supersymmetry breaking, the idea that such low energy global symmetries could lead to lower values for the axion decay constant in string based models was already noted in [55]. See section 5 of [47] for a review of the potential role of anomalous $U(1)$ factors in string-motivated axion physics.

Having identified two fields which possess axion-like couplings to the QCD in-

\footnotetext{
${ }^{7}$ More precisely, the Goldstone mode is given by a linear combination of the phase of $X$, with a small contribution from another axion-like field which enters in the Green-Schwarz mechanism.
} 
stanton density, the axion Lagrangian is given by:

$$
\begin{aligned}
L_{a x} & =|x|^{2} \partial_{\mu} a_{x} \partial^{\mu} a_{x}+\frac{1}{2} M_{*}^{8} \operatorname{Vol}\left(B_{3}\right) \partial_{\mu} c \partial^{\mu} c+\frac{a_{x}+c}{32 \pi^{2}} \varepsilon^{\mu \nu \rho \sigma} \operatorname{Tr}\left(F_{\mu \nu} F_{\rho \sigma}\right) \\
& \equiv \frac{f_{a}^{2}}{2} \partial_{\mu}\left(a_{x}+c\right) \partial^{\mu}\left(a_{x}+c\right)+\frac{f_{\perp}^{2}}{2} \partial_{\mu}\left(a_{x}+\beta c\right) \partial^{\mu}\left(a_{x}+\beta c\right) \\
& +\frac{\left(a_{x}+c\right)}{32 \pi^{2}} \varepsilon^{\mu \nu \rho \sigma} \operatorname{Tr}\left(F_{\mu \nu} F_{\rho \sigma}\right)
\end{aligned}
$$

where the linear combination $a_{x}+c$ defines the candidate axion field, and $a_{x}+\beta c$ is an orthogonal linear combination of fields. In the above, the Planck scale enters as $M_{p l}^{2}=M_{*}^{8} \operatorname{Vol}\left(B_{3}\right)$. Solving for the axion decay constant $f_{a}$ yields:

$$
f_{a}=\frac{\sqrt{2} M_{p l}|x|}{\sqrt{M_{p l}^{2}+2|x|^{2}}}=\sqrt{2}|x|+O\left(\frac{|x|^{2}}{M_{p l}^{2}}\right)
$$

where in the final line we have expanded to leading order in the parameter $x$ which is generically smaller than the Planck scale.

Equation (6.19) has important consequences for the parameters of the low energy theory. The effective $\mu$ term, messenger mass and axion decay constant are given by the relations:

$$
\begin{aligned}
\mu_{e f f} & =\gamma \cdot \frac{\bar{F}}{M_{X}} \\
M_{m e s s} & =\lambda_{\text {mess }}|x| \\
f_{a} & =\sqrt{2}|x| \\
\Lambda & =\frac{F}{x} \sim 10^{5} \mathrm{GeV} .
\end{aligned}
$$

This yields the intriguing relation:

$$
f_{a}=\sqrt{2} \frac{M_{X}}{\gamma} \cdot \frac{\mu_{e f f}}{\Lambda}
$$

which connects the GUT scale and weak scale to both the axion decay constant and the scale of supersymmetry breaking. We note that this same relation persists when the number of messenger fields is greater than one. 
To estimate the value of the axion decay constant, we use the same representative values obtained near equation (4.26) so that $\gamma \sim 10, \mu_{\text {eff }} \sim 300 \mathrm{GeV}$ and $M_{X} \sim$ $10^{15.5}$. Setting $\Lambda \sim 10^{5} \mathrm{GeV}$, the resulting value of $f_{a}$ is then:

$$
f_{a} \sim 10^{12} \mathrm{GeV}
$$

Remarkably, this is within the axion window of line (6.5)! Given the fact that this is only an order of magnitude estimate, we find it encouraging that with at most a mild fine-tuning of parameters, connecting weak scale phenomenology to supersymmetry breaking in F-theory automatically produces a viable value for the axion decay constant!

\section{$7 \quad E_{6}, U(1)_{P Q}$ and F-theory}

In previous sections we have shown that when the curve $\Sigma_{X}$ supporting $X$ forms one triple intersection with the Higgs curves and another triple intersection with the messenger curves, interactions with the Kaluza-Klein modes of the $X$ field generate a value for the $\mu$ term in gauge mediated scenarios which is strikingly close to the weak scale. From the perspective of F-theory, however, the identification of the $U(1)_{P Q}$ symmetry appears somewhat accidental. In terms of the particular geometric realization of these matter curves, this translates into the fact that the $\Sigma_{X}$ curve defined by the intersection of two external surfaces $S^{\prime}$ and $S^{\prime \prime}$ must intersect $S$ at two distinct points. While this can certainly be arranged for certain geometries, it appears ad hoc.

These problems can be viewed as symptoms of the fact that the GUT model can undergo a further unification to a higher E-type GUT group. Indeed, perhaps one of the most compelling features of GUT models is the elegant packaging of the representation content of the MSSM into three generations of the $\overline{5} \oplus 10$ of $S U(5)$, and the even further unification to the 16 of $S O(10)$ once right-handed neutrinos are included. Aside from any theoretical bias in favor of this aesthetically appealing structure, the qualitative expectation that the seesaw mechanism can naturally generate small neutrino masses in the Standard Model is at the very least intriguing circumstantial evidence that this type of structure is quite natural for phenomenology as well. In fact, unifying the Higgs and chiral matter into $E_{6}$ allows a further 
unification into the 27 of $E_{6}$.

Each additional stage of unification equips the low energy effective theory with additional $U(1)$ symmetries which are of interest phenomenologically. For example, $S O(10)$ unification contains an additional $U(1)_{B-L}$ factor which can increase the lifetime of the proton. Matter parity can be viewed as a discrete $\mathbb{Z}_{2}$ subgroup of $U(1)_{B-L}$ so that a chiral generation of the MSSM organizes into a copy of the 16 of $S O(10)$ and has parity -1 , while the Higgs fields of the MSSM which descend from the 10 of $S O(10)$ have parity +1 .

Further unification to $E_{6}$ also endows the low energy theory with a $U(1)_{P Q}$ symmetry in the low energy effective theory. To see how this comes about, consider the decomposition of the 78 and 27 of $E_{6}$ into representations of $S O(10) \times U(1)$ :

$$
\begin{aligned}
& E_{6} \supset S O(10) \times U(1) \\
& 78 \rightarrow 45_{0}+1_{0}+16_{-3}+\overline{16}_{+3} \\
& 27 \rightarrow 1_{4}+10_{-2}+16_{1} .
\end{aligned}
$$

In traditional four-dimensional GUT models, it is common to organize all of the matter content of the MSSM into copies of the 27. It is immediate that the additional $U(1)$ charge assignment is consistent with that associated to the $U(1)_{P Q}$ charge for the Higgs fields and chiral matter content of the MSSM. Moreover note that matter parity can be viewed as a discrete $\mathbb{Z}_{2}$ subgroup of this additional $U(1)_{P Q}$.

While traditional four-dimensional GUT models focus on the role of the 27 of $E_{6}$, the $\overline{27}$ plays an equally important role for the mediation sector of the model. Indeed, the $X$ field and messengers can both descend from the $\overline{27}$ of $E_{6}$ upon making the identifications:

$$
\begin{gathered}
X: 1_{-4} \in \overline{27} \\
Y, Y^{\prime}: 1_{+2} \in \overline{27 .} .
\end{gathered}
$$

By inspection, these charge assignments allow an interaction term between $X$ and the messengers, while forbidding a similar interaction term with the Higgs fields, exactly as in the charge assignments we have been assuming in the context of the diamond ring model!

As a brief aside, we recall that in $[1,2]$, our minimal realizations of GUT models 
were based on geometries where the matter content of the MSSM descends from the 78 of $E_{6}$. While it is certainly possible to assign a $U(1)_{P Q}$ charge consistent with the $78^{3}$ interaction term, note that in this intermediate decomposition, the Higgs field descends from the $\overline{16}$ of $S O(10)$. On the other hand, continuing with the natural progression of $E$-type groups, it is also quite natural to package the matter content of the MSSM in terms of the 27.

Perhaps unfortunately, there are also well-known problems in four-dimensional GUT models based on the gauge group $G_{S}=E_{6}$. For example, although the Higgs fields and chiral matter naturally package into the 27 of $E_{6}$, there are three generations of chiral matter, but only one "generation" of Higgs fields. To remove the extraneous Higgs fields from the other two generations of 27's it is necessary to include either higher dimensional representations of $E_{6}$, or higher dimension operators. This additional complexity throws into question the economy of $E_{6}$ as a GUT group.

This issue is in fact more severe in models based on local del Pezzo compactifications of F-theory. As explained in [2], GUT group breaking via fluxes will typically generate additional exotic fields in the zero mode spectrum unless the bulk gauge group is $G_{S}=S U(5)$, or $S O(10)$ when the model descends to a flipped $S U(5)$ model in four dimensions. Although [2] does not contain a complete proof that direct breaking from $G_{S}=E_{6}$ to $G_{\text {std }}$ will always generate exotics, the number of independent instanton configurations is generically a smaller number than the number of different exotic representations which must be excluded from the low energy spectrum. In a certain sense, this is in fact a welcome restriction on the structure of the low energy theory, but appears to clash with the elegant packaging of the matter fields into representations of $S O(10)$ or $E_{6}$.

There is an important loophole to the above considerations which demonstrates the flexibility of local models in F-theory. Recall that in F-theory, a matter curve is defined as a complex codimension one subspace in $S$ where the singularity type enhances by at least one rank from $G_{S}$ to $G_{\Sigma}$ such that $G_{S} \varsubsetneqq G_{\Sigma}$. The matter content localized along the matter curve can be viewed as the intersection of two seven-branes with bulk gauge groups $G_{S}$ and $G_{S^{\prime}}$ such that $G_{S} \times G_{S^{\prime}} \subset G_{\Sigma}$. Many of the examples in $[1,2]$ focussed on rank one enhancement configurations where $G_{S^{\prime}}=U(1)$ or $S U(2)$ because this is the minimal allowed singularity enhancement in F-theory. More generally, however, the singularity type can enhance by more than one rank. 
In order to retain the appealing rigidity of local GUT models found in [2], we shall focus exclusively on local del Pezzo models where the bulk gauge group $G_{S}=S U(5)$ breaks to the Standard Model gauge group via an internal $U(1)$ hyperflux. The matter content of the GUT model can exhibit further unification along curves when the rank of the singularity type increases by more than the minimal required amount. To see how this unification works in practice, first recall that six-dimensional hypermultiplets in the 5 or 10 of $S U(5)$ originate from curves where the bulk singularity $G_{S}=S U(5)$ respectively enhances to $S U(6)$ or $S O(10)$. On the other hand, when $G_{S}=S O(10)$ enhances to $E_{6}$ along a matter curve, a six-dimensional field in the 16 of $S O(10)$ will localize along the same matter curve. Because the 16 of $S O(10)$ unifies the $\overline{5}$ and 10 of $S U(5)$, our expectation is that a local enhancement from $S U(5)$ directly to $E_{6}$ corresponds to a six-dimensional field in the $1+\overline{5}+10$ localized on this curve. To establish this result, we can decompose the adjoint representation of $E_{6}$ to $E_{5} \times U(1)_{2}=S O(10) \times U(1)_{2}$, and then further decompose to irreducible representations of $E_{4} \times U(1)_{1} \times U(1)_{2}=S U(5) \times U(1)_{1} \times U(1)_{2}$. Following the general philosophy of [56], the matter fields which localize on the curve of $E_{6}$ enhancement must be simultaneously charged under these two $U(1)$ subgroup factors. In particular, it follows that a six-dimensional hypermultiplet in the $1_{-5,-3}+\overline{5}_{3,-3}+10_{-1,-3}$ localizes on this matter curve. A similar analysis establishes that a six-dimensional hypermultiplet with matter content specified by a 27 of $E_{6}$ localizes on a curve where the singularity type enhances to $E_{7}$.

This same logic also holds for higher rank enhancements at points of $S$. As in [1], we can start from the adjoint representation of this higher singularity type, and by decomposing the matter fields along each curve which is neutral under a particular subset of generators, we find the expected enhancement in singularity type. For example, we can consider a geometry where $S U(5)$ enhances up to $E_{8}$ at a point of $S$ and such that $E_{8}$ only decreases by one rank along the various matter curves of the geometry. In this case, decomposing the adjoint representation of $E_{8}$ to $E_{6} \times U(1)_{1} \times U(1)_{2}$ yields:

$$
\begin{aligned}
E_{8} & \supset E_{6} \times U(1)_{1} \times U(1)_{2} \\
248 & \rightarrow 78_{0,0}+1_{0,0}+27_{2,0}+27_{-1,-1}+27_{1,-1}+\overline{27}_{-2,0}+\overline{27}_{1,1}+\overline{27}_{-1,1},
\end{aligned}
$$

which implies that there are three curves where the singularity type enhances to $E_{7}$. Further, the low energy theory contains a $27^{3}$ interaction term, where each 27 


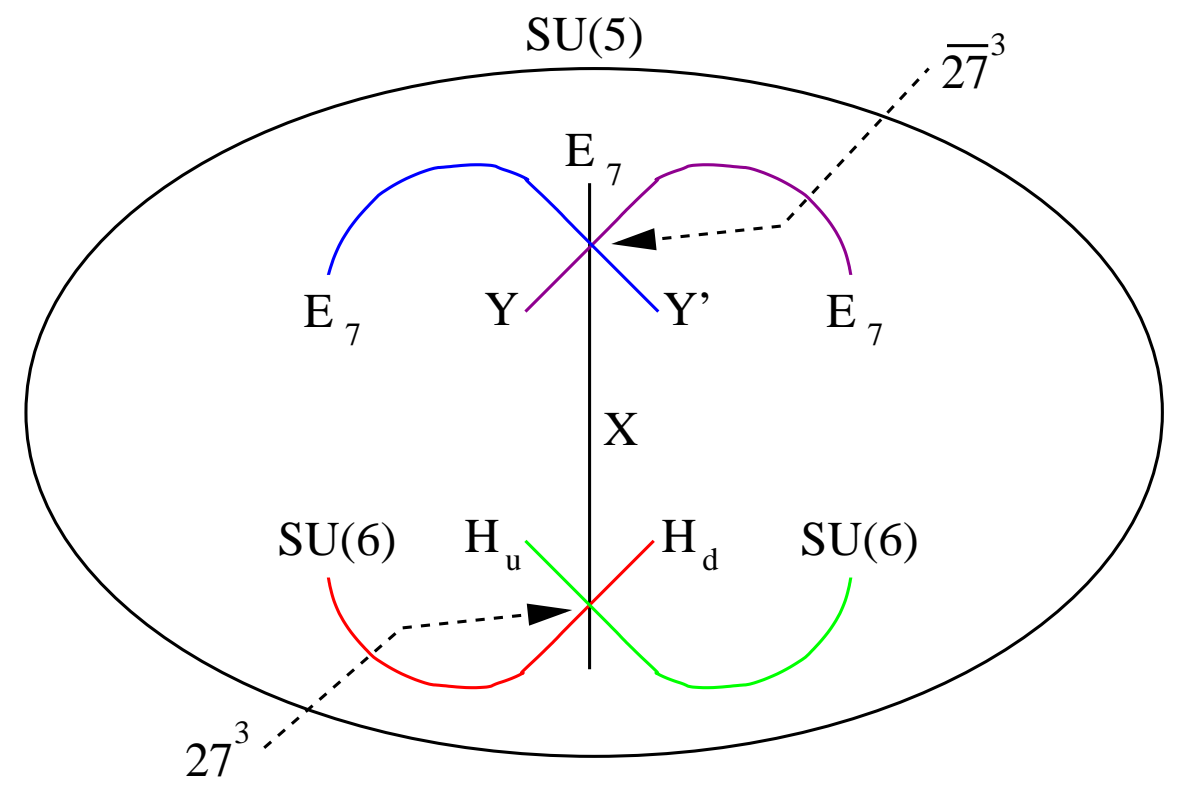

Figure 2: Depiction of the messenger sector of a local $S U(5)$ model where the messengers and $X$ field originate from local enhancements to $E_{7}$ and embed in the $\overline{27}$ of $E_{6}$. The $X Y Y^{\prime}$ interaction term descends from a local enhancement to $E_{8}$ at a point of triple intersection. The Higgs fields originate from local enhancement to $S U(6)$. In this case, the Higgs fields embed in the 27 of $E_{6}$ and can therefore only participate in a $27^{3}$ interaction so that a direct coupling with the $X$ field via the superpotential is forbidden, but an interaction term via the Kähler potential is allowed.

corresponds to a local enhancement in $S U(5)$ to $E_{7}$. It is now immediate that there are many further combinations of rank enhancement which are potentially of interest. As usual, our guiding principal to determine the matter content and allowed interaction terms relies heavily on the close connection between the various ways of partially Higgsing the singularity type at various subspaces of additional enhancement, and the corresponding deformation theory of the geometry. See figure 2 for a depiction of how various $S U(5)$ enhancements to $E_{7}$ can potentially accommodate the interaction terms of the messenger sector.

There is a subtlety in reading off the matter content in the context of higher rank enhancements which we now explain. In the above enhancement from $S U(5)$ to $E_{7}$, we have mainly concentrated on how the 27 obtained from the last step of 
enhancement from $E_{6}$ to $E_{7}$ decomposes into representations of $S U(5)$. In fact, there are additional contributions from the adjoint of $E_{6}$ which come from Higgsing $E_{6}$ down to $S U(5)$. If these additional matter fields localize on the same curve as the 27 , as would be true in the simplest such setup, then we would not be able to control the zero mode content of these contributions independently by adjusting fluxes on the curves. We would simply have too many constraints to satisfy with too few fluxes to choose from. One way to avoid these extra matter fields is to require that they develop a vev, partially Higgsing the corresponding gauge group. In F-theory, this corresponds to brane recombination [1]. It is also possible to consider geometries where a surface of general type locally behaves as a rigid divisor, effectively Higgsing the theory to a local del Pezzo model. Some examples of this type of phenomenon for rigid $\mathbb{P}^{1}$ 's in a local Calabi-Yau threefold can be found in $[57,58]$. In the context of F-theory models, the matter content localized on a curve in the original theory could therefore simply descend to the local del Pezzo model. Finally, it is also possible that an appropriately engineered effective field theory could generate a GUT scale mass for these additional states, removing them from the low energy spectrum. At any rate, we will not address this issue further in this paper and leave a more detailed analysis to future work. Instead, we will focus on the interplay between the matter content of such theories with the PQ symmetry provided by higher rank enhancements in the local singularity type.

In general, when the rank of the singularity enhances by more than one rank, the adjoint representation will contain more than one distinct representation charged under the bulk gauge group $G_{S}$ so that distinct irreducible representations of $G_{S}$ may localize on the same curve. In local models where $U(1)_{P Q}$ embeds in $E_{6}$, the zero mode content along such curves will typically automatically include not just a generation of the MSSM, but also additional $X$ fields, messengers or Higgs fields depending on the overall bundle assignment.

As an explicit example of these considerations, consider the matter content of a curve where the singularity type enhances from $S U(5)$ to $E_{7}$ so that a six-dimensional hypermultiplet in the $27_{1}$ in the $E_{6} \times U(1)$ localizes along this curve. Decomposing $E_{6}$ to the subgroup $S O(10) \times U(1)_{P Q}$, the $27_{1}$ decomposes as:

$$
\begin{aligned}
E_{6} \times U(1) & \supset S O(10) \times U(1)_{P Q} \times U(1) \\
27_{1} & \rightarrow 1_{4,1}+10_{-2,1}+16_{1,1} .
\end{aligned}
$$


It thus follows that the bundle assignments for each representation are:

$$
\begin{aligned}
1_{4,1} & \in K_{\Sigma}^{1 / 2} \otimes L_{P Q}^{4} \otimes L^{1} \\
10_{-2,1} & \in K_{\Sigma}^{1 / 2} \otimes L_{P Q}^{-2} \otimes L^{1} \\
16_{1,1} & \in K_{\Sigma}^{1 / 2} \otimes L_{P Q}^{1} \otimes L^{1}
\end{aligned}
$$

with similar bundle assignments for the complex conjuagate representations. To avoid any subtleties having to do with non-trivial holonomies, we now restrict our analysis to the case where $\Sigma$ is a genus zero curve. Assuming genericity of all bundles, a similar argument will hold in the more general case by analyzing the degrees of the various bundles. The number of fields in the $1_{4,1}$ is completely determined by the number of zero modes in the $10_{-2,1}$ and $16_{1,1}$. Letting $N$ with an appropriate subscript denote the number of fields of a given representation, the bundle assignments for the $10_{-2,1}$ and $16_{1,1}$ are:

$$
\begin{aligned}
10_{-2,1} & \in K_{\Sigma}^{1 / 2} \otimes \mathcal{O}_{\Sigma}\left(N_{10_{-2,1}}\right) \\
16_{1,1} & \in K_{\Sigma}^{1 / 2} \otimes \mathcal{O}_{\Sigma}\left(N_{16_{1,1}}\right) .
\end{aligned}
$$

Solving for the bundles $L_{P Q}$ and $L$ yields:

$$
\begin{aligned}
L & =\mathcal{O}_{\Sigma}\left(2 N_{16_{1,1}}+N_{10_{-2,1}}\right)^{1 / 3} \\
L_{P Q} & =\mathcal{O}_{\Sigma}\left(N_{16_{1,1}}-N_{10_{-2,1}}\right)^{1 / 3}
\end{aligned}
$$

so that the bundle assignment for fields in the $1_{4,1}$ is:

$$
1_{4,1} \in K_{\Sigma}^{1 / 2} \otimes \mathcal{O}_{\Sigma}\left(2 N_{16_{1,1}}-N_{10_{-2,1}}\right)
$$

In other words, the net matter content on this curve is:

$$
R_{n e t}=\left(2 N_{16_{1,1}}-N_{10_{-2,1}}\right) \times 1_{4,1}+N_{10_{-2,1}} \times 10_{-2,1}+N_{16_{1,1}} \times 16_{1,1}
$$

where a negative multiplicity factor indicates that the resulting fields transform in the complex conjugate representation.

The above result demonstrates that when a full generation of the MSSM transforming in the 16 of $S O(10)$ localizes on a matter curve where $S U(5)$ enhances to $E_{7}$, 
the zero mode content of the theory will generically contain additional matter fields. Of particular relevance are matter curves where $N_{16_{1,1}}>0$ and $N_{16_{1,1}}=0$. First consider the case where a matter curve contains at least one chiral generation of the MSSM. In order to solve the doublet triplet splitting problem using an internal $U(1)$ hyperflux, it follows that none of the Higgs fields can also localize on the same curve so that $N_{10_{-2,1}}<0$. Returning to equation (7.18), this implies that such matter curves also support $2\left|N_{16_{1,1}}\right|+\left|N_{10_{-2,1}}\right|$ zero mode GUT group singlets with $U(1)_{P Q}$ charge opposite to that of the $X$ fields. These singlets are essentially harmless in the low energy theory so long as they do not develop a vev, which can typically be arranged. Next consider matter curves where $N_{16_{1,1}}=0$. In this case, equation (17.18) implies that candidate Higgs fields and $X$ fields generically localize on the same matter curve. In particular, the messenger fields do not appear to localize on the same curve as the $X$ field zero modes.

On the other hand, in order to retain the doublet triplet splitting mechanism via hyperflux proposed in [2], the Higgs fields must localize on a curve of $S U(6)$ enhancement. Indeed, when additional matter localizes on a curve, the $U(1)$ hyperflux will generate additional zero modes. Assuming that the Higgs field localizes on a curve where the singularity type enhances to $S U(6)$, the requisite interaction term between the Higgs field and chiral matter of the MSSM now requires that a local $S U(6)$ enhancement must form a triple intersection with two curves which enhance to $E_{7}$. Note that if such an intersection can be realized, the Higgs fields will automatically come equipped with the correct $U(1) \mathrm{PQ}$ charge.

We now present a local construction of such an enhancement. At the point of triple intersection, the singularity type must necessarily enhance to $E_{8}$. The bulk $S U(5)$ gauge group can therefore be viewed as one of the factors in the decomposition of $E_{8}$ to $S U(5) \times S U(5)$. With respect to this subgroup, the adjoint representation of $E_{8}$ decomposes as:

$$
\begin{aligned}
E_{8} & \supset S U(5)_{1} \times S U(5)_{2} \\
248 & \rightarrow(24,1)+(1,24)+(5,10)+(\overline{5}, \overline{10})+(10, \overline{5})+(\overline{10}, 5)
\end{aligned}
$$

where we shall take the bulk gauge group to correspond to the $S U(5)_{1}$ factor. A local enhancement in singularity type of the $S U(5)_{1}$ corresponds to the locus where some directions in the Cartan of $S U(5)_{2}$ combine with $S U(5)_{1}$ to form a higher rank 
singularity. To set notation, let $t_{1}, \ldots, t_{5}$ denote the local generators of the Cartan of $S U(5)_{2}$ subject to the tracelessness condition $t_{1}+\ldots+t_{5}=0$. Additional massless states of $\mathrm{SU}(5)_{2}$ will contribute along various directions in the Cartan subalgebra. Some examples of rank one enhancements can be achieved when the 5 or 10 of $S U(5)_{2}$ contribute to the massless states of $S U(5)_{1}$. Indeed, the 5 contributes when $t_{i}=0$, while the 10 contributes when $t_{i}+t_{j}=0$ for $i \neq j$, corresponding to the local enhancements:

$$
\begin{aligned}
& S O(10): t_{i}=0 \\
& S U(6): t_{i}+t_{j}=0 .
\end{aligned}
$$

Along $t_{i}=0$, we find $10 \times 2+1=21$ additional states contribute so that the 24 of $S U(5)$ enhances to the 45 of $S O(10)$. Moreover, along $t_{i}+t_{j}=0$, we find $5 \times 2+1=11$ additional states so that the adjoint of $S U(5)$ instead enhances to the adjoint of $S U(6)$. A local enhancement to $E_{7}$ corresponds to the direction in the Cartan of $S U(5)_{2}$ where an entire $S U(3) \times U(1)$ subgroup of $S U(5)_{2}$ combines with $S U(5)_{1}$. In terms of the local generators of the Cartan of $S U(5)_{2}$ this reads:

$$
\begin{aligned}
& E_{7}: t_{1}+t_{2}=t_{3}=t_{4}=t_{5}=0 \\
& E_{7}^{\prime}: t_{4}+t_{5}=t_{1}=t_{2}=t_{3}=0
\end{aligned}
$$

Decomposing various representations of $S U(5)_{2}$ into irreducible representations of the maximal subgroup $S U(3) \times S U(2) \times U(1)$ yields:

$$
\begin{aligned}
S U(5)_{2} & \supset S U(3) \times S U(2) \times U(1) \\
24 & \rightarrow(8,1)_{0}+(1,3)_{0}+(3, \overline{2})_{-5}+(\overline{3}, 2)_{+5}+(1,1)_{0} \\
10 & \rightarrow(1,1)_{6}+(3,2)_{1}+(\overline{3}, 1)_{-4} \\
5 & \rightarrow(3,1)_{-2}+(1,2)_{+3} .
\end{aligned}
$$

In particular, we conclude that along the loci defined by lines (7.23) and (7.24), the 5 contributes three states $\left(t_{i}=0\right.$ for $\left.i \geq 3\right)$, the 10 contributes four states $\left(t_{i}+t_{j}=0\right.$ for $i, j \geq 3$ and $\left.i \neq j\right)$, and the 24 contributes nine states for a total of $2 \times 10 \times 3+2 \times 5 \times 4+9=109$ additional states. Combined with the adjoint of $S U(5)_{1}$, this indeed yields the 133 expected states of the adjoint of $E_{7}$.

To be completely explicit, we now present a local model which exhibits the desired 
enhancement type. To this end, let $z_{1}$ and $z_{2}$ denote two local coordinates in the vicinity of the local $E_{8}$ enhancement at the point $z_{1}=z_{2}=0$. In terms of these coordinates, one realization of the desired triple intersection is:

$$
\begin{aligned}
& t_{1}=z_{2}\left(z_{1}+z_{2}\right) \\
& t_{2}=z_{2}\left(z_{1}-z_{2}\right) \\
& t_{3}=-4 z_{1} z_{2} \\
& t_{4}=z_{1}\left(z_{1}+z_{2}\right) \\
& t_{5}=z_{1}\left(-z_{1}+z_{2}\right) .
\end{aligned}
$$

Along the locus $z_{1}=0$, we note that $t_{3}=t_{4}=t_{5}=0$, and $t_{1}+t_{2}=0$, which corresponds to a local enhancement of $S U(5)$ to $E_{7}$. Similarly, along $z_{2}=0$, we obtain another local enhancement from $S U(5)$ to $E_{7}$. There are also several curves along which $S U(5)$ enhances to $S U(6)$. For example, $t_{1}+t_{3}=z_{2}\left(z_{2}-3 z_{1}\right)$ vanishes along $z_{2}=0$, and $z_{2}=3 z_{1}$. Along this second locus, the local behavior of the $t_{i}$ 's is:

$$
\begin{aligned}
& t_{1}=12 z_{1}^{2} \\
& t_{2}=-6 z_{1}^{2} \\
& t_{3}=-12 z_{1}^{2} \\
& t_{4}=4 z_{1}^{2} \\
& t_{5}=2 z_{1}^{2} .
\end{aligned}
$$

which establishes that no additional states from a 10 or 5 of $S U(5)_{2}$ beyond those expected become massless. Thus, the required triple intersection of matter curves is realized by the local coordinates:

$$
\begin{aligned}
E_{7}: z_{1} & =0 \\
E_{7}^{\prime}: z_{2} & =0 \\
S U(6) & : z_{2}=3 z_{1} \\
E_{8}: & z_{1}=z_{2}=0,
\end{aligned}
$$

as desired. In this particular case, we note that the local enhancement to $E_{7}$ may contain additional matter fields beyond the 27 of $E_{6}$. In this regard, this example should be viewed as a starting point for a more complete analysis. 


\section{Fayet-Polonyi Model of Supersymmetry Break- ing}

On general grounds, there are likely to be several dynamical mechanisms available which generate a vev for $X$ consistent with gauge mediated supersymmetry breaking. Rather than posit the existence of an entirely new sector which would inevitably dilute the predictive power of the theory, in this section we show that the anomalous $U(1)_{P Q}$ gauge theory which has already figured prominently in this paper will in many cases break supersymmetry. Along these lines, we also show that this sector of the theory can naturally accommodate a low scale of supersymmetry breaking consistent with the other results of this paper.

The anomalous $U(1)_{P Q}$ gauge theory is effectively a combination of a Fayet model D-term potential which sets the value of $x$, and a Polonyi term which sets the value of $F$ in the vev $\langle X\rangle=x+\theta^{2} F$. In this regard, it is important that the $U(1)_{P Q}$ symmetry is both gauged and anomalous. The Fayet-Iliopoulos parameter $\xi_{P Q}$ is determined by the background flux through the Peccei-Quinn seven-brane. By appealing to a variant of the Bousso-Polchinski flux-scanning argument of [59], we show that $x \sim \Delta_{\min } \cdot M_{X}$ where $\Delta_{\min } \sim M_{X} / M_{p l}$ is the minimal flux spacing. The fact that the $U(1)_{P Q}$ symmetry is anomalous is more crucial for the Polonyi term. Indeed, precisely because the $U(1)_{P Q}$ symmetry is anomalous, instanton effects will generate superpotential terms which violate the global $U(1)_{P Q}$ symmetry of the low energy theory.

The rest of this section is organized as follows. First, we spell out in field theory terms what we mean by the Fayet-Polonyi model. Next, we focus on the specific realization of this model in our context. To this end, we determine the D-term potential of the anomalous $U(1)_{P Q}$ theory and further show that instanton effects generate a linear superpotential term which breaks supersymmetry. To complete our analysis, we next show that the Fayet-Polonyi model breaks supersymmetry at a scale consistent with solving both the $\mu$ problem and strong CP problem. Finally, we show how the bosonic partner of the axion, the saxion is stabilized in the present class of models. Some additional more technical discussion of higher order instanton effects and their potential relevance for the axion potential is deferred to Appendix A. 


\subsection{Generalities of Fayet-Polonyi Model}

Here we briefly specify in field theoretic terms what we mean by a Fayet-Polonyi model of SUSY breaking. First we recall each of the two models and then present the hybrid model.

We start with the Fayet model [60] 8 This is a model of an $\mathcal{N}=1$ supersymmetric $U(1)$ gauge theory coupled to some charged fields $X, Y$ with respective charges +1 and -1 . In this model, the FI parameter $\xi$ is non-zero, and the superpotential is given by:

$$
W=m X Y .
$$

The physical potential is a combination of the F-term contribution and the D-term contribution:

$$
V=\left|\frac{\partial W}{\partial X}\right|^{2}+\left|\frac{\partial W}{\partial Y}\right|^{2}+\frac{1}{2} g^{2} D^{2}
$$

where $g$ is the gauge coupling and:

$$
D=|X|^{2}-|Y|^{2}-\xi .
$$

Supersymmetry is preserved only when $\partial W / \partial X=\partial W / \partial Y=D=0$. The F-flatness conditions require the vev of the fields $X, Y$ to be zero. On the other hand, this is incompatible with the vanishing $D$-term which requires one of the two fields $X$ or $Y$ to cancel the contribution from the FI term $\xi$. For large enough $\xi$ (i.e. if $\xi \gg m^{2} / g^{2}$ ) the minimum of the potential will be dictated by screening the D-term. Without loss of generality, consider the case where $\xi$ is large a positive so that $X$ attains a non-zero vev. In this case, the top component of the $Y$ field $F_{Y}=m X \neq 0$ so that supersymmetry is broken. The explicit value of $F_{Y}$ is:

$$
F_{Y}=m \sqrt{\xi-\frac{m^{2}}{g^{2}}} .
$$

Note this mechanism works even if there are more charged fields and that these additional fields typically pick up contributions to their masses on the order of $m$, due to the imperfect screening of the D-term.

\footnotetext{
${ }^{8}$ See [61-63] for early work on supersymmetry breaking which exploits the presence of related $U(1)$ symmetries.
} 
We now turn to the Polonyi model [64]. This is a model of a chiral superfield with a superpotential of the form

$$
W=\kappa X
$$

Since $\kappa=\partial W / \partial X \neq 0$, this model breaks supersymmetry because for a generic interacting field theory where the Kähler potential $K(X, \bar{X})$ is non-trivial, this SUSY breaking cannot be absorbed away by an overall shift in the vacuum energy density.

As its name suggests, the hybrid Fayet-Polonyi model combines elements from both models. Consider again a theory of an $\mathcal{N}=1$ supersymmetric $U(1)$ gauge theory coupled to various charged matter fields. Let $X$ denote one such field. Moreover let us assume that the $U(1)$ is anomalous and is Higged through the Green-Schwarz mechanism, which in particular requires the existence of an FI-term $\xi$. Because the low energy global $U(1)$ symmetry is anomalous, instanton effects will likely generate $U(1)$ violating terms in the superpotential:

$$
W(X)=\kappa X+\ldots
$$

where in particular the linear term $\kappa$ is not zero. Note that $\kappa$ is expected to be very small, if it is an instanton generated effect, of the order of $\Lambda_{0}^{2} \exp \left(-1 / g^{2}\right)$ for some cutoff mass scale $\Lambda_{0}$. In the application we will have for the PQ brane, the higher powers of $X$ could also potentially appear in the superpotential but they are much smaller than the leading term, and so we have effectively a Polonyi-like superpotential. Note that the fact that the superpotential seems to violate $U(1)$ charge is not inconsistent with gauge symmetry, because the instanton corrections, captured by $\kappa$ pick up anomalous $U(1)$ charge exactly to neutralize the term. The same comment applies to the higher monomials as well. The mechanism of supersymmetry breaking is now very similar to that of the Fayet model: The non-vanishing $\xi$ term drives $X$ to have a non-trivial VEV of the order of $\sqrt{\xi-a}$ where $|a| \sim\left(\left|W^{\prime} W^{\prime \prime} / g^{2} X\right|\right)_{|X|=\sqrt{\xi}}$. The term $a$ also sets the scale for the mass contributions from the VEV of $X$ to the other fields charged under this $U(1)$ (due to the imperfect screening of the D-term). In the application below such mass corrections are rather small and do not make significant contributions. Indeed the first contribution to these mass terms will come from the coefficient of $X^{2}$ and if generated by instanton effects will generically be far smaller in scale than the linear term. Although ultimately different, see [65] for a 
related model of supersymmetry breaking which combines elements from the Fayet and Polonyi models.

\subsection{Fayet-Polonyi Model from a $U(1)_{P Q}$ Seven-Brane}

In this subsection we describe the explicit realization of the Fayet-Polonyi model based on a PQ seven-brane. Although the $U(1)_{P Q}$ symmetry can sometimes correspond to a linear combination of distinct $U(1)$ factors on different seven-branes, this is irrelevant for the purposes of the present analysis, and we shall therefore always consider the case where $U(1)_{P Q}$ is realized on a single seven-brane.

Now, as we have seen in previous sections, all of the fields of the MSSM are necessarily charged under $U(1)_{P Q}$. Hence, the $U(1)_{P Q}$ seven-brane theory will contain several different matter curves which will all contribute to the four-dimensional effective theory. The zero mode content of the four-dimensional theory is then determined by an appropriate choice of background flux on the GUT model seven-brane, the $U(1)_{P Q}$ seven-brane, and possibly other seven-branes of the compactification. To set notation, let $\Psi$ denote a generic chiral superfield charged under $U(1)_{P Q}$ which localizes on a curve $\Sigma_{\Psi}$, and $X$ a chiral superfield localized on a curve $\Sigma_{X}$ which can potentially develop a supersymmetry breaking vev.

Before proceeding to the explicit realization of the Fayet-Polonyi model, we now describe some general features of the $U(1)_{P Q}$ gauge theory realized on a sevenbrane. For a generic choice of background fluxes on the $U(1)_{P Q}$ seven-brane, the zero mode content of the theory will be such that the four-dimensional $U(1)_{P Q}$ symmetry is anomalous. As a consequence, the corresponding gauge boson in the fourdimensional effective theory will develop a mass due to the Green-Schwarz mechanism. More explicitly, in the eight-dimensional worldvolume theory of the PQ seven-brane, there is an axion-like coupling between the RR four-form potential and the $U(1)_{P Q}$ gauge field strength of the form:

$$
L_{P Q} \supset M_{*}^{8} \int_{\mathbb{R}^{3,1} \times B_{3}} d C_{(4)} \wedge *_{10} d C_{(4)}+M_{*}^{4} \int_{\mathbb{R}^{3,1} \times S_{P Q}} C_{(4)} \wedge F_{\mathbb{R}^{3,1}} \wedge\left\langle F_{P Q}\right\rangle .
$$

Here, $F_{\mathbb{R}^{3,1}}$ and $F_{P Q}$ respectively denote the $U(1)_{P Q}$ field strengths in the fourdimensional spacetime directions and the Kähler surface $S_{P Q}$ wrapped by the PQ 
seven-brane. Reducing to the four-dimensional effective theory, we note that under a $U(1)_{P Q}$ gauge transformation, the four-form will in general shift by an amount which exactly cancels the contribution from the anomaly. Letting $\mathcal{C}$ denote the four-dimensional superfield associated with the reduction of the four-form $C_{(4)}$ and $V_{P Q}$ the vector multiplet for the $U(1)_{P Q}$ gauge boson so that under the gauge transformation $V_{P Q} \mapsto V_{P Q}+i \Lambda_{P Q}-i \Lambda_{P Q}^{\dagger}, \mathcal{C} \mapsto \mathcal{C}+i \Lambda_{P Q}$, the corresponding action in superspace is:

$$
L_{P Q} \supset \int d^{4} \theta K\left(\mathcal{C}+\mathcal{C}^{\dagger}-V_{P Q}\right)
$$

where $K$ denotes an appropriate Kähler potential. Expanding in powers of $V_{P Q}$, this is given as:

$$
L_{P Q} \supset \int d^{4} \theta K\left(\mathcal{C}+\mathcal{C}^{\dagger}\right)-K^{\prime}\left(\mathcal{C}+\mathcal{C}^{\dagger}\right) V_{P Q}+\frac{1}{2} K^{\prime \prime}\left(\mathcal{C}+\mathcal{C}^{\dagger}\right) V_{P Q}^{2}+\ldots
$$

Fixing a background value of $\mathcal{C}$ as $\mathcal{C}_{0}$ so that $\mathcal{C}=\mathcal{C}_{0}+c$, in terms of component fields, we conclude that the four-dimensional effective action contains the terms:

$$
L_{P Q} \supset \frac{1}{2} \xi_{*}\left(\partial_{\mu} c+A_{\mu}\right)^{2}+\xi_{*} D_{P Q}
$$

where $\sqrt{\xi_{*}}$ is a mass scale associated with the PQ seven-brane, and $D_{P Q}$ denotes the usual auxiliary field of the vector multiplet. In the above, we have dropped irrelevant constant multiplicative factors of order one which will not play any role in the discussion to follow. By inspection, the first term in equation (8.10) will contribute to the mass of the $U(1)_{P Q}$ gauge boson, while the second term contributes to the FI parameter of the four-dimensional theory.

Depending on the particular flux data through the seven-brane theory, there could potentially be additional contributions to the effective action. In particular, in subsection 8.2 .1 we show that there is an additional contribution to the FI parameter from fluxes when the Kähler form is not orthogonal to $\left\langle F_{P Q}\right\rangle$. Moreover, precisely because the $X$ field is charged under the anomalous $U(1)_{P Q}$ symmetry, it follows that the phase of the $X$ field $a_{x}$ studied in section $[6$ as a candidate axion field will also shift under a $U(1)_{P Q}$ gauge transformation. Including the contribution from 
these terms, we have:

$$
L_{P Q} \supset|x|^{2}\left(\partial_{\mu} a_{x}+A_{\mu}\right)^{2}+\frac{1}{2} \xi_{*}\left(\partial_{\mu} c+A_{\mu}\right)^{2}+\left(\xi_{\text {flux }}+\xi_{*}\right) D_{P Q} .
$$

In the following, we shall refer to the net FI parameter as:

$$
\xi_{P Q} \equiv \xi_{f l u x}+\xi_{*}
$$

In general, $\left|\xi_{*}\right| \gg|x|$, so that the mass of the $U(1)_{P Q}$ gauge boson will typically be close to the GUT scale. On the other hand, in subsection 8.2.1 we will show that by scanning over all fluxes, the minimal non-zero value of the mass scale $\sqrt{\xi_{P Q}}$ is typically in the range required for $a_{x}$ to play the role of the QCD axion.

From the perspective of the four-dimensional effective theory, it may at first appear puzzling that $x$ can develop any vev at all due to the D-term potential. Indeed, since the gauge boson is very heavy, it can effectively be integrated out. In this context, there is no D-term potential to speak of. The essential point is that at high energy scales, the D-term potential is more appropriately written as:

$$
V_{D}=2 \pi \alpha_{P Q}\left(|x|^{2}-K^{\prime}\left(\mathcal{C}+\mathcal{C}^{\dagger}\right)+\xi_{\text {flux }}\right)^{2} .
$$

The condition $V_{D}=0$ corresponds to a background field configuration where in general both $x$ and $\mathcal{C}$ develop non-zero vevs. The essential point we will exploit later is that the vev of $x$ can be tuned to be lower than the mass scale of the PQ gauge boson. The actual axion will then be given as a linear combination of the phase of $x$, with a small contribution from $c$ as well. Indeed, this corresponds to a flat direction of the potential $V_{D}$. Besides the axion, the other bosonic component of the corresponding supermultiplet is the saxion. We now show how the D-term and Fterm potentials of the Fayet-Polonyi model can be generated in the PQ seven-brane theory.

\subsubsection{D-Term Potential}

As shown above, the four-dimensional anomalous $U(1)_{P Q}$ gauge boson will develop a mass through a Green-Schwarz mechanism via an axion-like coupling between the RR four-form and the eight-dimensional $U(1)_{P Q}$ field strength. In the four-dimensional 
effective theory, the strength of this coupling is proportional to the net flux $F_{P Q}$ through $S_{P Q}$. The presence of this flux can induce additional contributions to the FI parameters when $F_{P Q}$ is not orthogonal to the Kähler form on $S_{P Q}$. For ease of discussion, we will present this analysis in the simplified case where there is only a single matter curve $\Sigma_{X}$ corresponding to the $X$ field of the $U(1)_{P Q}$ theory.

First recall that the D-term equation of motion for the $U(1)_{P Q}$ seven-brane theory is $[1]$ :

$$
\omega_{P Q} \wedge F_{P Q}=\frac{1}{2} \omega_{P Q} \wedge \delta_{\Sigma_{X}}\left[\mu(\sigma, \bar{\sigma})-\mu\left(\overline{\sigma^{c}}, \sigma^{c}\right)\right]
$$

which holds pointwise on the surface $S_{P Q}$ wrapped by the Peccei-Quinn seven-brane. In the above, $\omega_{P Q}$ denotes the Kähler form on $S_{P Q}$ and $F_{P Q}$ is the internal gauge field strength. In addition, at each point of $\Sigma_{X}, \sigma$ and $\sigma^{c}$ respectively label the scalar component of the four-dimensional $\mathcal{N}=1$ chiral superfields $\mathbb{X}$ and $\mathbb{X}^{c}$ and $\mu$ denotes the natural moment map pairing defined on $\Sigma_{X}[1]$. Expanding about a background gauge field configuration on $\Sigma_{X}$, let $X_{i}$ denote the zero modes of $\mathbb{X}$ with similar conventions for $X_{i_{c}}^{c}$. Integrating equation (8.14) over the curve $\Sigma_{X}$ implies:

$$
\begin{aligned}
\left.\int_{\Sigma_{X}}\left(\omega_{P Q} \wedge F_{P Q}\right)\right|_{\Sigma_{X}} & =\frac{1}{2}\left[\sum_{i} \mu\left(x_{i}, \bar{x}_{i}\right)-\sum_{i_{c}} \mu\left(\bar{x}_{i_{c}}^{c}, x_{i_{c}}^{c}\right)\right] \\
& \sim e_{X} \frac{M_{*}^{2} V \operatorname{Vol}\left(\Sigma_{X}\right)}{2}\left[\sum_{i}\left|x_{i}\right|^{2}-\sum_{i_{c}}\left|x_{i_{c}}^{c}\right|^{2}\right]
\end{aligned}
$$

where $e_{X}$ denotes the integral charge of $X$ under $U(1)_{P Q}$. In the above, we have used the fact that the zero modes are orthogonal in the sense that $\mu\left(x_{i}, \bar{x}_{j}\right)=0$ for $i \neq j$. Canonically normalizing the kinetic terms for $X$ and $X^{c}$, the D-term equation of motion in the four-dimensional effective theory is:

$$
\left.\xi_{\text {flux }} \equiv \int_{\Sigma_{X}}\left(\omega_{P Q} \wedge F_{P Q}\right)\right|_{\Sigma_{X}}=\frac{e_{X}}{2}\left[\sum_{i}\left|x_{i}\right|^{2}-\sum_{i_{c}}\left|x_{i_{c}}^{c}\right|^{2}\right]
$$

where by abuse of notation, we have labelled the rescaled $x$ 's by the same variable. Equation (8.17) demonstrates that the FI parameter of the $U(1)_{P Q}$ gauge theory is given by integrating the background flux over $\Sigma_{X}$. Letting $V_{P Q}$ denote the vector multiplet of the $U(1)_{P Q}$ gauge theory, in four-dimensional $\mathcal{N}=1$ superspace, the 
effective action for the $X$ zero modes contains the terms:

$$
L_{P Q}^{(0)} \supset \int d^{4} \theta\left[\left(X_{i}\right)^{\dagger} e^{t V_{P Q}} X_{i}+\left(X_{i_{c}}^{c}\right)^{\dagger} e^{-t V_{P Q}} X_{i_{c}}^{c}+\frac{2 t}{e_{X}} \xi_{f l u x} V_{P Q}\right]
$$

where $t=e_{X} \cdot g_{P Q} / 2$. Returning to equation (8.12), we conclude that the net FI term is given by the sum of the bulk contribution $\xi_{*}$, and contributions from matter curves, $\xi_{\text {flux }}$.

Having identified the origin of the Fayet-Iliopoulos term in the anomalous $U(1)_{P Q}$ theory, the D-term potential is therefore given by:

$$
V_{\text {Fayet }}=2 \pi \alpha_{P Q} \cdot D_{P Q}^{2}=2 \pi \alpha_{P Q} \cdot\left(|X|^{2}+\sum_{\Psi} e_{\Psi}|\Psi|^{2}-K^{\prime}\left(\mathcal{C}+\mathcal{C}^{\dagger}\right)+\xi_{f l u x}\right)^{2}
$$

where $\alpha_{P Q}$ is the fine structure constant of the $U(1)_{P Q}$ gauge theory, $\xi_{P Q}$ is given by equation (8.12), the $\Psi$ 's denote all fields besides $X$ charged under $U(1)_{P Q}$, and $e_{\Psi}$ denotes the integral charge of $\Psi$ in a normalization where $e_{X}=2$. Here, we have also included the presence of the field $\mathcal{C}$.

\subsubsection{F-Term Potential}

In this subsection we sketch the form of the superpotential generated by Euclidean three-branes wrapping a del Pezzo surface $S_{P Q}$. Instanton effects in type II string theory have recently been investigated, for example, in [66-68]. Particular applications to local F-theory models have been studied in $[27,28]$. For simplicity, we shall assume that instanton effects from Euclidean three-branes wrapping other Kähler surfaces in the geometry are sufficiently small that they can safely be neglected. Instantons will generically contribute to any $U(1)$ seven-brane theory with matter localized on curves. To see why this is so, consider the reduction of the eight-dimensional seven-brane theory to the six-dimensional theory defined by one such matter curve. The net contribution to the six-dimensional gauge anomaly is proportional to $\operatorname{TrF}^{4}$ so that all contributions to the anomaly will contribute with the same sign. Because this symmetry is anomalous, we expect instantons of the six-dimensional theory to contribute to the effective superpotential.

Of course, the particular form of the instanton generated superpotential will 
depend on the zero mode content of the four-dimensional effective theory. The $k$ instanton sector contribution to the superpotential is given by summing over all internal fluxes of $k$ Euclidean three-branes wrapping the surface $S_{P Q}$. Letting $X_{1}, \ldots, X_{p}$ denote the various zero modes of the four-dimensional effective theory, the net contribution from instantons is:

$$
W_{\text {inst }}^{\text {tot }}=\sum_{I, k, f_{\text {inst }, k, m}} c_{I, k, f_{\text {inst }, k, m}} q^{k} \cdot w^{\nu\left(f_{\text {inst }, k, I}\right)} X^{I}
$$

where $I=\left(i_{1}, \ldots, i_{p}\right)$ is a multi-index, and $X^{I} \equiv X_{1}^{i_{1}} \cdots X_{p}^{i_{p}}$. Letting $\tau_{P Q}$ denote the complexified gauge coupling constant of the PQ gauge theory and $\tau_{I I B}$ the axiodilaton, $q=\exp \left(2 \pi i \tau_{P Q}\right)$ is the generic instanton contribution from a three-brane wrapping $S_{P Q}$, and $w=\exp \left(2 \pi i \tau_{I I B}\right)$ is a contribution which depends on the internal instanton number $\nu\left(f_{\text {inst }, k, I}\right)$ through a given three-brane configuration defined by the supersymmetric internal flux $f_{\text {inst }, k, I}$. Here, we have indicated the schematic form of the contribution, because in general F-theory backgrounds, $\tau_{I I B}$ will have non-trivial position dependence. In such cases, the contribution from the analogue of $w$ will correspond to the integration of $\tau_{I I B}$ against the instanton density defined by the internal flux.

The background choice of fluxes can in many cases lead to a Polonyi-like superpotential, and here we shall assume that this is realized in the present class of models. In the related explicit example of [27], it was shown that this is indeed the case for a configuration of seven-branes wrapping appropriate del Pezzo surfaces which intersect along a rigid genus zero matter curve. When a single zero mode $X$ localizes on this curve, instantons of the higher dimensional gauge theory will generate the leading order superpotential terms:

$$
W_{\text {inst }}^{\text {tot }}=M_{P Q}^{2} \kappa_{1} q X+O\left(q^{2}\right)
$$

where the $\kappa_{i}$ correspond to moduli dependent worldvolume determinant factors, and we have also absorbed the contribution from $w$ into $\kappa_{i}$ as well. More generally, for an appropriate choice of background flux through the PQ seven-brane, a similar analysis establishes that this same contribution will also be present even when additional zero modes localize on other curves. Although the other fields of the MSSM are charged under $U(1)_{P Q}$, our expectation is that these instanton effects will be dominated 
by other contributions to the tree level superpotential. Some additional technical details on this point as well as some discussion on higher order instanton corrections are deferred to Appendix $\mathrm{A}$.

Including all terms which involve a non-trivial dependence on $X$, the full superpotential is therefore of the form:

$$
W(X, Y)=\lambda X Y Y^{\prime}+M_{P Q}^{2} \kappa_{1} q X+O\left(M_{P Q} q^{2}\right)+O(q)
$$

In the above, the $O\left(M_{P Q} q^{2}\right)$ term corresponds to possible multi-instanton contributions involving only the $X$ field, and the $O(q)$ term refers to possible contributions involving MSSM fields. At leading order, the F-term potential is therefore:

$$
\begin{aligned}
V_{\text {Polonyi }} & =\left|\frac{\partial W(X, Y)}{\partial X}\right|^{2}+\left|\frac{\partial W(X, Y)}{\partial Y}\right|^{2}+\left|\frac{\partial W(X, Y)}{\partial Y^{\prime}}\right|^{2} \\
& =\left|\lambda Y Y^{\prime}+M_{P Q}^{2} \kappa_{1} \cdot q\right|^{2}+\left|\lambda X Y^{\prime}\right|^{2}+|\lambda X Y|^{2}
\end{aligned}
$$

\subsection{Fayet-Polonyi Model}

We now determine the scale of supersymmetry breaking in our realization of the Fayet-Polonyi model on a PQ seven-brane. In particular, we show that this theory can accommodate the vev of $X$ required for the gauge mediation scenario explored in this paper.

The effective potential for the $X$ field is given by the sum of $V_{\text {Fayet }}$ and $V_{\text {Polonyi }}$ :

$$
\begin{aligned}
V_{P Q} & =V_{\text {Fayet }}+V_{\text {Polonyi }} \\
& =2 \pi \alpha_{P Q} \cdot\left(|X|^{2}+\sum_{\Psi} e_{\Psi}|\Psi|^{2}-K^{\prime}\left(\mathcal{C}+\mathcal{C}^{\dagger}\right)+\xi_{\text {flux }}\right)^{2} \\
& +\left|\lambda Y Y^{\prime}+M_{P Q}^{2} \kappa_{1} \cdot q\right|^{2}+\left|\lambda X Y^{\prime}\right|^{2}+|\lambda X Y|^{2}
\end{aligned}
$$

By inspection, $V_{P Q}$ admits critical points where all fields other than $X$ and $\mathcal{C}$ vanish. Because a non-zero vev for such fields would break at least part of the GUT group, we shall only consider vacua where $X$ and $\mathcal{C}$ have non-trivial vevs.

Returning to equation (8.21), $\partial W / \partial X \neq 0$ so that supersymmetry is broken. 
Working to leading order in $q$, the vev $\langle X\rangle=x+\theta^{2} F$ satisfies:

$$
\begin{aligned}
|x|^{2} & =\xi_{P Q} \\
\frac{\bar{F}}{M_{P Q}^{2}} & =\kappa_{1} \cdot q .
\end{aligned}
$$

To estimate the value of $x$, recall that in terms of the background field strength on $S_{P Q}$, we have:

$$
|x|^{2}=\xi_{P Q}=\xi_{\text {flux }}+\xi_{*} .
$$

Note that in the absence of any matter curves, the Hermitian Yang-Mills equations on $S_{P Q}$ would imply:

$$
\omega_{P Q} \wedge F_{P Q}=0
$$

The presence of the matter curves allows $\omega_{P Q} \wedge F_{P Q}$ to deviate away from zero. In vacua where $x$ is non-zero, we therefore expect that the background $U(1)_{P Q}$ gauge field configuration will effectively adjust itself so that $\xi_{P Q}$ is as close to zero as possible. At generic points of $\Sigma_{X}$, the field strength $F_{P Q}$ will scale as $M_{X}^{2}$. Assuming that the Planck length is the minimal distance over which $F_{P Q}$ can vary by the amount $M_{X}^{2}$, it follows that the minimal non-zero value of $\xi_{P Q}$ which can be attained is:

$$
\xi_{\min }=\Delta_{\min }^{2} \cdot M_{X}^{2}
$$

where:

$$
\Delta_{\min }=\frac{M_{X}}{M_{p l}} \sim 10^{-3.5}
$$

is the effective lattice spacing for Peccei-Quinn flux configurations. Throughout this paper, we have assumed that $M_{X} \sim 10^{15.5} \mathrm{GeV}$ and $M_{p l} \sim 10^{19}$ which implies:

$$
|x| \sim \Delta_{\min } \cdot M_{X} \sim 10^{12} \mathrm{GeV}
$$

Remarkably, this simple estimate is in accord with the requirements of both gauge mediation and axion physics! Recall that the solution of $\mu / B \mu$ problem using the Giudice-Masiero operator required that $|x| / M_{X} \ll 1$. We now see that this is related to

$$
\frac{|x|}{M_{X}} \sim \Delta_{\min } \sim \frac{M_{G U T}}{M_{p l}} \ll 1 .
$$


Indeed, this mild hierarchy between the GUT scale and the Planck scale played a key role in some of the estimates of physical quantities (for example the neutrino masses) in [2].

Computing the scale of supersymmetry breaking $\sqrt{F}$ is somewhat more delicate because the instanton action depends exponentially on the volume of the Kähler surface $S_{P Q}$. For this reason, we shall instead determine the mass scale $M_{P Q}$ required in order to achieve the value $F \sim 10^{17} \mathrm{GeV}^{2}$. To estimate the size of the instanton factor, we introduce a characteristic volume factor $V$ so that $q=\exp \left(-M_{*}^{4} \cdot V\right)$. Similarly, we shall introduce a characteristic mass scale $M \sim V^{-1 / 4}$. Returning to equation (8.29), the magnitude of $F$ is then:

$$
\frac{|F|}{M_{P Q}^{2}}=\exp \left(-M_{*}^{4} \cdot V\right)=\exp \left(-\frac{2 \pi}{\alpha_{G U T}} \frac{M_{G U T}^{4}}{M^{4}}\right)
$$

where in the first equality we have set $\kappa_{1}=1$, and in the second equality we have used the relation between the volumes of surfaces and gauge couplings for seven-branes discussed in $[1,2]$. Assuming that $M_{P Q} \sim M$, solving for $M$ thus yields:

$$
\frac{M}{M_{G U T}}=\left(\frac{\alpha_{G U T}}{4 \pi} \times W\left(\frac{4 \pi}{\alpha_{G U T}} \cdot \frac{M_{G U T}^{4}}{F^{2}}\right)\right)^{-1 / 4}
$$

where $W$ is the Lambert $W$-function. Setting $\alpha_{G U T} \sim 1 / 25, F \sim 10^{17} \mathrm{GeV}^{2}$ and $M_{G U T} \sim 3 \times 10^{16} \mathrm{GeV}$, we find:

$$
\frac{M}{M_{G U T}} \sim 1.4
$$

In other words, with only a mild tuning of parameters in the geometry, the anomalous $U(1)_{P Q}$ gauge theory achieves the scale of supersymmetry breaking required to remain in accord with weak scale physics. Returning to equation (8.36) and using the value $|F| \sim 10^{17} \mathrm{GeV}^{2}$ the instanton action is therefore given by:

$$
|q| \sim \frac{|F|}{M_{P Q}^{2}} \sim 5 \times 10^{-17}
$$

To conclude this subsection, we note that more generally, the value of $\kappa_{1}$ could deviate from an order one number, so there is a certain degree of tunability in such 
instanton contributions. Indeed, in general F-theory compactifications, the value of $\tau_{\text {IIB }}$ can vary along the three-fold base $B_{3}$.

\subsection{Stabilizing the Radial Mode}

In the previous sections we have seen that the parameter space of F-theory GUTs is in principle compatible with a non-zero vev for $X$ and $q$ of the form:

$$
\begin{aligned}
|X| & \sim 10^{12} \mathrm{GeV} \\
\log |q| & \sim \log \frac{F}{M_{P Q}^{2}} \simeq-38 .
\end{aligned}
$$

On the other hand, the contribution from just the Polonyi terms and D-term potential does not by itself stabilize the PQ invariant product:

$$
\widehat{X} \equiv q X
$$

Indeed, at low energy scales, there is no D-term potential, as the effects of the $U(1)_{P Q}$ gauge boson have been integrated out, leaving behind only an accidental global $U(1)$ at low energies. The phase of $\widehat{X}$ corresponds to the QCD axion, which develops a potential through QCD instanton effects, and possibly instanton contributions from the PQ seven-brane. The norm corresponds to the other bosonic component of the chiral multiplet which is the saxion.

In this section we discuss how the norm of $\widehat{X}$ can develop a vev compatible with the supersymmetry breaking conditions specified earlier. Non-trivial contributions to the Kähler potential in theories with an anomalous $U(1)$ symmetry can often serve to stabilize the vevs of modes such as $\widetilde{X}$ which are not fixed by the Polonyi term alone [44]. Our aim here will be to clarify the form of fine-tuning necessary to achieve the required energy scales. To this end, we first phrase in general terms the requisite conditions on the form of the Kähler potential, and then show in a well-motivated example that these conditions can be met.

To analyze the dynamics of $\widehat{X}$, it is convenient to consider the unbroken PQ sevenbrane gauge theory, which will contain the dynamical fields $X$ and $q$. In principle, the FI parameter $\xi_{\text {flux }}$ should also be included as a dynamical field, but this mode involves the dynamics of the full ten-dimensional theory, and so for our purposes can 
effectively be treated as a frozen parameter.

We begin by discussing the general features of the system defined by $q, X$, and the PQ gauge boson. The holomorphic coupling $\tau$ of the PQ seven-brane theory is related to $q$ through:

$$
q=\exp (2 \pi i \tau) \equiv \exp (-S)
$$

For simplicity, we work in units where the PQ charge of the $X$ field is +1 , so that under a gauge transformation which shifts the PQ vector multiplet as:

$$
V_{P Q} \rightarrow V_{P Q}-\Lambda-\Lambda^{\dagger}
$$

the $X$ field transforms as:

$$
X \rightarrow \exp (\Lambda) \cdot X
$$

Gauge invariance of the product $q X$ then requires $q$ to transform as:

$$
q \rightarrow \exp (-\Lambda) \cdot q
$$

or:

$$
S \rightarrow S+\Lambda
$$

The theory with the PQ gauge boson can then be parameterized in terms of the contribution from the Kähler potential and superpotential for $S$ and $X$ :

$$
L \supset \int d^{4} \theta K\left(X^{\dagger} e^{V_{P Q}} X ; S+S^{\dagger}+V_{P Q}\right)+\xi_{f l u x} V_{P Q}+\int d^{2} \theta M_{P Q}^{2} e^{-S} X+h . c .,
$$

where the general form we have taken is automatically invariant under gauge transformations.

To leading order, we approximate $K$ as the sum of two contributions, $K_{X}$ and $K_{S}$ such that:

$$
K\left(X^{\dagger} e^{V_{P Q}} X ; S+S^{\dagger}+V_{P Q}\right)=K_{X}\left(X^{\dagger} e^{V_{P Q}} X\right)+K_{S}\left(S+S^{\dagger}+V_{P Q}\right) .
$$

Expanding to quadratic order in $V_{P Q}$, the mass of the $\mathrm{PQ}$ gauge boson is given as:

$$
M_{U(1)_{P Q}}^{2}=X^{\dagger} X \cdot K_{X}^{\prime}+\left(X^{\dagger} X\right)^{2} \cdot K_{X}^{\prime \prime}+K_{S}^{\prime \prime},
$$


where the primes on $K_{X}$ and $K_{S}$ respectively denote derivatives of the Kähler potentials. To leading order, the mass of the PQ gauge boson is controlled by $K_{S}^{\prime \prime}$, which can in principle be tuned from a scale close to the GUT scale, to somewhat lower values.

Since the mass of the PQ gauge boson is far heavier than the scale of supersymmetry breaking, we can view the D-term potential as imposing a constraint on the norms $|X|$ and $|q|$. Explicitly, the D-term constraint requires:

$$
X^{\dagger} X \cdot K_{X}^{\prime}+K_{S}^{\prime}+\xi_{\text {flux }}=0
$$

Assuming $K_{X}^{\prime}>0$ in the regime of interest (which will be the case when $K_{X}$ is to leading order given by the canonical Kähler potential), note that $\xi_{\text {flux }}>0$ favors field configurations such that $X=0$ and $K_{S}^{\prime} \simeq-\xi_{\text {flux }}$. Indeed, this is just a rephrasing of the Bousso-Polchinski flux scanning argument that $K_{S}^{\prime}$ and $\xi_{\text {flux }}$ should both be large and nearly cancel in order for $|X|$ to remain below the GUT scale.

Next consider the F-term contribution to the potential:

$$
V_{F-t e r m}=\left|M_{P Q}^{2} e^{-S}\right|^{2}\left(g^{X \bar{X}}+g^{S \bar{S}}|X|^{2}\right)
$$

The overall multiplicative factor by $\exp \left(-S-S^{\dagger}\right)$ indicates the tendency for this mode to approach $S \rightarrow \infty$, which is a possible "runaway direction". This is counteracted, however, by the D-term constraint of equation (8.51). We are interested in the form of $V_{F-t e r m}$ such that $|X|$ is small in $M_{*}$ units, and $S$ is large. This can be achieved provided the contributions from $g^{X \bar{X}}$ and $g^{S \bar{S}}|X|^{2}$ are roughly comparable in size. Assuming a roughly canonical form for the Kähler potential of $X$, this means that the Kähler metric $g_{S \bar{S}}$ must be small in $M_{*}$ units so that $|X|^{2} / g_{S \bar{S}}$ is comparable to $g^{X \bar{X}}$. Note, however, that $g_{S \bar{S}}$ also enters into the mass of the PQ gauge boson, and so cannot be too small.

The mass of the mode stabilized by $V_{F-t e r m}$ is naturally close to the weak scale. Indeed, the characteristic mass scale for fluctuations of $\widehat{X}$ are given as:

$$
m_{\widehat{X}}^{2} \sim\left|M_{P Q}^{2} e^{-S}\right|^{2} g^{S \bar{S}}=\left|\frac{F}{M_{U(1)_{P Q}}}\right|^{2} \propto \Delta_{P Q}^{2} .
$$

Thus, we can naturally expect the mass of the radial mode to be determined by the 
scale of the PQ deformation. The precise numerical coefficient depends on details of precisely how the vevs of $X$ and $q$ are fixed, but the basic point remains that the mass of the radial component is near the weak scale, while the phase, corresponding to the axion will have a much lower mass induced by QCD instanton effects.

Having spelled out the general form of the required conditions, we now show that well-motivated Kähler potentials $K_{X}$ and $K_{S}$ exhibit the behavior required to achieve the desired minimum. This will also help to clarify at least in this particular example the precise type of fine-tuning at work in the Bousso-Polchinski flux scanning argument. For simplicity, we consider the case where the Kähler potential of $S$ is given as:

$$
K_{S}=-M_{*}^{2} \log \left(S+S^{\dagger}\right)
$$

This type of logarithmic behavior for $K_{S}$ is typically present for complex surfaces which contract to a point at infinite distance in moduli space. A different form based on a power law dependence is instead more natural if this contraction occurs at finite distance in moduli space. In addition, we shall consider a roughly canonical form for $K_{X}$ which allows for higher order corrections:

$$
K_{X}=A X^{\dagger} X+B \frac{\left(X^{\dagger} X\right)^{2}}{M_{X}^{2}}+O\left(\frac{\left(X^{\dagger} X\right)^{3}}{M_{X}^{4}}\right)
$$

where the suppression scale $M_{X}$ is associated with integrating out massive modes of size $M_{X}$ localized on the $X$ curve. The specific value of the coefficient $B$ will in general receive corrections of both signs. In the context of similar couplings between $X$ and MSSM fields, the dominant contribution of this type is from heavy PQ gauge boson exchange. In the context of purely $X$ contributions, however, the somewhat lower mass scale $M_{X}$ allows for a more general possibility.

We now proceed to analyze the form of the effective potential in this case. First consider the special case where we drop all higher order corrections to $K_{X}$ in equation (8.55). The form of the D-term constraint is now:

$$
A|X|^{2}-\frac{M_{*}^{2}}{S+S^{\dagger}}+\xi_{\text {flux }}=0
$$


while the F-term potential is given as:

$$
V_{F-\text { term }}=M_{P Q}^{4} \exp \left(-S-S^{\dagger}\right)\left(\frac{1}{A}+\left(S+S^{\dagger}\right)^{2} \frac{|X|^{2}}{M_{*}^{2}}+O\left(|X|^{4}\right)\right)
$$

where $V_{F-t e r m}$ is to be viewed as a potential for the mode unfixed by the D-term constraint. Parameterizing the form of $V_{F-t e r m}$ in terms of $|X|$, we obtain:

$$
\begin{aligned}
V_{F-\text { term }} & =M_{P Q}^{4} \exp \left(-\frac{M_{*}^{2}}{A|X|^{2}+\xi_{\text {flux }}}\right) \cdot\left(\frac{1}{A}-\frac{M_{*}^{2}}{A|X|^{2}+\xi_{\text {flux }}} \frac{|X|^{2}}{M_{*}^{2}}+\ldots\right) \\
& \simeq M_{P Q}^{4} \exp \left(-\frac{M_{*}^{2}}{\xi_{\text {flux }}}\right) \cdot\left(\frac{1}{A}+\frac{\left(M_{*}^{2}-\xi_{\text {flux }}\right)}{\xi_{\text {flux }}^{2}}|X|^{2}+\ldots\right)
\end{aligned}
$$

where we have dropped all terms of order $|X|^{4}$ to obtain a consistent approximation which neglects the terms proportional to $B$ in $K_{X}$. The form of $V_{F-t e r m}$ is quadratic, leading to a stable minimum at $|X|=0$. To leading order, this is encouraging, because we are interested in potentials with $X$ stabilized at values below the GUT scale.

Including higher order corrections can shift the minimum of $V_{F-t e r m}$ so that a small non-zero vev for $X$ is indeed realized. Including the first correction proportional to $B$ and expanding to order $|X|^{4}$ now yields:

$$
V_{F-\text { term }} \simeq M_{P Q}^{4} \exp \left(-\frac{M_{*}^{2}}{\xi_{\text {flux }}}\right) \cdot\left(\alpha+\beta|X|^{2}+\gamma|X|^{4}+\ldots\right),
$$

where the coefficients $\alpha, \beta$ and $\gamma$ are:

$$
\begin{aligned}
& \alpha=\frac{1}{A} \\
& \beta=\frac{\left(M_{*}^{2}-\xi_{\text {flux }}\right)}{\xi_{\text {flux }}^{2}}-\frac{4 B}{A^{2}} \frac{1}{M_{X}^{2}} \\
& \gamma=\frac{A}{\xi_{\text {flux }}^{4}}\left(\left(M_{*}^{2}-\xi_{\text {flux }}\right)^{2}-\frac{M_{*}^{4}}{2}\right)-\frac{2 B}{A^{2}} \frac{M_{*}^{2}}{M_{X}^{2} \cdot \xi_{\text {flux }}^{2}}+\frac{16 B^{2}}{A^{3}} \frac{1}{M_{X}^{4}} .
\end{aligned}
$$

An important feature of the above relations is that depending on the size of $B$, the coefficient $\beta$ can be either positive or negative. In particular, this illustrates that 


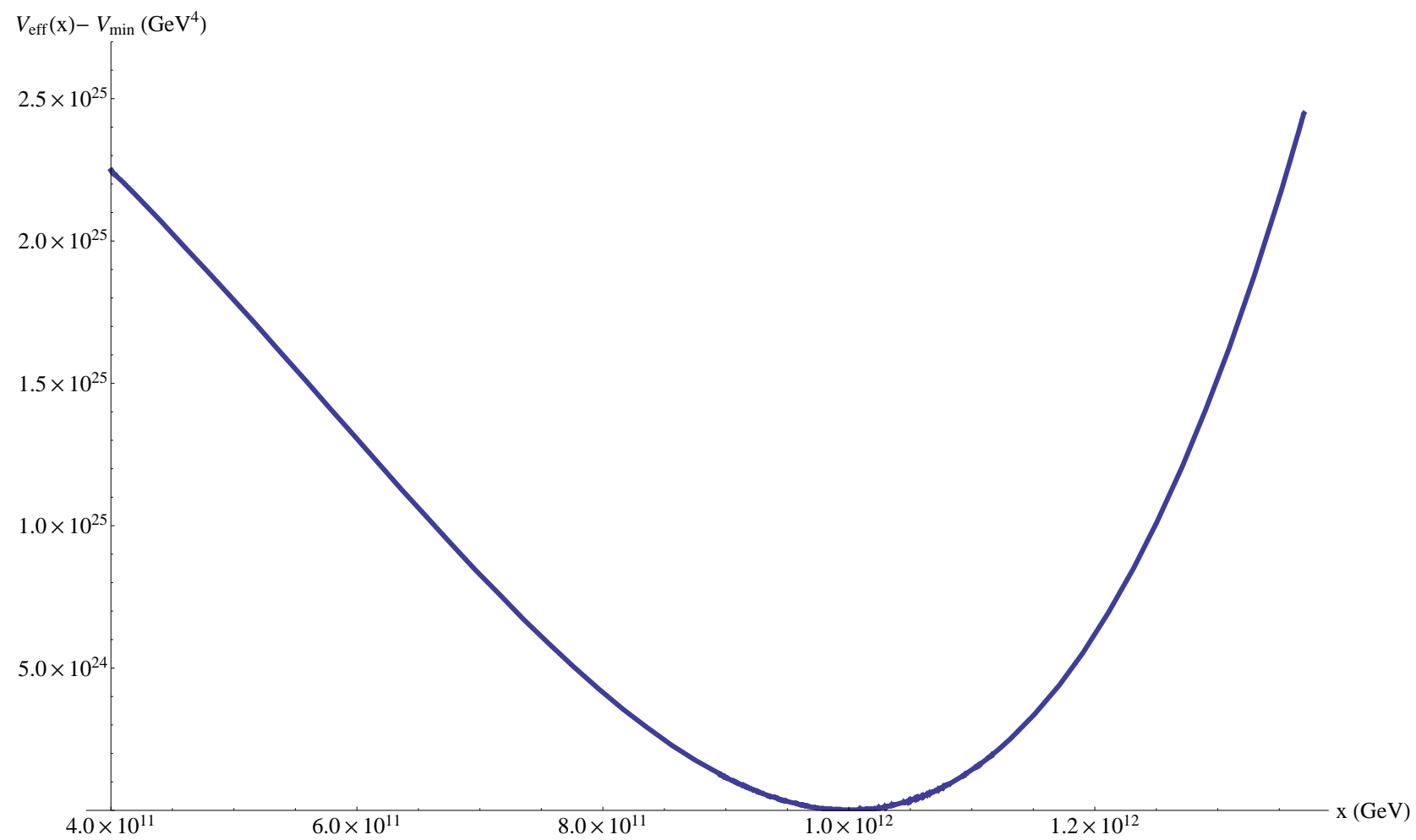

Figure 3: Plot of the effective potential for the saxion. With notation as in section 8.4, the specific choice of parameters used in this plot are $M_{*}=10^{17} \mathrm{GeV}, M_{X}=10^{15.5}$ $\mathrm{GeV}, A=1, B=1.425$. By construction, the value of $B$ has been chosen so that the minimum is located at $x_{*}=10^{12} \mathrm{GeV}$. The shallow variation of the potential as a function of energy scale illustrates that the mass of this radial mode is much smaller than $10^{12} \mathrm{GeV}$, and is instead closer to the weak scale. 
provided $B$ is large enough, we can expect a shift in the minimum of $V_{F-t e r m}$ to a small, nearby value $x_{*}$ given by:

$$
\left|x_{*}\right|^{2}=\frac{A^{2} M_{X}^{2} \xi_{\text {flux }}+4 B \xi_{\text {flux }}^{2}-A^{2} M_{X}^{2} M_{*}^{2}}{2 A^{3} M_{X}^{2}+32 \frac{B^{2} \xi_{\text {flux }}^{2}}{A M_{X}^{2}}-4 A B M_{*}^{2}-\frac{4 A^{3} M_{X}^{2} M_{*}^{2}}{\xi_{\text {flux }}}+\frac{A^{3} M_{X}^{2} M_{*}^{4}}{\xi_{\text {flux }}^{2}}} .
$$

To obtain representative values for the size of the various coefficients, note that the mass scale $M_{X} \sim 10^{15.5} \mathrm{GeV}$ is typically somewhat smaller than $M_{*} \sim 10^{17} \mathrm{GeV}$. As a representative example, we shall take:

$$
\frac{M_{X}}{M_{*}} \sim 10^{-1.5}
$$

Numerically, the value of $\xi_{\text {flux }}$ is fixed by the condition that the value of $S$ generates an appropriate instanton action. Taking the rough value specified by equation (8.41), this imposes the condition set by the D-term constraint:

$$
\xi_{\text {flux }} \sim \frac{M_{*}^{2}}{76} .
$$

Achieving the required value of $\left|x_{*}\right| \sim 10^{12} \mathrm{GeV}$ then leads to a specific relation between $A$ and $B$. For example, with $M_{X} \sim 10^{15.5} \mathrm{GeV}$ and $M_{*} \sim 10^{17} \mathrm{GeV}$ and $A=1, B \sim 1.4$. The coefficient $A$ is typically a number somewhat larger than one, since the kinetic term for $X$ scales as $A \sim M_{*}^{2} \operatorname{Vol}\left(\Sigma_{X}\right)$, which is naturally on the order of $100-1000$. When $A \sim 100$, we instead find $B \sim 1.4 \times 10^{4}$. Although this constitutes a fine-tuning, note that the ratio $B / A^{2}$ remains an order one number.

\section{Region of MSSM Parameter Space}

In previous sections, we have shown that low energy considerations constrain the UV boundary conditions for the supersymmetry breaking sector. In particular, we have found that for a broad class of F-theory compactifications, the vev $\langle X\rangle=x+\theta^{2} F$ satisfies crude constraints from the weak scale when $x \sim 10^{12}$ and $F \sim 10^{17} \mathrm{GeV}^{2}$. Based on such considerations, we can deduce that if supersymmetry breaking is communicated to the MSSM via gauge mediation, then the LSP will be the gravitino. In a purely top down approach, this level of analysis is essentially all that can be 
obtained with any degree of certainty. Indeed, deriving detailed features of the low energy spectrum would require specifying all relevant interaction terms in both the F- and D-terms, which at a purely practical level may not even be feasible.

Even if such a computation were in principle possible, there is no guarantee that the resulting low energy physics would match to observation. Adhering to the bottom up approach advocated in the Introduction, in this section we show that simply achieving the correct low energy behavior in the Standard Model strongly constrains both the sparticle spectrum, as well as properties of the Higgs potential. Because we do not know their precise values, our strategy will be to scan over a range of UV boundary conditions in gauge mediation models close to the crude values specified in previous sections. In addition, we also include the effects of the one parameter deformation away from gauge mediation determined by heavy $U(1)_{P Q}$ gauge boson exchange. We find that over this region, some parameters of the low energy theory do not vary much, while others are more sensitive to high energy inputs. To simplify the presentation, we shall confine our discussion to the case of a single vector-like pair of messenger fields in the $5 \oplus \overline{5}$ of $S U(5)$. The primary change in increasing the number of messengers is that as the number of messenger fields increase, the NLSP can transition from the bino-like neutralino to the stau.

To determine the low energy behavior of the theory, we have developed a small modification of the program SOFTSUSY [29]. Once an appropriate subset of UV boundary conditions has been specified, SOFTSUSY performs a renormalization group flow of the parameters of the theory down to the weak scale, adjusting the values of parameters such as the $\mu$ and $B \mu$ terms as well as $\tan \beta$ near the weak scale to remain consistent with electroweak symmetry breaking. More precisely, this is accomplished by requiring that the masses squared for the Higgs fields develop a suitable tachyonic value. The program then evolves these parameters back up to the messenger scale, and iterates this procedure until the results of these adjustments converge. In a certain sense, this represents a microcosm for the entire bottom up approach to string phenomenology. Indeed, the reason why the bottom up approach is in principle quite predictive is that while crude considerations from UV or IR physics may only serve to partially fix some details of the theory, iterating back and forth between high and low scale physics can effectively constrain both sectors to a high level of precision. Indeed, using this fact, we will be able to extract remarkably detailed information which directly correlates the high energy behavior of the theory 
with low energy physics.

The remainder of this section is organized as follows. In subsection 9.1, we specify in greater detail the UV boundary conditions which we shall scan over, including contributions from heavy $U(1)_{P Q}$ gauge boson exchange. Next, we turn to the low energy behavior of the theory in subsection 9.2. In particular, we show that achieving consistent electroweak symmetry breaking effectively allows us to reliably extract the value of $\mu$ and $\tan \beta$ near the scale of electroweak symmetry breaking. Having determined the precise values of all UV boundary conditions, we next present the low energy spectra for this class of models. In nearly all gauge mediation models, the LSP is the gravitino, and our situation is no different 9 Similarly, with a single vector-like pair of messenger fields in the $5 \oplus \overline{5}$ of $S U(5)$, for the most part, the NLSP is given by a bino-like neutralino, although the one parameter deformation from gauge mediation can also allow the stau to become the NLSP. Finally, we conclude this section with a brief discussion of the mini-hierarchy problem. As in most models, we find that a fine-tuning on the order of one part in a hundred is required to remain in accord with present experimental bounds, and including our deformation away from gauge mediation appears to only improve this situation slightly.

\subsection{UV Boundary Conditions}

The low energy content of any mediation model is completely fixed once all soft breaking parameters have been specified at the messenger scale. For gauge mediation models, this amounts to specifying the number of messenger fields, the messenger mass scale(s) $M_{m e s s}$, the gaugino mass unification scale $\Lambda=F / x$, and the values of $\mu, B \mu$ and $\tan \beta$. The $\mathrm{PQ}$ deformation can also contribute to the soft terms, and in certain cases will lead to important deviations from the usual predictions of gauge mediation. As explained in subsection 4.2, the size of this contribution is a priori not fixed by purely local considerations, and it is therefore appropriate to scan over the allowed range of soft mass terms to the Higgs fields $H_{i}$ and all other chiral

\footnotetext{
${ }^{9}$ For an interesting recent example of a gauge mediation scenario where the gravitino is not the LSP, see [69].
} 
superfields $\Phi$ of the MSSM induced by the PQ deformation:

$$
\begin{aligned}
\delta_{P Q} m_{H_{i}}^{2}\left(M_{\text {mess }}\right) & =2 \Delta_{P Q}^{2} \\
\delta_{P Q} m_{\Phi}^{2}\left(M_{m e s s}\right) & =-\Delta_{P Q}^{2}
\end{aligned}
$$

where $\Delta_{P Q}$ denotes the mass scale associated with the PQ deformation, and the relative sign and magnitude is completely fixed by the relative $U(1)_{P Q}$ charges of these fields. The UV boundary conditions for our model are given by:

$$
\begin{aligned}
M_{\text {mess }} & \sim 10^{11.5}-10^{12.5} \mathrm{GeV} \\
\Lambda & \sim 10^{5}-10^{6} \mathrm{GeV} \\
B \mu\left(M_{\text {mess }}\right) & =0 \\
\mu & \sim \pm 10^{2}-10^{3} \mathrm{GeV} \\
\Delta_{P Q} & \sim 0-10^{3} \mathrm{GeV} .
\end{aligned}
$$

In addition, the A-terms vanish at the messenger scale. In the above, our conventions for the sign of the $\mu$ term are the same as in [70]. Here, we have specified all requisite inputs for the theory at the messenger scale, and have also included a potential range of values over which we shall perform our scan of UV boundary conditions. Because these boundary conditions completely fix the soft breaking parameters of the MSSM, some points in this parameter space may not be consistent with current experimental bounds on the mass of either the Higgs or the sparticles of the MSSM.

\subsection{Constraining the MSSM}

To extract detailed properties of the low energy spectrum which are consistent with electroweak symmetry breaking, we have used the SOFTSUSY package [29], and have also included a small modification which incorporates the specific form of the PQ deformation in the present class of models. The inputs at the messenger scale are given in terms of $M_{\text {mess }}, \Lambda, \tan \beta\left(M_{\text {mess }}\right), \Delta_{P Q}$ and the sign of the $\mu$ term. Once these parameters are fixed, all other UV boundary conditions are automatically adjusted by the algorithm to remain in accord with electroweak symmetry breaking. A cursory inspection of the output reveals that the spectrum is relatively insensitive to the value of $M_{\text {mess }}$ over the range of values $10^{11.5}-10^{12.5}$. With little loss of 
precision, it is therefore sufficient to fix $M_{\text {mess }}=10^{12} \mathrm{GeV}$. Similarly, for the most part the sign of $\mu$ does not appear to significantly alter the results of our analysis, and we shall therefore restrict to the case $\mu>0$.

Within the three-dimensional subspace parameterized by $\Lambda, \tan \beta\left(M_{\text {mess }}\right)$ and $\Delta_{P Q}$, only a two-dimensional subspace will in general preserve the required boundary condition $B \mu\left(M_{\text {mess }}\right)=0$. To simplify our analysis, we fixed a particular value of either $\Lambda$ or $\Delta_{P Q}$ and then scanned over the remaining two parameters to determine the value of $\tan \beta\left(M_{\text {mess }}\right)$. While the value of $\tan \beta\left(M_{\text {mess }}\right)$ does depend on $\Lambda$, it is relatively insensitive to the value of $\Delta_{P Q}$. To determine the effect of the PQ deformation, it therefore suffices to fix all other UV boundary conditions, and simply vary the size of $\Delta_{P Q}$.

The particular choice of values for $\Lambda \sim 10^{5}-10^{6} \mathrm{GeV}$ which we scan over is in large part a consequence of current experimental bounds on the mass of the lightest neutral Higgs $h^{0}(114.5 \mathrm{GeV})$. In addition, the size of the deformation $\Delta_{P Q} \sim 0-1000 \mathrm{GeV}$ is also bounded above by the requirement that none of the squarks or sleptons should develop a tachyonic mode near the weak scale. We note that as $\Lambda$ increases, the soft scalar masses will also increase, so that larger PQ deformations become viable.

We present the results of various one parameter scans over $\Lambda$ and $\Delta_{P Q}$. One scan is performed at vanishing $\mathrm{PQ}$ deformation so that $\Delta_{P Q}=0$, with $\Lambda$ ranging from $10^{5}$ to $10^{6} \mathrm{GeV}$. At the low end of this scan, the resulting Higgs mass in fact lies below the experimental bound set by LEP that $m_{h^{0}}>114.4 \mathrm{GeV}$ at the 95 percent confidence level [71]. The mass of the Higgs monotonically increases with $\Lambda$, and saturates the value $114.5 \mathrm{GeV}$ at $\Lambda=10^{5.08} \mathrm{GeV}$. In figure 4 we display $\tan \beta\left(M_{S}\right)$ as a function of $\Lambda$. Here, $M_{S}$ denotes the scale at which electroweak symmetry breaking boundary conditions are imposed. By inspection, $\tan \beta$ increases logarithmically with $\Lambda$ from $\sim 25$ near $\Lambda=10^{5.08} \mathrm{GeV}$ to $\sim 38$ near $\Lambda=10^{6} \mathrm{GeV}$ so that $\tan \beta\left(M_{S}\right) \sim 31.5 \pm 6.5$. In the present single messenger model, the NLSP is given by a bino-like neutralino, while the stau is the next lightest MSSM sparticle. See figure 5 for a plot of the bino mass, stau mass and Higgs mass as a function of $\Lambda$. In this same plot, we also present the value of the $\mu$ term as a function of $\Lambda$. At its lowest value, $\mu=10^{2.8} \sim$ $630 \mathrm{GeV}$ for $\Lambda=10^{5.08} \mathrm{GeV}$, and the value of $\mu$ increases to $10^{3.6} \sim 4000 \mathrm{GeV}$ for $\Lambda=10^{6} \mathrm{GeV}$.

We have also scanned over a range of values for the PQ deformation with $\Lambda$ kept fixed. To leading order this deformation does not alter the masses of any 


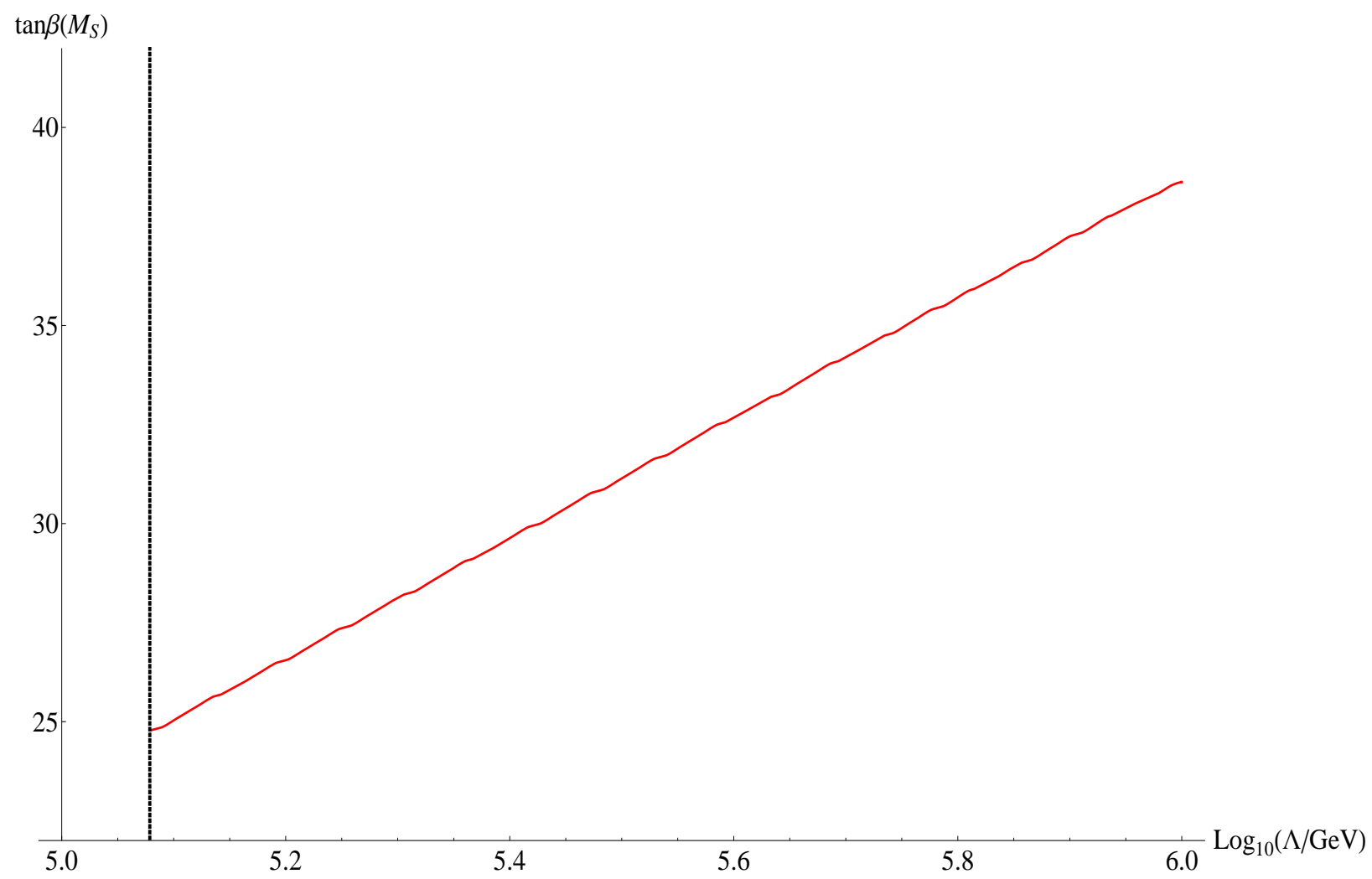

Figure 4: Plot of $\tan \beta$ at the scale $M_{S}$, the scale at which electroweak symmetry breaking boundary conditions are imposed, as a function of $\log _{10}(\Lambda / \mathrm{GeV})$. By inspection, $\tan \beta$ grows logarithmically with the gaugino mass unification scale. The vertical line at the left of the plot $\left(\Lambda=10^{5.08} \mathrm{GeV}\right)$ indicates the experimentally excluded region based on current bounds on the mass of the Higgs. 


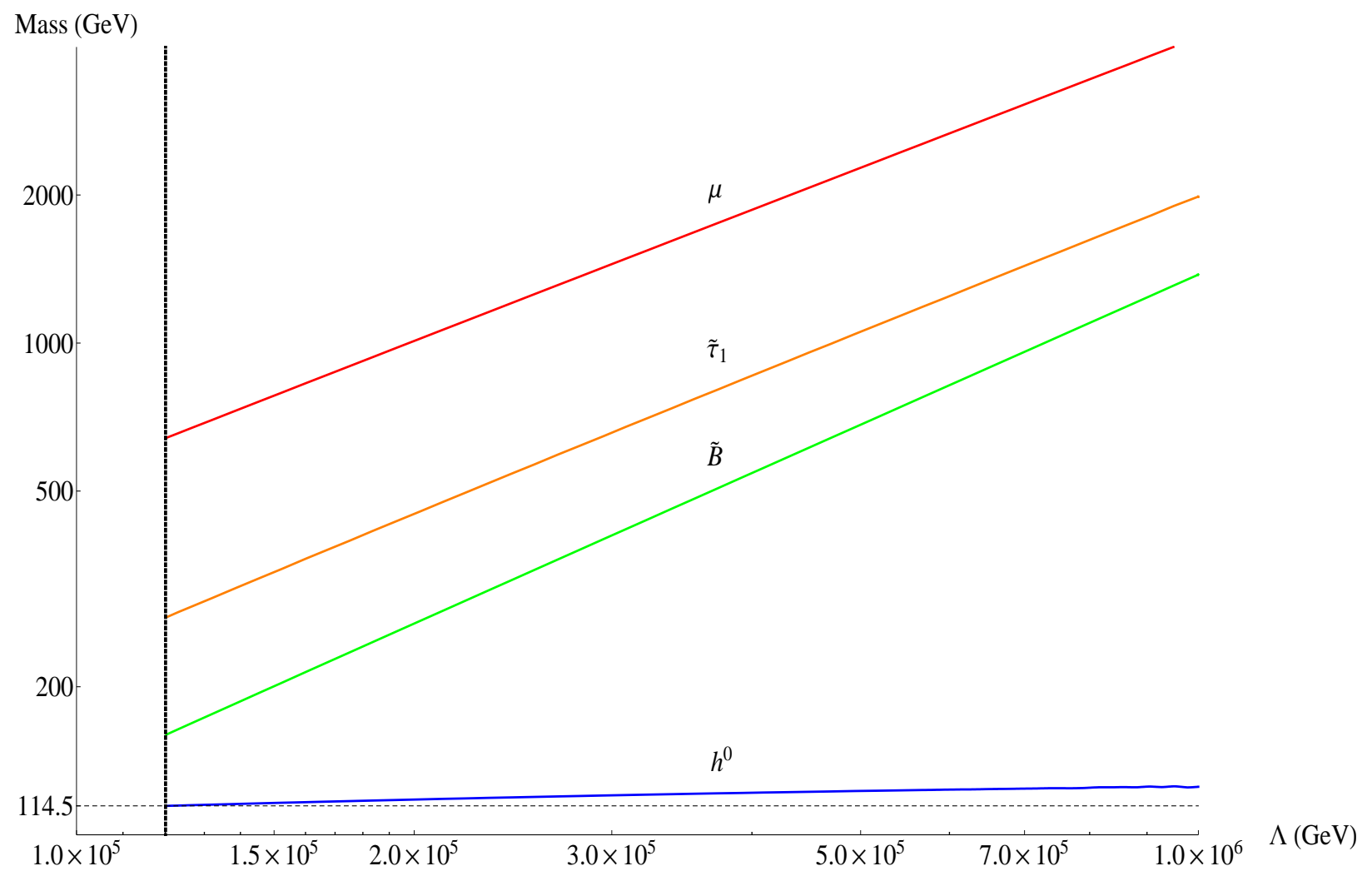

Figure 5: Plot of the $\mu$ term, stau mass, bino mass and Higgs mass as a function of the gaugino mass unification scale $\Lambda$ in a single messenger model with vanishing PQ deformation. The vertical line at the left $\left(\Lambda=10^{5.08} \mathrm{GeV}\right)$ indicates the experimentally excluded region based on bounds on the mass of the Higgs. 
gauginos of the theory but does lower the masses of all sleptons and squarks whilst increasing the soft masses squared of the Higgs fields. Recall that in order for the Higgs potential to contain a tachyonic mode, the mass squared of the Higgs fields undergoes a renormalization group flow from positive values to negative values. As the PQ deformation increases, the magnitude of this tachyonic mode consequently also decreases.

As a representative example, we scanned over the PQ deformation for a fixed value of $\Lambda=5 \times 10^{5} \mathrm{GeV}$. This scan begins at $\Delta_{P Q}=0$ and proceeds up to $\Delta_{P Q}=10^{2.9} \mathrm{GeV}$. For larger values of $\Delta_{P Q}$, the corresponding effective potential for the sfermions contains a tachyonic mode. Over this entire range, the resulting value of $\tan \beta\left(M_{S}\right)$ is $34 \pm 1$, and the mass of the lightest Higgs $h^{0}$ is approximately $123 \pm 3 \mathrm{GeV}$. See figure 6 for a plot of the parameter $\mu$ as a function of $\Delta_{P Q}$. As the value of $\Delta_{P Q}$ increases, $\mu$ decreases slightly. A more dramatic consequence of the $\mathrm{PQ}$ deformation is shown in figure 7 which shows that the mass of the stau becomes nearly equal to the bino at large values of the PQ deformation.

For smaller values of $\Lambda$, the stau can become the NLSP at large values of the PQ deformation. Performing a scan over the PQ deformation at the value $\Lambda=1.3 \times 10^{5}$ $\mathrm{GeV}$, we find that $\Delta_{P Q}$ can range from zero up to $290 \mathrm{GeV}$, beyond which point a tachyon develops in the scalar potential. Over this entire range, the resulting value of $\tan \beta\left(M_{S}\right)$ is $26 \pm 1$, and the mass of the lightest Higgs $h^{0}$ is $115 \pm 1 \mathrm{GeV}$. The profile of the parameter $\mu$ is similar in shape to that given in figure [6, although the ranges of the scales are different. At large values of the $\mathrm{PQ}$ deformation, the value of $\mu$ is $550 \mathrm{GeV}$. In figure 8 we plot the four lightest sparticles and find that at a value of $\Delta_{P Q}=243 \mathrm{GeV}$, the mass of the stau and bino-like neutralino are identical. For a narrow range of values, the stau becomes lighter. In addition, the mass of the right-handed selectron and smuon become comparable in mass to the bino-like neutralino at large PQ deformation. This example explicitly shows that that for a fixed value of $\Lambda$, increasing the size of $\Delta_{P Q}$ can allow the stau to become the NLSP.

It is also of interest to compare the entire sparticle spectrum in the limit of vanishing $\mathrm{PQ}$ deformation, as well as in the presence of maximal PQ deformation. Because the resulting value of $\tan \beta\left(M_{S}\right)$ is relatively insensitive to $\mathrm{PQ}$ deformations, in figure 9 we directly compare the sparticle spectrum at $\Lambda=1.3 \times 10^{5} \mathrm{GeV}$ for $\Delta_{P Q}=$ 0 with the spectrum obtained with a PQ deformation of $\Delta_{P Q}=290 \mathrm{GeV}$. Beyond this value, a tachyonic mode is generically present in the sfermion effective potential. 


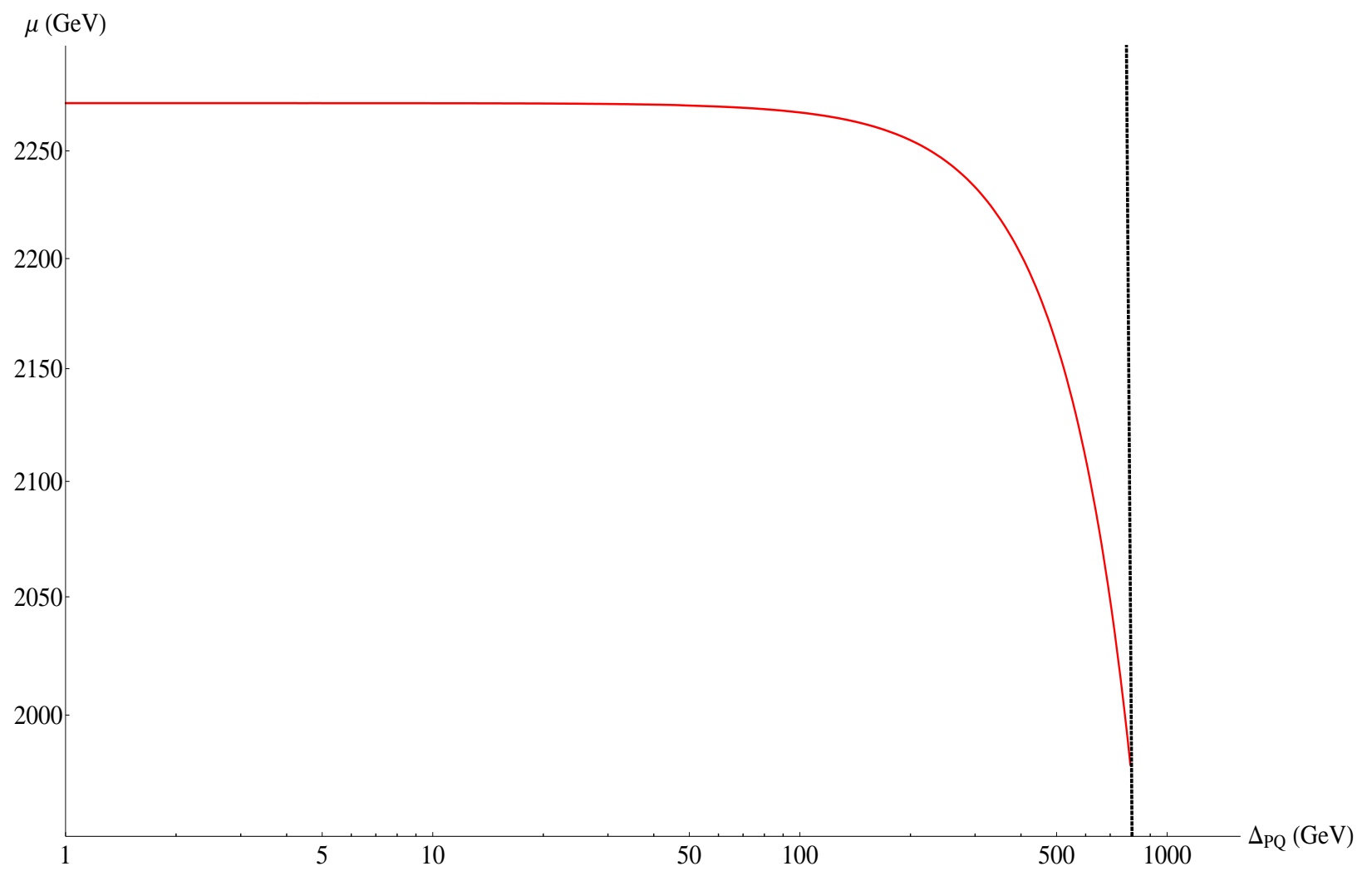

Figure 6: Plot of the parameter $\mu$ as a function of the PQ deformation $\Delta_{P Q}$ in a single messenger model with $\Lambda=5 \times 10^{5} \mathrm{GeV}$. Large values of $\Delta_{P Q}$ produce a tachyon in the effective potential, which appears near the vertical line at $\Delta_{P Q}=10^{2.9} \mathrm{GeV}$.

At such large values of the PQ deformation, we observe that the stau is now the NLSP. In fact, this plot also shows that the masses of the right-handed selectron $\left(\tilde{e}_{R}\right)$ and smuon $\left(\tilde{\mu}_{R}\right)$ also have significantly lower masses, which are nearly comparable to the mass of the bino-like lightest neutralino $\left(\tilde{\chi}^{0}{ }_{1}\right)$. While we certainly expect the scalar masses of the MSSM to receive corrections from the PQ deformation, it is also of interest to note that the Higgs-like charginos $\left(\tilde{\chi}^{ \pm}{ }_{2}\right)$ also decrease in mass. This is primarily due to the fact that the PQ deformation also alters the Higgs potential.

As mentioned above, large values of the PQ deformation decrease the magnitude of the tachyonic mode in the Higgs potential, which alleviates some of the fine-tuning present in the Higgs sector. To a certain extent, this fine-tuning can be quantified as 


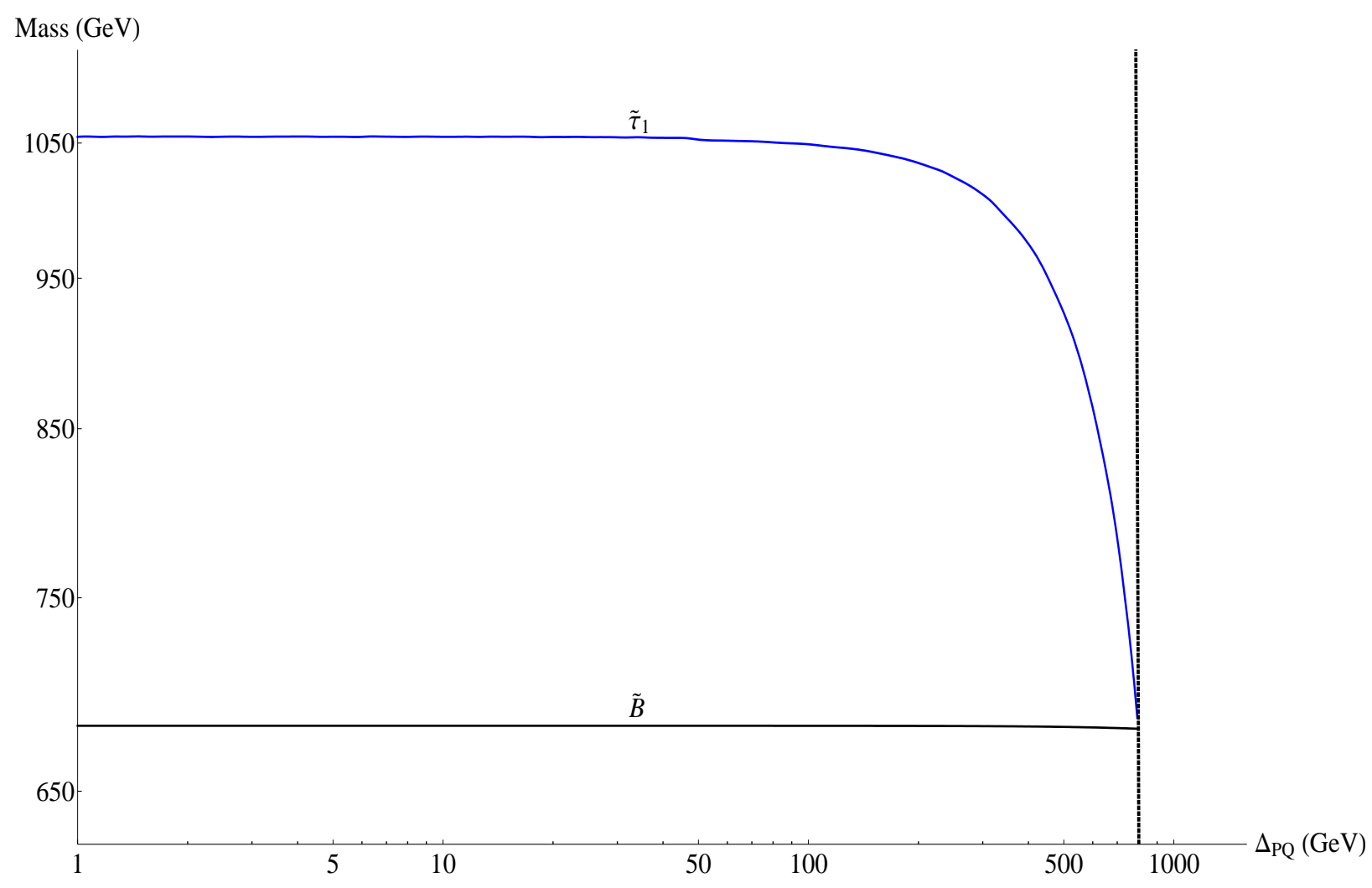

Figure 7: Plot of the stau mass and bino mass as a function of the PQ deformation in a single messenger model with $\Lambda=5 \times 10^{5} \mathrm{GeV}$. Whereas the mass of the bino remains constant, for large values of $\Delta_{P Q}$, the stau mass is comparable in mass to the bino. Near this region, the effective potential develops a tachyonic mode at $\Delta_{P Q}=10^{2.9} \mathrm{GeV}$, which is indicated by the vertical line at the right of the plot. 


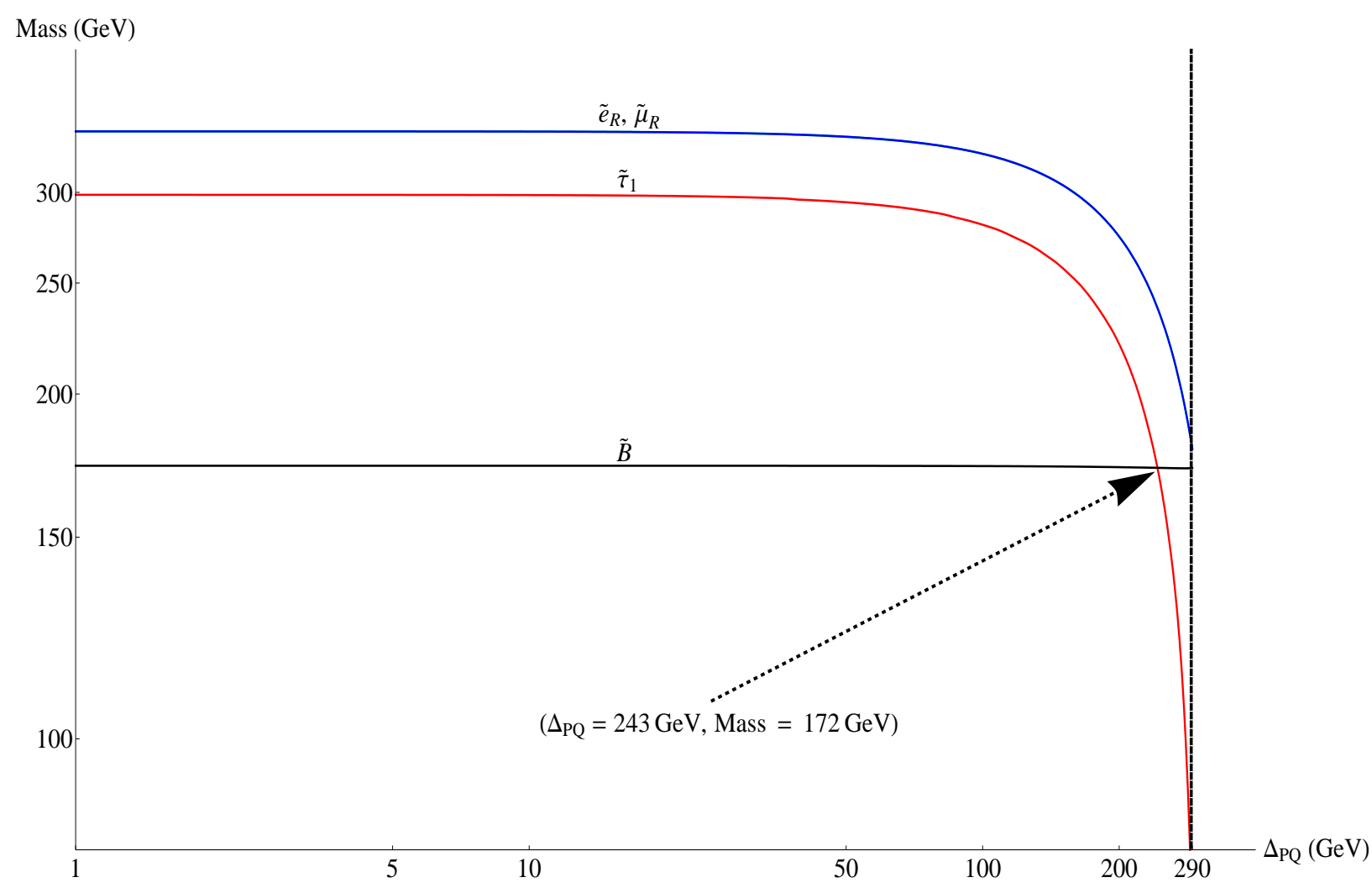

Figure 8: Plot of the stau, smuon, selectron and bino masses as a function of the PQ deformation in a single messenger model with $\Lambda=1.3 \times 10^{5} \mathrm{GeV}$. Whereas the mass of the bino remains constant, for large values of $\Delta_{P Q}$, the other sparticles become lighter, and for a narrow range of values, the stau can in fact become the NLSP. The mass of the stau equals the mass of the bino $(172 \mathrm{GeV})$ at $\Delta_{P Q}=243 \mathrm{GeV}$. Near this region, the effective potential develops a tachyonic mode at $\Delta_{P Q}=290 \mathrm{GeV}$, which is indicated by the vertical line at the right of the plot. 


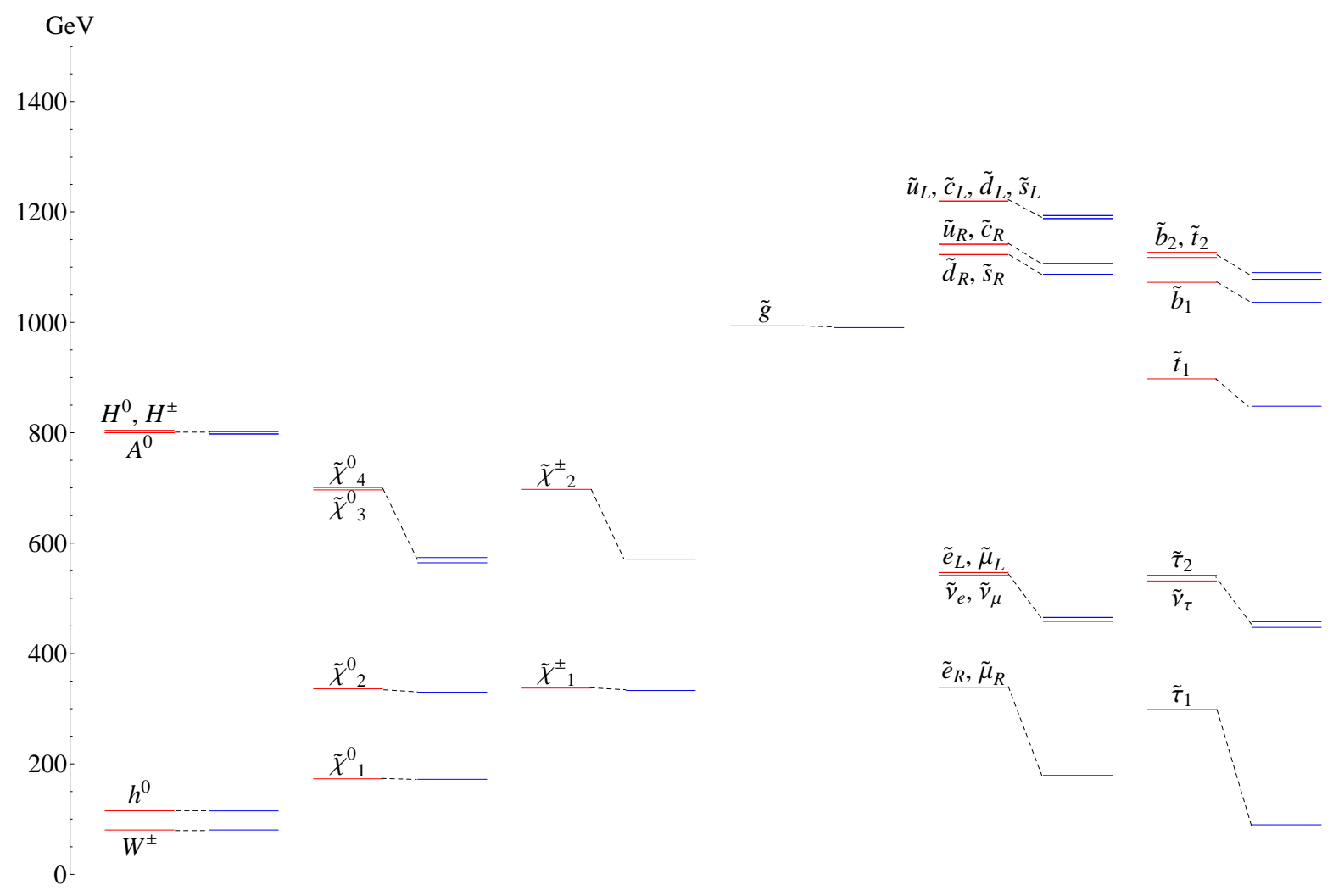

Figure 9: Plot of the sparticle masses separated by pairs of columns in a single messenger model with gaugino mass unification scale $\Lambda=1.3 \times 10^{5} \mathrm{GeV}$ for vanishing PQ deformation (left red columns) and for a deformation of $\Delta_{P Q}=290 \mathrm{GeV}$ (right blue columns). For this particular value of $\Lambda$, larger values of $\Delta_{P Q}$ produce a tachyonic mode in the effective potential. For large deformations, the $\operatorname{stau}\left(\tilde{\tau}_{1}\right)$ is the NLSP. Further, the right-handed selectron $\left(\tilde{e}_{R}\right)$ and smuon $\left(\tilde{\mu}_{R}\right)$ also decrease in mass to the point where they are comparable to the mass of the bino-like lightest neutralino $\left(\tilde{\chi}_{1}^{0}\right)$. Note also that the two Higgs-like neutralinos $\left(\tilde{\chi}_{3}^{0}, \tilde{\chi}^{0}{ }_{4}\right)$ and the Higgs-like charginos $\left(\tilde{\chi}_{2}^{ \pm}\right)$both decrease in mass in the PQ deformed theory. 
in $[72,73]$. Using an existing routine in SOFTSUSY, we have computed the amount of fine-tuning in the mass of the $Z$ boson with respect to $\mu$ using the definition adopted in [72]:

$$
\delta_{\mu}=\left|\frac{\partial \log M_{Z}^{2}}{\partial \log \mu}\right| .
$$

In general, while the PQ deformation appears to decrease the amount of fine-tuning, it does not address in any substantial way the mini-hierarchy problem. This is because at larger values of the PQ deformation, we generically encounter a tachyonic mode in the slepton effective potential which limits the amount of fine-tuning that this deformation can eliminate. For example, with $\Lambda=1.3 \times 10^{5} \mathrm{GeV}$, we find $\delta_{\mu}=2.3 \times 10^{2}$ when $\Delta_{P Q}=0$, while $\delta_{\mu}=1.5 \times 10^{2}$ for $\Delta_{P Q}=290 \mathrm{GeV}$. In this way, the usual problem of fine-tuning at the percent level is present here as well. Nevertheless, with the aim of potentially further reducing the amount of fine-tuning present in this class of models, it would be interesting to determine whether more elaborate F-theory based models could contribute additional soft masses to the Higgs fields, without introducing any effect on the other soft masses of the MSSM. While we do not have an explicit realization of such a scenario, we have also considered the phenomenology of models where the sign of the PQ deformation is reversed so that the soft masses squared of the sleptons and squarks increase while those of the Higgs fields decreases. In this case, the masses of the gauginos effectively remain constant, and the sfermions all increase in mass. This alternate PQ deformation also increases the amount of fine-tuning in the Higgs potential. For example, along this branch, we find that with $\Lambda=1.3 \times 10^{5} \mathrm{GeV}$ and $\Delta_{P Q}=10^{4} \mathrm{GeV}$, the fine-tuning measure $\delta_{\mu}=9.7 \times 10^{4}$, and as is to be expected, the slepton and squarks achieve masses of order $10^{4} \mathrm{GeV}$. For larger values of $\Delta_{P Q}$, a perturbative analysis breaks down. Nevertheless, this at least suggests that for large values of $\Delta_{P Q}$ along this branch, many of the scalar bosons of the spectrum could effectively remain out of present observational reach, but not too far away, perhaps realizing a less extreme version of the split supersymmetry scenario advocated in [74-76].

Having determined detailed features of the sparticle spectrum, it is important to extract potential experimental signatures from this class of models. One immediate avenue of interest would be to determine possible collider signatures at the LHC [77]. Indirect cosmological tests could also serve to constrain the behavior of this class of 
models. As one example, we note that the mass of the gravitino is roughly given by:

$$
m_{3 / 2}=\sqrt{\frac{4 \pi}{3}} \frac{|F|}{M_{p l}} \sim 10^{-2}-10^{-1} \mathrm{GeV}
$$

where $M_{p l} \sim 1.2 \times 10^{19} \mathrm{GeV}$. We find it encouraging that this range of masses for the gravitino appears to be in accord with constraints from big bang nucleosynthesis [78]. In this regard, it is curious to observe that for slightly larger values of the gravitino mass near $1 \mathrm{GeV}$ such as has been advocated in the sweet spot model of supersymmetry breaking [21], there appears to be a slight tension with such constraints. In any case, it would be of great interest to study cosmological constraints on this class of models [79].

\section{Conclusions}

Low scale supersymmetry breaking provides a window into the high energy behavior of local F-theory GUT models. From a bottom up perspective, correlating the scale of supersymmetry breaking with the weak scale imposes additional restrictions on the ultraviolet behavior of the theory. In a broad class of local F-theory models where the Higgs fields and Goldstino chiral superfield $X$ localize on matter curves, correlating these two energy scales translates into the simple geometric condition that these curves must form a triple intersection. In this paper we have shown that $X$ either couples to the Higgs fields through an F-term proportional to $X H_{u} H_{d}$ or through a D-term proportional to $X^{\dagger} H_{u} H_{d}$ which is generated by integrating out the Kalua-Klein modes on the same curve as the zero mode $X$. In the former case, the resulting vev $\langle X\rangle=x+\theta^{2} F$ pushes the masses of the Higgs fields far above the weak scale, exacerbating the $\mu / B \mu$ problem. In the latter case, the resulting D-term realizes a variant of the Giudice-Masiero mechanism. However, the suppression scale for the operator $X^{\dagger} H_{u} H_{d}$ is typically at its largest a few orders of magnitude below the Planck scale. This has the important consequence that in these local models, gravity/moduli mediated supersymmetry breaking generates a $\mu$ term which is far too large. Instead, the scale of supersymmetry breaking must be sufficiently low to solve the $\mu$ problem so that $F \sim 10^{17} \mathrm{GeV}^{2}$. Assuming that the dominant mediation mechanism is instead gauge mediated supersymmetry breaking, bottom 
up considerations also determine the value of $x \sim 10^{12} \mathrm{GeV}$. We have also provided an explicit configuration of intersecting seven-branes which realizes gauge mediated supersymmetry breaking as well as a variant of the Giudice-Masiero mechanism. In this explicit case, all of the fields of the MSSM in addition to the messenger sector are charged under an ambient $U(1)$ Peccei-Quinn gauge symmetry which is typically anomalous. In fact, we have also seen that the phase of $X$ can potentially play the role of the QCD axion. Remarkably, purely bottom up considerations connected to the weak scale automatically determine the axion decay constant to be of order $f_{a}=\sqrt{2}|x| \sim 10^{12} \mathrm{GeV}$, which fits within the available window for invisible axion models. Motivated by these considerations, we next explained why the existence of this $U(1)_{P Q}$ symmetry is particularly natural in many F-theory constructions which contain $E_{6}$ singularities. In addition we have shown that instanton effects in the anomalous $U(1)_{P Q}$ symmetry realized on the worldvolume of a Peccei-Quinn seven-brane can break supersymmetry. The resulting value of $F$ is on the order of $10^{17} \mathrm{GeV}^{2}$ when the scale of the Peccei-Quinn seven-brane is close to the GUT scale. We have also seen that the D-term potential determines $x$ as a function of the minimal amount of flux through the curve supporting $X$, and that this minimal value is $x \sim 10^{12} \mathrm{GeV}$, in beautiful agreement with purely bottom up considerations. Combining all of these elements, we have also characterized the region of MSSM parameter space determined by this class of compactifications. In the remainder of this section we describe some further directions of potential interest.

In this paper we have seen that there is a preferred range of energy scales available for deformed gauge mediated supersymmetry breaking in many local F-theory GUT models. We have also given a precise description of the region of MSSM parameter space defined by these models. It would be of great interest to extract the collider signatures associated with this narrow region of the MSSM parameter space.

We have also shown how higher rank enhancements in the singularity type of F-theory GUT models can make the 'diamond ring model' more natural. Even though we have sketched many elements of this setup, some issues, including how to avoid excess matter fields from additional adjoint representation associated with the higher rank of enhancement remain to be settled in this scenario. It would be important to address these issues in future work.

One of the most elegant features of gravity/moduli mediation models is that the Giudice-Masiero mechanism automatically correlates the scale of supersymmetry 
breaking with the value of the $\mu$ term. On the other hand, we have also seen that in a broad class of models, integrating out the Kaluza-Klein modes of the $X$ field will generate a Giudice-Masiero operator. Insofar as the mass of these heavy modes is below the Planck scale, it is therefore natural to ask whether gauge mediation is always preferred in such cases. Depending on the origin of the $U(1)_{P Q}$ gauge symmetry, there appear to be at least two ways that gravity mediation models could still potentially yield an appropriate value for the $\mu$ term.

When the $U(1)_{P Q}$ gauge symmetry originates from the worldvolume theory of seven-branes, $X$ and the Higgs fields most likely originate from matter curves. In this paper we have assumed that the profile of the zero mode wave function for the $X$ field near a point of triple intersection is an order one number. On the other hand, depending on the choice of signs for gauge fluxes, the local curvature of the del Pezzo surface can repel this gauge singlet wave function away from the point of triple intersection [2]. In this case, the coefficient of the Giudice-Masiero operator could naturally be much smaller, effectively increasing the size of the suppression scale. In this paper we have avoided exploiting this mechanism because it is less predictive, but it is still a viable possibility.

It is also possible that the $U(1)_{P Q}$ gauge symmetry does not originate from the worldvolume theory of a seven-brane. Indeed, the bulk gravity modes of a generic compactification will typically contain several $U(1)$ factors obtained by Kaluza-Klein reduction. In such a scenario, though, it is less clear whether all of the matter content of the visible and messenger sectors possess the correct $U(1)_{P Q}$ charge assignments to allow all required couplings. For these models, the $X$ field may not originate from a matter curve, but could simply be some generic modulus of the compactification. In either case, it would be interesting to study additional properties of such gravity/moduli mediated scenarios.

\section{Acknowledgements}

We thank B.C. Allanach, M. Dine, A.L. Fitzpatrick, G. Giudice, T. Hartman, J. Marsano, N. Saulina, S. Schäfer-Nameki, P. Svrček, A.M. Uranga, H. Verlinde, and E. Witten for helpful discussions. We would also like to thank the Sixth Simons Workshop in Mathematics and Physics for hospitality while some of this work was performed. JJH would also like to thank the 2008 Amsterdam Summer Workshop 
on String Theory for hospitality while some of this work was performed. The work of the authors is supported in part by NSF grant PHY-0244821. The research of JJH was also supported by an NSF Graduate Fellowship.

\section{A Higher Order Instanton Corrections and the Axion Potential}

In section [6] we observed that the phase $a_{x}=\arg x$ directly couples to the QCD instanton density. In order to solve the strong CP problem, the minimum of the effective potential for this field must be sufficiently close to zero. In this regard, the gauged $U(1)_{P Q}$ symmetry shields the axion from many contributions which could a priori have shifted the minimum of its potential. More generally, however, instanton effects could violate this symmetry. In the context of the Fayet-Polonyi model, note that the leading order behavior of the effective potential only depends on the magnitude of $X$, which does not generate a potential for $a_{x}$. But precisely because the experimental bounds on $\theta_{Q C D}$ are so stringent, subleading corrections from instanton effects could also potentially play an important role. Such contributions can either correspond to terms in the superpotential involving just the $X$ fields, or interaction terms between $X$ and fields such as the MSSM Higgs which develop an appropriate vev so that they can contribute to the minimum of the axion potential. In this Appendix we appeal to the $U(1)_{P Q}$ symmetry of the seven-brane theory to characterize the form of higher order instanton corrections, and then estimate their effects on the axion potential. We show that if present, some of these contributions can significantly alter the minimum of the axion potential. But although considerations based on symmetry arguments can constrain the form of possible contributions, they do not establish that such terms are indeed present. To this end, we discuss potential means by which instanton generated contributions to the axion potential can remain in accord with the flatness required to solve the strong CP problem.

\section{A.1 Constraints From Symmetries}

Using symmetry arguments, we now provide a rough characterization of additional instanton generated terms which involve the chiral superfields of the MSSM. Al- 
though the linear term in $X$ in equation (8.21) appears to violate the anomalous $U(1)_{P Q}$ symmetry, the instanton factor $q$ will also transform under this symmetry as well, and axion shifts provide an important constraint on possible contributions [27]. Letting $\alpha$ denote the PQ charge of $q$, because $X$ has PQ charge -4 , equation (8.21) implies:

$$
\alpha=+4
$$

Letting $H$ denote a generic Higgs field, and $\Phi$ any other MSSM chiral superfield, the corresponding PQ charges are respectively -2 and +1 . It follows that a candidate contribution of the form:

$$
W_{\text {inst }}^{\text {tot }} \supset q^{k} H^{a} \Phi^{b} X^{c}
$$

must satisfy the constraint:

$$
4 k-2 a+b-4 c=0
$$

where $a, b, c$ and $k$ are non-negative integers.

\section{A.2 Estimates on Higher Order Corrections}

We now estimate possible higher order instanton corrections to the minimum of the axion potential. The leading order contribution to the axion potential from additional superpotential terms can come from terms quadratic in $X$ such as $X^{2}$ and $H^{2} X^{2}$ so that equation $(\mathrm{A} .3$ reduces to:

$$
\begin{gathered}
X^{2}: k=2 \\
H^{2} X^{2}: k=1 .
\end{gathered}
$$

As shown in detail in [27], there is no contribution to the $k=1$ sector from quadratic

terms in $X$. Expanding to leading order in $q$ therefore yields the X-dependant 
superpotential: 10

$$
W(X)=M_{P Q}^{2} \kappa_{1} \cdot q X+\frac{M_{W}^{2} \kappa_{H}}{2 M_{P Q}} \cdot q X^{2}+\frac{M_{P Q} \kappa_{2}}{2} \cdot q^{2} X^{2}
$$

where $M_{W}$ is shorthand for the mass scale associated with the Higgs vevs, and the $\kappa_{i}$ are moduli dependent worldvolume determinant factors which also include possible contributions from a possibly position dependent axio-dilaton. Assuming that all effects from gravity decouple, the resulting axion potential is:

$$
V_{a x}\left(a_{x}\right)=V_{Q C D}\left(a_{x}\right)+\left|M_{P Q}^{2} \kappa_{1} \cdot q+\frac{M_{W}^{2} \kappa_{H}}{M_{P Q}} \cdot q\right| x\left|e^{i a_{x}}+M_{P Q} \kappa_{2} \cdot q^{2}\right| x\left|e^{i a_{x}}\right|^{2} .
$$

Returning to the bound of line (6.9), the minimum of $V_{a x}$ remains sufficiently close to zero to solve the strong CP problem provided:

$$
\frac{V^{\prime \prime}\left(\theta_{0}\right)}{V_{Q C D}^{\prime \prime}(0)}<10^{-10}
$$

so that:

$$
\begin{gathered}
\frac{\left(M_{P Q}^{2} \kappa_{1} \cdot q\right) \times\left(\frac{M_{W}^{2} \kappa_{H}}{M_{P Q}} \cdot q|x|\right)}{10^{-4} \mathrm{GeV}^{4}}<10^{-10} \\
\frac{\left(M_{P Q}^{2} \kappa_{1} \cdot q\right) \times\left(M_{P Q} \kappa_{2} \cdot q^{2}|x|\right)}{10^{-4} \mathrm{GeV}^{4}}<10^{-10} .
\end{gathered}
$$

This amounts to the two conditions:

$$
\begin{array}{r}
\left|\kappa_{1} \kappa_{H}\right| M_{P Q} M_{W}^{2}|x| \cdot q^{3}<10^{-14} \mathrm{GeV}^{4} \\
\left|\kappa_{1} \kappa_{2}\right| M_{P Q}^{3}|x| \cdot q^{3}<10^{-14} \mathrm{GeV}^{4}
\end{array}
$$

\footnotetext{
${ }^{10}$ In a previous version of this paper, a constant contribution from the instanton sector was assumed to be present. A recent clarification of the analysis appearing in a revised version of [27] illustrates that when an appropriate flux is available such that the Polonyi term is generated, then this constant shift is absent.
} 
Plugging in the explicit values $q \sim 5 \times 10^{-17}, M_{P Q} \sim 4.3 \times 10^{16} \mathrm{GeV},|x| \sim 10^{12}$ $\mathrm{GeV}, M_{W} \sim 10^{2} \mathrm{GeV}$, this requires:

$$
\begin{gathered}
\left|\kappa_{1} \kappa_{H}\right|<2 \times 10^{2} \\
\left|\kappa_{1} \kappa_{2}\right|<10^{-27} .
\end{gathered}
$$

Provided $\kappa_{1}$ and $\kappa_{H}$ are not very large, the contribution from the Higgs dependent contribution will not shift the axion potential by a large amount. The contribution from the purely $X$ sector is far more problematic. We will return to possible means by which the coefficient $\kappa_{2}$ could be arranged to be quite small, so as to satisfy this bound.

On similar grounds, note that there are also instanton contributions to the axion potential from supergravity:

$$
V_{a x} \supset-\frac{3}{M_{p l}^{2}}|W(X)|^{2}=-\frac{3}{M_{p l}^{2}}\left|W_{0}+M_{P Q}^{2} \kappa_{1} \cdot q X\right|^{2},
$$

where here, $W_{0}$ denotes a generic constant shift to the overall superpotential of the theory. The precise value of this constant is tied up with the value of the cosmological constant, and therefore a full discussion of this type of contribution is somewhat beyond the scope of the present paper. Nevertheless, the condition to avoid a significant shift in the axion potential from this contribution is:

$$
\left|W_{0} \kappa_{1}\right| \frac{M_{P Q}^{2}|x|}{M_{p l}^{2}} \cdot q<10^{-14} \mathrm{GeV}^{4}
$$

or:

$$
\left|W_{0} \kappa_{1}\right|<\left(10^{7} \mathrm{GeV}\right)^{3} .
$$

Thus, in order to avoid generating a large contribution to the axion superpotential, we must assume that $W_{0}$ is set by an energy scale smaller than that due to other scales appearing in the gauge mediation sector.

To summarize the discussion above, there is a potentially significant contribution to the axion potential from superpotential terms of the form $q^{2} X^{2}$. In addition, possible constant shifts in the form of the superpotential can also potentially induce corrections to the form of the axion potential. We now proceed to discuss possible 
mechanisms which can rectify this issue.

\section{A.3 Achieving a Flat Axion Potential}

The above analysis establishes that in order for the phase of $X$ to play the role of the QCD axion, several instanton contributions must be sufficiently suppressed. In this subsection we discuss possible resolutions of this issue.

First consider the contribution related to $W_{0}$. As alluded to below line (A.15), the value of $W_{0}$ is also closely connected with the overall value of the cosmological constant. In keeping with the approach espoused in this paper we defer this and other issues related to gravity to a later stage of analysis. Even so, we note that it is conceivable that a fine tuning in $W_{0}$ may be available such that this term is sufficiently small.

Next consider instanton induced contributions of the form $q^{2} X^{2}$. The primary question is whether the coefficient $\kappa_{2}$ can be arranged to be sufficiently small to avoid possible issues with the disruption of the axion potential. There are in principle a few ways in which this type of correction could be arranged, which we now discuss in turn.

One natural possibility is that the characteristic scale multiplying the various instanton contributions might involve a suppression scale closer to $M_{p l}$ rather than $M_{P Q} \sim 4 \times 10^{16} \mathrm{GeV}$. To determine the relevant form of the contribution, note that the higher order instanton contributions can be written as:

$$
W(X)=\kappa_{1} F_{X} \cdot X+\frac{\kappa_{2}}{2} \frac{\left(F_{X} \cdot X\right)^{2}}{M^{3}}+\ldots,
$$

with $F_{X} \sim 10^{17} \mathrm{GeV}^{2}$, and $M$ some suppression scale. Although local considerations naturally suggest the value $M \sim M_{P Q}$, it is in principle possible to also consider larger values such as $M \sim M_{p l} \sim 10^{19} \mathrm{GeV}$. Computing the overall constraint on the flatness of the axion potential, we now obtain the constraint:

$$
\frac{\left|\kappa_{1} \kappa_{2}\right||x| F_{X}^{3} / M_{p l}^{3}}{10^{-4} \mathrm{GeV}^{4}}<10^{-10}
$$

or:

$$
\left|\kappa_{1} \kappa_{2}\right|<10^{-20}
$$


which leads to a slightly less stringent constraint on the $\kappa$ coefficients.

As another possibility, it would be interesting to investigate whether a suitable class of instanton configurations could be found such that only odd powers of $q$ appear. In this case, form of the instanton expansion would be of the form:

$$
W(X)=\kappa_{1} F_{X} \cdot X+\frac{\kappa_{3}}{3} \frac{\left(F_{X} \cdot X\right)^{3}}{M^{6}} \ldots
$$

The overall constraint on the flatness of the axion potential would then be of the form:

$$
\frac{\left|\kappa_{1} \kappa_{3}\right||x|^{2} F_{X}^{4} / M^{6}}{10^{-4} \mathrm{GeV}^{4}}<10^{-10},
$$

so that even for $M \sim 4 \times 10^{16} \mathrm{GeV}$, we obtain:

$$
\left|\kappa_{1} \kappa_{3}\right|<10^{-6}
$$

which again involves less fine tuning in the $\kappa$ 's. For $M \sim M_{p l}$, the constraint is instead:

$$
\left|\kappa_{1} \kappa_{3}\right|<10^{8}
$$

which is a far milder constraint.

In addition to the moduli dependent worldvolume determinant factors, the profile of the axio-dilaton can also lead to an overall suppression of the $\kappa$ 's. Indeed, the zero mode contribution necessary for a bound state of Euclidean D3-branes to contribute often requires the presence of a flux threading the worldvolume of the Euclidean three-brane. Integrating the profile of the axio-dilaton against the instanton density of this flux, the overall position dependence of the axio-dilaton implies that in generall, fluxes with different instanton numbers could produce quite different suppression factors in the $\kappa$ 's.

As a final possibility, it was found in [27] that in order for a Euclidean D3instanton to contribute to the superpotential at all, the flux threading the D3-brane must lift to a trivial class in the threefold base $B_{3}$ of an F-theory compactification. Although beyond the scope of this paper, it would be interesting to determine whether it is possible to arrange for a one-instanton sector to contribute, but to exclude contributions from higher instantons due to a topological obstruction of some kind. 


\section{References}

[1] C. Beasley, J. J. Heckman, and C. Vafa, "GUTs and Exceptional Branes in F-theory - I," arXiv:0802.3391 [hep-th].

[2] C. Beasley, J. J. Heckman, and C. Vafa, "GUTs and Exceptional Branes in F-theory - II: Experimental Predictions," arXiv:0806.0102 [hep-th].

[3] R. Donagi and M. Wijnholt, "Model Building with F-theory," arXiv:0802.2969 [hep-th].

[4] H. Hayashi, R. Tatar, Y. Toda, T. Watari, and M. Yamazaki, "New Aspects of Heterotic-F Theory Duality," arXiv:0805.1057 [hep-th].

[5] L. Aparicio, D. G. Cerdeño, and L. E. Ibáñez, "Modulus-dominated SUSY-breaking soft terms in F-theory and their test at LHC," arXiv:0805.2943 [hep-ph].

[6] J. Marsano, N. Saulina, and S. Schäfer-Nameki, "Gauge Mediation in F-Theory GUT Models," arXiv:0808.1571 [hep-th].

[7] R. Donagi and M. Wijnholt, "Breaking GUT Groups in F-Theory," arXiv:0808.2223 [hep-th].

[8] L. Randall and R. Sundrum, "Out of this world supersymmetry breaking," Nucl. Phys. B557 (1999) 79-118, hep-th/9810155.

[9] G. F. Giudice, M. A. Luty, H. Murayama, and R. Rattazzi, "Gaugino mass without singlets," JHEP 12 (1998) 027, hep-ph/9810442.

[10] R. Dermisek, H. Verlinde, and L.-T. Wang, "Hypercharged Anomaly Mediation," Phys. Rev. Lett. 100 (2008) 131804, arXiv:0711.3211 [hep-ph].

[11] H. Verlinde, L.-T. Wang, M. Wijnholt, and I. Yavin, "A Higher Form (of) Mediation," JHEP 02 (2008) 082, arXiv:0711.3214 [hep-th].

[12] T. W. Grimm and A. Klemm, "U(1) Mediation of Flux Supersymmetry Breaking," arXiv:0805.3361 [hep-th]. 
[13] M. Buican and S. Franco, "SUSY breaking mediation by D-brane instantons," arXiv:0806.1964 [hep-th].

[14] G. F. Giudice and A. Masiero, "A Natural Solution to the $\mu$ Problem in Supergravity Theories," Phys. Lett. B206 (1988) 480-484.

[15] J. P. Conlon, F. Quevedo, and K. Suruliz, "Large-Volume Flux Compactifications: Moduli Spectrum and D3/D7 Soft Supersymmetry Breaking," JHEP 08 (2005) 007, hep-th/0505076.

[16] J. P. Conlon, D. Cremades, and F. Quevedo, "Kähler Potentials of Chiral Matter Fields for Calabi-Yau String Compactifications," JHEP 01 (2007) 022, hep-th/0609180.

[17] J. P. Conlon, "Mirror Mediation," JHEP 03 (2008) 025, arXiv:0710.0873 [hep-th].

[18] J. P. Conlon and F. Quevedo, "Astrophysical and Cosmological Implications of Large Volume String Compactifications," JCAP 0708 (2007) 019, arXiv:0705.3460 [hep-ph].

[19] J. P. Conlon, S. S. Abdussalam, F. Quevedo, and K. Suruliz, "Soft SUSY Breaking Terms for Chiral Matter in IIB String Compactifications," JHEP 01 (2007) 032, hep-th/0610129.

[20] L. J. Hall, J. D. Lykken, and S. Weinberg, "Supergravity as the Messenger of Supersymmetry Breaking," Phys. Rev. D27 (1983) 2359-2378.

[21] M. Ibe and R. Kitano, "Sweet Spot Supersymmetry," JHEP 08 (2007) 016, arXiv:0705.3686 [hep-ph].

[22] Z. Lalak, S. Pokorski, and K. Turzynski, "Gravity in Gauge Mediation," arXiv:0808.0470 [hep-ph].

[23] E. Floratos and C. Kokorelis, "MSSM GUT string vacua, split supersymmetry and fluxes," hep-th/0607217.

[24] M. Cvetič and T. Weigand, "A string theoretic model of gauge mediated supersymmetry breaking," arXiv:0807.3953 [hep-th]. 
[25] J. Kumar, "A Toy Model for Gauge-Mediation in Intersecting Brane Models," arXiv:0808.1264 [hep-th].

[26] H.-C. Cheng and D. E. Kaplan, "Axions and a Gauged Peccei-Quinn Symmetry," hep-ph/0103346.

[27] J. J. Heckman, J. Marsano, N. Saulina, S. Schäfer-Nameki, and C. Vafa, "Instantons and SUSY breaking in F-theory," arXiv:0808.1286 [hep-th].

[28] J. Marsano, N. Saulina, and S. Schäfer-Nameki, "An Instanton Toolbox for F-Theory Model Building," arXiv:0808.2450 [hep-th].

[29] B. C. Allanach, "SOFTSUSY2.0: a program for calculating supersymmetric spectra," Comput. Phys. Commun. 143 (2002) 305-331, hep-ph/0104145.

[30] D. J. H. Chung et. al., "The soft supersymmetry-breaking Lagrangian: Theory and applications," Phys. Rept. 407 (2005) 1-203, hep-ph/0312378.

[31] M. Dine, W. Fischler, and M. Srednicki, "Supersymmetric Technicolor," Nucl. Phys. B189 (1981) 575-593.

[32] S. Dimopoulos and S. Raby, "Supercolor," Nucl. Phys. B192 (1981) 353.

[33] M. Dine and W. Fischler, "A Phenomenological Model of Particle Physics Based on Supersymmetry," Phys. Lett. B110 (1982) 227.

[34] C. R. Nappi and B. A. Ovrut, "Supersymmetric Extension of the $S U(3) \times S U(2) \times U(1)$ Model," Phys. Lett. B113 (1982) 175.

[35] M. Dine and W. Fischler, "A Supersymmetric GUT," Nucl. Phys. B204 (1982) 346.

[36] L. Alvarez-Gaumé, M. Claudson, and M. B. Wise, "Low-Energy Supersymmetry," Nucl. Phys. B207 (1982) 96.

[37] S. Dimopoulos and S. Raby, "Geometric Hierarchy," Nucl. Phys. B219 (1983) 479.

[38] M. Dine and A. E. Nelson, "Dynamical supersymmetry breaking at low-energies," Phys. Rev. D48 (1993) 1277-1287, hep-ph/9303230. 
[39] M. Dine, A. E. Nelson, and Y. Shirman, "Low-energy dynamical supersymmetry breaking simplified," Phys. Rev. D51 (1995) 1362-1370, hep-ph/9408384.

[40] M. Dine, A. E. Nelson, Y. Nir, and Y. Shirman, "New tools for low-energy dynamical supersymmetry breaking," Phys. Rev. D53 (1996) 2658-2669, hep-ph/9507378.

[41] G. F. Giudice and R. Rattazzi, "Theories with Gauge-Mediated Supersymmetry Breaking," Phys. Rept. 322 (1999) 419-499, hep-ph/9801271.

[42] P. Meade, N. Seiberg, and D. Shih, "General Gauge Mediation," arXiv:0801.3278 [hep-ph].

[43] J. Distler and D. Robbins, "General F-Term Gauge Mediation," arXiv:0807.2006 [hep-ph].

[44] N. Arkani-Hamed, M. Dine, and S. P. Martin, "Dynamical supersymmetry breaking in models with a Green-Schwarz mechanism," Phys. Lett. B431 (1998) 329-338, hep-ph/9803432.

[45] M. Y. Khlopov, "Cosmoparticle physics,". Singapore, Singapore: World Scientific (1999) 577 p.

[46] J. E. Kim and G. Carosi, "Axions and the Strong CP Problem," arXiv:0807.3125 [hep-ph].

[47] P. Svrček and E. Witten, "Axions in String Theory," JHEP 0606 (2006) 051, hep-th/0605206.

[48] P. G. Harris et. al., "New Experimental limit on the Electric Dipole Moment of the Neutron," Phys. Rev. Lett. 82 (1999) 904.

[49] M. V. Romalis, W. C. Griffith, and E. N. Fortson, "A new limit on the permanent electric dipole moment of ${ }^{199} \mathrm{Hg}$," Phys. Rev. Lett. 86 (2001) 2505-2508, hep-ex/0012001.

[50] S. Weinberg, "The quantum theory of fields. Vol. 2: Modern applications,". Cambridge, UK: Univ. Pr. (1996) 489 p. 
[51] T. Banks, M. Dine, and M. Graesser, "Supersymmetry, axions and cosmology," Phys. Rev. D68 (2003) 075011, hep-ph/0210256.

[52] P. Fayet, "Weak Interactions of a Light Gravitino: A Lower Limit on the Gravitino Mass from the Decay psi $\rightarrow$ Gravitino anti- Photino," Phys. Lett. B84 (1979) 421.

[53] P. Fayet, "Scattering Cross-Sections of the Photino and the Goldstino (Gravitino) on Matter," Phys. Lett. B86 (1979) 272.

[54] P. Fayet, "Effects of the Spin-1 Partner of the Goldstino (Gravitino) on Neutral Current Phenomenology," Phys. Lett. B95 (1980) 285.

[55] S. M. Barr, "Harmless axions in superstring theories," Phys. Lett. B158 (1985) 397.

[56] S. Katz and C. Vafa, "Matter From Geometry," Nucl. Phys. B 497 (1997) 146-154, hep-th/9606086.

[57] F. Cachazo, K. Intriligator, and C. Vafa, "A Large N Duality via a Geometric Transition," Nucl. Phys. B 603 (2001) 3-41, hep-th/0103067.

[58] F. Cachazo, S. Katz, and C. Vafa, "Geometric Transitions and $\mathcal{N}=1$ Quiver Theories," hep-th/0108120.

[59] R. Bousso and J. Polchinski, "Quantization of four-form fluxes and dynamical neutralization of the cosmological constant," JHEP 06 (2000) 006, hep-th/0004134.

[60] P. Fayet, "Supergauge Invariant Extension of the Higgs Mechanism and a Model for the Electron and its Neutrino," Nucl. Phys. B90 (1975) 104-124.

[61] P. Fayet, "Supersymmetry and Weak, Electromagnetic and Strong Interactions," Phys. Lett. B64 (1976) 159.

[62] P. Fayet, "Spontaneously Broken Supersymmetric Theories of Weak, Electromagnetic and Strong Interactions," Phys. Lett. B69 (1977) 489. 
[63] P. Fayet, "Relations Between the Masses of the Superpartners of Leptons and Quarks, the Goldstino Couplings and the Neutral Currents," Phys. Lett. B84 (1979) 416.

[64] J. Polonyi, "Generalization of the Massive Scalar Multiplet Coupling to the Supergravity," . Hungary Central Inst Res - KFKI-77-93 (77,REC.JUL 78) 5p.

[65] E. Dudas, Y. Mambrini, S. Pokorski, and A. Romagnoni, "Moduli stabilization with Fayet-Iliopoulos uplift," JHEP 04 (2008) 015, arXiv:0711.4934 [hep-th].

[66] R. Blumenhagen, M. Cvetič, and T. Weigand, "Spacetime Instanton Corrections in 4D String Vacua ( - The Seesaw Mechanism for D-Brane Models - )," Nucl. Phys. B771 (2007) 113-142, hep-th/0609191.

[67] L. E. Ibáñez and A. M. Uranga, "Neutrino Majorana Masses From String Theory Instanton Effects," JHEP 03 (2007) 052, hep-th/0609213.

[68] B. Florea, S. Kachru, J. McGreevy, and N. Saulina, "Stringy Instantons and Quiver Gauge Theories," JHEP 05 (2007) 024, hep-th/0610003.

[69] N. J. Craig and D. Green, "Sequestering the Gravitino: Neutralino Dark Matter in Gauge Mediation," arXiv:0808.1097 [hep-ph].

[70] P. Skands et. al., "SUSY Les Houches Accord: Interfacing SUSY Spectrum Calculators, Decay Packages, and Event Generators," JHEP 07 (2004) 036, hep-ph/0311123.

[71] LEP Working Group for Higgs boson searches Collaboration, R. Barate et. al., "Search for the standard model Higgs boson at LEP," Phys. Lett. B565 (2003) 61-75, hep-ex/0306033.

[72] B. de Carlos and J. A. Casas, "One loop analysis of the electroweak breaking in supersymmetric models and the fine tuning problem," Phys. Lett. B309 (1993) 320-328, hep-ph/9303291.

[73] R. Barbieri and A. Strumia, "About the fine-tuning price of LEP," Phys. Lett. B433 (1998) 63-66, hep-ph/9801353. 
[74] J. D. Wells, "Implications of Supersymmetry Breaking with a Little Hierarchy between Gauginos and Scalars," hep-ph/0306127.

[75] N. Arkani-Hamed and S. Dimopoulos, "Supersymmetric Unification Without Low Energy Supersymmetry And Signatures for Fine-Tuning at the LHC," JHEP 06 (2005) 073, hep-th/0405159.

[76] G. F. Giudice and A. Romanino, "Split Supersymmetry," Nucl. Phys. B699 (2004) 65-89, hep-ph/0406088.

[77] "Work in Progress,".

[78] M. Kawasaki, K. Kohri, T. Moroi, and A. Yotsuyanagi, "Big-Bang Nucleosynthesis and Gravitino," arXiv:0804.3745 [hep-ph].

[79] "Work in Progress,". 